Cochrane Database of Systematic Reviews

\title{
Interventions for preventing abuse in the elderly (Review)
}

Baker PRA, Francis DP, Hairi NN, Othman S, Choo WY

Baker PRA, Francis DP, Hairi NN, Othman S, Choo WY.

Interventions for preventing abuse in the elderly.

Cochrane Database of Systematic Reviews 2016, Issue 8. Art. No.: CD010321.

DOI: 10.1002/14651858.CD010321.pub2.

www.cochranelibrary.com 
ABSTRACT 1

PLAIN LANGUAGE SUMMARY

SUMMARY OF FINDINGS

BACKGROUND

Figure 1.

OBJECTIVES

METHODS

Figure 2.

RESULTS

Figure 3.

Figure 4.

DISCUSSION

AUTHORS' CONCLUSIONS

ACKNOWLEDGEMENTS

REFERENCES

CHARACTERISTICS OF STUDIES

ADDITIONAL TABLES

APPENDICES

CONTRIBUTIONS OF AUTHORS

DECLARATIONS OF INTEREST

SOURCES OF SUPPORT

DIFFERENCES BETWEEN PROTOCOL AND REVIEW

INDEX TERMS

\section{TABLE OF CONTENTS}

4

7

9

10

10

14

17

17
19

19

20

24

26

26

28

42

74

85

104

104

104

104

105 
[Intervention Review]

\section{Interventions for preventing abuse in the elderly}

Philip RA Baker ${ }^{1}$, Daniel P Francis ${ }^{1}$, Noran N Hairi2,3, Sajaratulnisah Othman 4 , Wan Yuen Choo 2,3

1School of Public Health and Social Work, Queensland University of Technology, Brisbane, Australia. 2Department of Social \& Preventive Medicine, Faculty of Medicine, University of Malaya, Kuala Lumpur, Malaysia. 3 Julius Centre University of Malaya, University of Malaya, Kuala Lumpur, Malaysia. ${ }^{4}$ Department of Primary Care Medicine, Faculty of Medicine, Kuala Lumpur, Malaysia

Contact: Philip RA Baker, School of Public Health and Social Work, Queensland University of Technology, Brisbane, Queensland, 4059, Australia.p2.baker@qut.edu.au.

Editorial group: Cochrane Public Health Group.

Publication status and date: New, published in Issue 8, 2016.

Citation: Baker PRA, Francis DP, Hairi NN, Othman S, Choo WY. Interventions for preventing abuse in the elderly. Cochrane Database of Systematic Reviews 2016, Issue 8. Art. No.: CD010321. DOI: 10.1002/14651858.CD010321.pub2.

Copyright @ 2016 The Cochrane Collaboration. Published by John Wiley \& Sons, Ltd.

\section{A B S T R A C T}

\section{Background}

Maltreatment of older people (elder abuse) includes psychological, physical, sexual abuse, neglect and financial exploitation. Evidence suggests that $10 \%$ of older adults experience some form of abuse, and only a fraction of cases are actually reported or referred to social services agencies. Elder abuse is associated with significant morbidity and premature mortality. Numerous interventions have been implemented to address the issue of elder maltreatment. It is, however, unclear which interventions best serve to prevent or reduce elder abuse.

\section{Objectives}

The objective of this review was to assess the effectiveness of primary, secondary and tertiary intervention programmes used to reduce or prevent abuse of the elderly in their own home, in organisational or institutional and community settings. The secondary objective was to investigate whether intervention effects are modified by types of abuse, types of participants, setting of intervention, or the cognitive status of older people.

\section{Search methods}

We searched 19 databases (AgeLine, CINAHL, Psycinfo, MEDLINE, Embase, Proquest Central, Social Services Abstracts, ASSIA, Sociological Abstracts, ProQuest Dissertations \& Theses Global, Web of Science, LILACS, EPPI, InfoBase, CENTRAL, HMIC, Opengrey and Zetoc) on 12 platforms, including multidisciplinary disciplines covering medical, health, social sciences, social services, legal, finance and education. We also browsed related organisational websites, contacted authors of relevant articles and checked reference lists. Searches of databases were conducted between 30 August 2015 and 16 March 2016 and were not restricted by language.

\section{Selection criteria}

We included randomised controlled trials (RCTs), cluster-randomised trials, and quasi-RCTs, before-and-after studies, and interrupted time series. Only studies with at least 12 weeks of follow-up investigating the effect of interventions in preventing or reducing abuse of elderly people and those who interact with the elderly were included.

\section{Data collection and analysis}

Two review authors independently extracted data and assessed the studies' risk of bias. Studies were categorised as: 1) education on elder abuse, 2) programmes to reduce factors influencing elder abuse, 3) specific policies for elder abuse, 4) legislation on elder abuse, 5) programmes to increase detection rate on elder abuse, 6) programmes targeted to victims of elder abuse, and 7) rehabilitation programmes for perpetrators of elder abuse. All studies were assessed for study methodology, intervention type, setting, targeted audience, intervention components and intervention intensity. 


\section{Main results}

The search and selection process produced seven eligible studies which included a total of 1924 elderly participants and 740 other people. Four of the above seven categories of interventions were evaluated by included studies that varied in study design. Eligible studies of rehabilitation programmes, specific policies for elder abuse and legislation on elder abuse were not found. All included studies contained a control group, with five of the seven studies describing the method of allocation as randomised. We used the Cochrane 'Risk of bias' tool and EPOC assessment criteria to assess risk of bias. The results suggest that risk of bias across the included body of research was high, with at least $40 \%$ of the included studies judged as being at high risk of bias. Only one study was judged as having no domains at high risk of bias, with two studies having two of 11 domains at high risk. One study was judged as being at high risk of bias across eight of 11 domains.

All included studies were set in high-income countries, as determined by the World Bank economic classification (USA four, Taiwan one, UK two). None of the studies provided specific information or analysis on equity considerations, including by socio-economic disadvantage, although one study was described as being set in a housing project. One study performed some form of cost-effectiveness analysis on the implementation of their intervention programmes, although there were few details on the components and analysis of the costing.

We are uncertain whether these interventions reduce the occurrence or recurrence of elder abuse due to variation in settings, measures and effects reported in the included studies, some of which were very small and at a high risk of bias (low-and very low-quality evidence).Two studies measured the occurrence of elder abuse. A high risk of bias study found a difference in the post-test scores ( $P$ value 0.048 and 0.18 ). In a low risk of bias study there was no difference found (adjusted odds ratio $(O R)=0.48,95 \% 0.18$ to 1.27$)(n=214)$. For interventions measuring abuse recurrence, one small study $(n=16)$ reported no difference in post-test means, whilst another found higher levels of abuse reported for the intervention arms (Cox regression, combined intervention hazard ratio $(H R)=1.78$, alpha level $=0.01$ ).

It is uncertain whether targeted educational interventions improve the relevant knowledge of health professionals and caregivers (very low-quality evidence), although they may improve detection of resident-to-resident abuse. The concept of measuring improvement in detection or reporting as opposed to measuring the occurrence or recurrence of abuse is complicated. An intervention of public education and support services aimed at victims may also improve rates of reporting, however it is unclear whether this was due to an increase in abuse recurrence or better reporting of abuse.The effectiveness of service planning interventions at improving the assessment and documentation of related domains is uncertain. Unintended outcomes were not reported in the studies.

\section{Authors' conclusions}

There is inadequate trustworthy evidence to assess the effects of elder abuse interventions on occurrence or recurrence of abuse, although there is some evidence to suggest it may change the combined measure of anxiety and depression of caregivers. There is a need for high-quality trials, including from low- or middle-income countries, with adequate statistical power and appropriate study characteristics to determine whether specific intervention programmes, and which components of these programmes, are effective in preventing or reducing abuse episodes among the elderly. It is uncertain whether the use of educational interventions improves knowledge and attitude of caregivers, and whether such programmes also reduce occurrence of abuse, thus future research is warranted. In addition, all future research should include a component of cost-effectiveness analysis, implementation assessment and equity considerations of the specific interventions under review.

\section{PLAIN LANGUAGE SUMMARY}

\section{Interventions for preventing abuse of older people}

\section{Review question}

Our aim was to identify if specific programs or strategies are useful to prevent or reduce abuse in older people (60 years and over). We looked to include studies that described the effect of these programs or strategies whether aimed at the elderly themselves or people (such as caregivers or nursing home staff) with whom they interact.

\section{Background}

Elder abuse - physical, psychological, sexual abuse, neglect and/or financial exploitation - is common but often underreported. Elder abuse can be a single, or repeated act, or may be a lack of appropriate action. Elder abuse occurs within a relationship where there is an expectation of trust, but regrettably harm or distress is caused to the older person. The abuse can often come from someone who they know well or have relationship with such as a spouse, partner, family member, or friend. It can also be caused by service providers in institutions and healthcare settings. It is most likely to occur when staff have inadequate training and supervision, or lack sufficient resources to undertake their responsibilities. This is a global problem that affects millions of older people resulting in great economic costs to both the individuals and the healthcare system. Abuse can lead to poorer health, injuries and even premature death.

\section{Search date}

All databases were searched up to August 2015. Additional searches of the main databases were conducted between 30 August 2015 and 16 March 2016.

\section{Study characteristics}


We found seven studies from our search of 19 databases. All together, the studies included 1924 elderly participants and 740 people (such as carers or nursing home staff) with whom they interact. These studies described methods of preventing or reducing elder abuse with elderly people. The studies included programs and strategies that took place in many different settings (home, community, institutions) although only in high-income countries. The programs and strategies identified included methods to increase detection in clinical practice and community settings, victim support, increasing awareness of elder abuse and delivering training programs aimed at building skills in care providers. Most studies described changes in knowledge and attitudes, with very few measuring the occurrence or reoccurrence of abuse. The study durations ranged from six to 24 months.

\section{Key results}

The included studies suggest it is uncertain whether targeted educational interventions improve the knowledge of health and allied professionals and caregivers about elder abuse. It is unclear whether any improved knowledge actually leads to changes in the way they behave thereafter, and whether this leads to the elderly being abused less. Similarily, supporting and educating elderly victims of abuse appears to lead to more reporting of abuse, however it is unclear if the higher reporting meant more abuse occurred or a greater willingness to report the abuse as it occurred.

None of the studies reported any unintended outcomes of these approaches.

\section{Quality of the evidence}

Most of the evidence was low or very low quality (we cannot assume the findings of these studies are true) and limits our understanding of what strategies or programs work best to decrease or prevent elder abuse. Many of the studies were unclear in the design, too small in size or not similar enough in their findings to have full confidence in the findings. 


\section{SUMMARY OF FINDINGS}

\section{Summary of findings for the main comparison.}

\section{Educational interventions compared with control for preventing elderly abuse}

Patient or population: Carers of elderly persons

Settings: Workforce training

\section{Intervention: Educational interventions}

Comparison: Control - no specific training

\begin{tabular}{|c|c|c|c|c|}
\hline $\begin{array}{l}\text { Outcomes (duration of fol- } \\
\text { low-up) }\end{array}$ & Summary of effects & $\begin{array}{l}\text { No of Partici- } \\
\text { pants } \\
\text { (studies) }\end{array}$ & $\begin{array}{l}\text { Quality of the } \\
\text { evidence } \\
\text { (GRADE) }\end{array}$ & Comments \\
\hline $\begin{array}{l}\text { Occurrence of abuse - care- } \\
\text { givers: Caregiver's abusive be- } \\
\text { haviours (Caregiver Psycho- } \\
\text { logical Elder Abuse Behaviour } \\
\text { Scale) (duration not specified, } \\
\text { assumed } 10 \text { months) }\end{array}$ & $\begin{array}{l}\text { Abusive behaviour typically lower in } \\
\text { the trained caregiver group (e.g. ad- } \\
\text { justed mean difference }-3.46 \text {, adjust- } \\
\text { ed } \% \text { change } 11.4 \% \text { ) }\end{array}$ & $\begin{array}{l}112 \text { caregivers } \\
\text { (1 study) }\end{array}$ & $\oplus \circ \circ \circ$ Very Low ${ }^{1}$ & $\begin{array}{l}\text { One study in- } \\
\text { cluded this pri- } \\
\text { mary outcome }\end{array}$ \\
\hline $\begin{array}{l}\text { Occurrence of abuse by el- } \\
\text { derly persons: Detecting resi- } \\
\text { dent-to-resident abuse ( } 6 \& 12 \\
\text { months) }\end{array}$ & $\begin{array}{l}\text { Intervention group reported more in- } \\
\text { cidents at } 6 \& 12 \text { months for the in- } \\
\text { tervention than the control (adjust- } \\
\text { ed mean difference to the control of } \\
420 \%) \# 1\end{array}$ & $\begin{array}{l}325 \text { caregiver } \\
\text { nurses, } 1405 \text { res- } \\
\text { idents } \\
\text { (1 study) }\end{array}$ & $\oplus \oplus \circ \circ$ Low $^{2}$ & $\begin{array}{l}\text { One study in- } \\
\text { cluded this pri- } \\
\text { mary outcome }\end{array}$ \\
\hline $\begin{array}{l}\text { Knowledge and attitude to el- } \\
\text { der abuse ( } 6 \text { to } 12 \text { months) }\end{array}$ & $\begin{array}{l}\text { Knowledge generally improved after } \\
\text { intervention (e.g. KAMA scores adjust- } \\
\text { ed mean change } 25.8 \% \text {, KGNS 5\%) }\end{array}$ & $\begin{array}{l}523 \text { caregivers } \\
\text { ( } 3 \text { studies) }\end{array}$ & $\oplus \circ \bigcirc^{3}$ Very Low & $\begin{array}{l}\text { Substantial het- } \\
\text { erogeneity be- } \\
\text { tween trials re- } \\
\text { garding type of } \\
\text { interventions } \\
\text { and measured } \\
\text { outcomes. }\end{array}$ \\
\hline
\end{tabular}

GRADE Working Group grades of evidence

High quality: Further research is very unlikely to change our confidence in the estimate of effect.

Moderate quality: Further research is likely to have an important impact on our confidence in the estimate of effect and may change the estimate.

Low quality: Further research is very likely to have an important impact on our confidence in the estimate of effect and is likely to change the estimate.

Very low quality: We are very uncertain about the estimate.

1 Downgraded three levels for non-randomised study, serious risk of bias and imprecision

2 Downgraded two levels for serious risk of bias and possible contamination

3 Downgraded three levels for substantial heterogeneity and risk of bias in the 3 studies, and the inclusion of one non-RCTstudy of 112 caregivers as 'very low',

\#1 Refers to 12-month result

\section{Summary of findings 2 .}

\section{Programs to reduce factors influencing elderly abuse}

\section{Patient or population: Carers of elderly persons}


Settings: Caregivers of family members suffering from dementia

Intervention: Reducing factors influencing elderly abuse through promoting the mental health of caregivers

\begin{tabular}{|c|c|c|c|c|}
\hline $\begin{array}{l}\text { Outcomes (duration of fol- } \\
\text { low-up) }\end{array}$ & Summary of effects & $\begin{array}{l}\text { No of Partici- } \\
\text { pants } \\
\text { (studies) }\end{array}$ & $\begin{array}{l}\text { Quality of the } \\
\text { evidence } \\
\text { (GRADE) }\end{array}$ & Comments \\
\hline $\begin{array}{l}\text { Occurrence of abuse - care- } \\
\text { givers: Caregiver's abusive } \\
\text { behaviours (Modified conflict } \\
\text { tactics scale) ( } 8 \text { months) }\end{array}$ & $\begin{array}{l}\text { No statistical difference in abusive } \\
\text { behaviour between the groups(ad- } \\
\text { justed OR } 0.48,95 \% \mathrm{Cl} 0.18 \text { to } 1.27 \text { ) }\end{array}$ & $\begin{array}{l}260 \text { caregivers } \\
\text { ( } 1 \text { study) }\end{array}$ & $\oplus \oplus \circ \circ$ Low $^{1}$ & $\begin{array}{l}\text { One low risk of bias } \\
\text { study which ap- } \\
\text { peared inadequate- } \\
\text { ly powered includ- } \\
\text { ed this primary out- } \\
\text { come }\end{array}$ \\
\hline $\begin{array}{l}\text { Anxiety and depression: to- } \\
\text { tal scores on hospital anxiety } \\
\text { and depression scale (HADS) } \\
\text { (8 months) }\end{array}$ & $\begin{array}{l}\text { Mean total HADS score lower for } \\
\text { the intervention group of care- } \\
\text { givers than the control }(-1.80 \\
\text { points }(95 \% \mathrm{Cl}-3.29 \text { to }-0.31, \mathrm{P}= \\
0.02)\end{array}$ & $\begin{array}{l}260 \text { caregivers } \\
\text { (1 study) }\end{array}$ & $\oplus \oplus \oplus \circ$ Moderate 2 & $\begin{array}{l}\text { One low risk of bias } \\
\text { study included this } \\
\text { secondary outcome }\end{array}$ \\
\hline
\end{tabular}

GRADE Working Group grades of evidence

High quality: Further research is very unlikely to change our confidence in the estimate of effect.

Moderate quality: Further research is likely to have an important impact on our confidence in the estimate of effect and may change the estimate.

Low quality: Further research is very likely to have an important impact on our confidence in the estimate of effect and is likely to change the estimate.

Very low quality: We are very uncertain about the estimate.

1 Downgraded two level for very serious imprecision

2 Downgraded one level based on only one study reporting as a secondary outcome

\section{Summary of findings 3.}

\section{Programs for increasing detection for preventing elderly abuse}

Patient or population: Carers of elderly persons with a responsibility for detecting abuse

Settings: Community mental organisations and home care organisations

Intervention: Programs for increasing detection for preventing elderly abuse through the provision of a toolkit

\begin{tabular}{|c|c|c|c|c|}
\hline $\begin{array}{l}\text { Outcomes (du- } \\
\text { ration of fol- } \\
\text { low-up) }\end{array}$ & Summary of effects & $\begin{array}{l}\text { No of Partici- } \\
\text { pants } \\
\text { (studies) }\end{array}$ & $\begin{array}{l}\text { Quality of the } \\
\text { evidence } \\
\text { (GRADE) }\end{array}$ & Comments \\
\hline $\begin{array}{l}\text { Occurrence of el- } \\
\text { der abuse }\end{array}$ & Outcome was not reported for this comparison & & & \\
\hline $\begin{array}{l}\text { Clinician assess- } \\
\text { ment practices ( } 1 \\
\text { year) }\end{array}$ & $\begin{array}{l}\text { The study authors claimed that there was a signifi- } \\
\text { cant increase in the proportion of clinicians in con- } \\
\text { ducting clinical assessments in neglect and abuse } \\
\text { domain, however they failed to provide statistical } \\
\text { analyses to support this conclusion }\end{array}$ & $\begin{array}{l}13 \text { agencies, } 44 \\
\text { clinicians, } 100 \text { el- } \\
\text { derly persons } \\
\text { (1 study) }\end{array}$ & $\oplus \circ \circ \circ$ Very low 1 & $\begin{array}{l}\text { One high risk of } \\
\text { bias study in- } \\
\text { cluded this sec- } \\
\text { ondary outcome }\end{array}$ \\
\hline
\end{tabular}

GRADE Working Group grades of evidence

High quality: Further research is very unlikely to change our confidence in the estimate of effect.

Moderate quality: Further research is likely to have an important impact on our confidence in the estimate of effect and may change the estimate. 
Low quality: Further research is very likely to have an important impact on our confidence in the estimate of effect and is likely to change the estimate.

Very low quality: We are very uncertain about the estimate.

1 Downgraded three levels based on only one non-randomised study with very serious risks of bias and a lack of transparency in the analysis of this secondary outcome (indirectness)

\section{Summary of findings 4.}

Programs targeted to victims for preventing elderly abuse

Patient or population: Victims of abuse

Settings: Community settings

Intervention: Programs targeted to victims for preventing elderly abuse including the provisions of psycho-education support and materials

\begin{tabular}{|c|c|c|c|c|}
\hline Outcomes (duration of follow-up) & $\begin{array}{l}\text { Summary of ef- } \\
\text { fects }\end{array}$ & $\begin{array}{l}\text { No of Partici- } \\
\text { pants } \\
\text { (studies) }\end{array}$ & $\begin{array}{l}\text { Quality of the } \\
\text { evidence } \\
\text { (GRADE) }\end{array}$ & Comments \\
\hline $\begin{array}{l}\text { Recurrence of abuse: Physical abuse } \\
\text { - Hartford study physical abuse sub- } \\
\text { scale ( } 8 \text { weeks post intervention) }\end{array}$ & $\begin{array}{l}\text { Unable to deter- } \\
\text { mine }\end{array}$ & $16(1 \text { study })^{3}$ & $\oplus \circ \circ \circ$ Very low 1 & $\begin{array}{l}\text { One very small study at high risk } \\
\text { of bias included this primary } \\
\text { outcome }\end{array}$ \\
\hline $\begin{array}{l}\text { Recurrence of abuse - elderly per- } \\
\text { sons: Modified version of the Con- } \\
\text { flict Tactic Scale ( } 6 \& 12 \text { months) }\end{array}$ & $\begin{array}{l}\text { Higher reports of } \\
\text { victimisation }\end{array}$ & 403 (1 study) & $\oplus \oplus \circ \circ$ Low $^{2}$ & $\begin{array}{l}\text { It is unclear whether this in- } \\
\text { crease reflects an increase in } \\
\text { the rate of abuse recurrence } \\
\text { (more abuse) or better report- } \\
\text { ing of abuse. }\end{array}$ \\
\hline
\end{tabular}

GRADE Working Group grades of evidence

High quality: Further research is very unlikely to change our confidence in the estimate of effect.

Moderate quality: Further research is likely to have an important impact on our confidence in the estimate of effect and may change the estimate.

Low quality: Further research is very likely to have an important impact on our confidence in the estimate of effect and is likely to change the estimate.

Very low quality: We are very uncertain about the estimate.

1 Downgraded three levels for very serious risk of bias, very sparse data from one study.and a resulting in lack of clarity

2 Downgraded two levels for very serious risk of bias.

3 This number includes one participant unaccounted for in the analysis. The data analysis presented by the authors includes 15 individuals. 


\section{B A C K G R O U N D}

\section{Description of the condition}

Maltreatment of older people (or commonly termed as elder abuse) is a global problem, affecting millions of older people worldwide. It was recently reported that elder abuse was responsible for 2500 deaths a year in Europe (WHO 2011). These figures will inevitably elevate with populations ageing and living longer. According to the World Health Organization (WHO), elder abuse is defined as "a single, or repeated act, or lack of appropriate action, occurring within any relationship where there is an expectation of trust, which causes harm or distress to an older person" (WHO 2002a). This definition encompasses harms towards the elderly by people they know or with whom they have a relationship, such as a spouse, partner or family member, a friend or neighbour, or those they rely on for services (Action on Elder Abuse 1995a). The research literature in this area tends to refer to elder abuse as physical abuse, psychological or emotional abuse, financial abuse (or financial exploitation), sexual abuse, and neglect (Cooper 2008; Lachs 2004).

Routine data on elder abuse remain scarce and have a short history with first references to "granny bashing" appearing in the literature in 1975 (Baker 1975; Burston 1975). The exact scale of the problem has been difficult to determine, given the varying definitions and social norms across the world (Kosberg 2003). Available community-based studies contain evidence that abuse, neglect and financial exploitation of elders are much more a universal phenomenon than societies admit. A review of the prevalence of elder abuse found the overall elder abuse rate ranged between $3.2 \%$ and $27.5 \%$, with significantly higher rates among vulnerable older people (Cooper 2008). Data suggest that $2.7 \%$ of elderly people reported physical maltreatment, $19.4 \%$ reported mental abuse, $0.7 \%$ reported sexual abuse and 3.8\% reported financial abuse in the previous year (Soares 2010). The prevalence of maltreatment is reported to be much higher among vulnerable dependent elderly requiring care, particularly those in nursing and residential homes. In a survey in the United States, $24.3 \%$ of elderly relatives reported at least one incident of physical abuse by staff in nursing homes in the previous 12 months (Schiamberg 2012). In rural China, more than one-third of elderly people reported elder abuse, with psychological mistreatment and caregiver neglect being the most common types of abuse (Wu 2012). Elderly people with dementia are also reported to be at a higher risk of being abused by family carers (Cooper 2009).

Emerging evidence shows that elder maltreatment has great economic costs, including the direct costs to health, social, legal, police and other services. The direct cost arising from maltreatment is attributed to increased healthcare costs to treat and rehabilitate the maltreated elderly. It was estimated that the direct healthcare costs of injuries due to elder maltreatment has contributed more than USD 5.3 billion to the annual healthcare expenditure in the United States (Mouton 2004). Maltreated elderly were found to have longer hospital stays and higher rates of utilisation of emergency services compared to their non-maltreated counterparts (Dong 2012; Dong 2013). In Australia, costs due to hospital admissions for elder maltreatment were estimated to be between AUD 9.9 million and AUD 30.7 million for 2007/2008 (Jackson 2009). Other costs include provision of protection and care by the legal and social system, such as adult social services agencies, which spent at least USD 500 million in 2004 alone (Dyer 2007; NCEA 2006). In addition, financial abuse could seriously affect older people who survive on limited resources. Indirect costs as a consequence of elder maltreatment include loss of productivity of caregivers, inability to continue with activities of daily life, diminished quality of life and lost investment in social capital (Butchart 2008). Given these enormous social and economic costs, there is a dire need for evidence-based and immediate actions on elder abuse.

Elder abuse is a result of complex interactions among factors at the individual, relationship, community and societal levels, which can be conceptualised using an ecological perspective (Gordon 2001; Wolf 2003). Factors from each level can interact, putting the elderly at risk of abuse. For example, older people with dementia (Dyer 2000; Hansberry 2005), disabilities (Ansello 2010; Laumann 2008) and chronic health problems (Lowenstein 2009) that result in increased dependence on caregivers are particularly at risk of elder maltreatment. Furthermore, low social support, loneliness, social isolation and lack of social networks among the elderly further perpetuate maltreatment (Acierno 2010; Dong 2007; Dong 2009). Perpetrators' mental illness, high levels of hostility, substance abuse, psychological distress and their dependence on the victim for accommodation and financial support appear to be strong risk factors that predispose elderly to maltreatment (Jackson 2011; Schiamberg 2000). Women were generally significantly more likely to have experienced maltreatment than men, but this may differ according to the type of abuse (Biggs 2009). Intergenerational transmission of violent behaviour is a plausible risk factor, given the association found between history of childhood violence with child abuse and other forms of aggressive behaviour (Biggs 2009; Jackson 2011; Lowenstein 2009; Yan 2003). Distinctive characteristics were found to be associated with greater maltreatment in institutions and healthcare settings (or institutional abuse) and include inadequate staff training and supervision, inadequate staff to carry out daily activities, and prejudiced attitudes towards elderly (Jogerst 2008a; Phillips 2011).

Community factors that exacerbate elder maltreatment include high crime rates, social disorganisation, lack of social resources and networks, and poverty (Luo 2011). Further, societal-level factors that have been connected with elder abuse include culture, ethnicity and policies. These are evident in the different views on what constitutes elder abuse as well as the societal response to the problems that exist between different ethnic and cultural groups and can influence the reporting of the problem to health and protection services (Dakin 2009; Moon 1993; Wolf 1999). In addition, customary practices in some societies might be judged to be abusive in some countries but not in others (Kosberg 2003; Podnieks 2010).

\section{Description of the intervention}

This review encompasses any strategy or intervention that could be utilised to prevent or reduce elder abuse. Our definition encompasses interventions that have been developed to address the multifaceted nature of elder abuse, targeted at different levels reflecting the socio-ecological approach, that is, at individual, familial, community and societal levels. Browne and Herbert (1997) identified three fundamental types of interventions that could operate at different levels. Based on their classification, we define primary prevention as interventions related to preventing the abuse from occurring, secondary intervention as actions aimed to prevent further abuse, and tertiary intervention as actions to manage the consequences after abuse has occurred (Browne 1997). 
We included primary prevention activities that could be individually-focused activities, community-based interventions or changes in policies. Individually-focused activities could involve interventions targeting the elderly directly, their family members, or both. For example, health educational and skills-based programmes have been specifically developed for the elderly and their families to provide them with the skills to communicate effectively, manage stress, resolve conflicts, and promote healthier relationships (Hsieh 2009). Other approaches are those which encourage positive attitudes towards older people by increasing meaningful interaction between elderly and younger persons via an intergenerational programme. Other programmes target schools, university students or youths from community settings, such as church groups and employment programmes (Fujiwara 2006; Hermoso 2006; Sanders 2008), and may vary in the type of activities conducted. Within this, activities could range from simply exchanging letters or e-mails to long-term or direct engagement with the elderly through participation in joint community projects, group activities, or help with activities of daily living (e.g. gardening, house cleaning or tutoring).

Community-based interventions such as awareness campaigns and health education conducted across society using mass media such as television, radio, printed materials and Internet web sites will be included in this review. Such campaigns are generally designed and implemented to raise awareness of elder maltreatment, encourage respectful and dignified treatment of older people, and provide education about available support services that, in turn, may prevent elderly abuse (HSE 2009).

It cannot be assumed that an intervention programme being implemented will bring only beneficial effects. Some interventions might endanger elders due to inappropriate risk assessment, breach of confidentiality, invasion of privacy and failure in safety plan (Dugan 2003). For example, several studies suggest that interventions such as psychologically-based programmes (e.g. anger, stress and coping management) (Cooper 2015), behavioural therapy (Drossel 2011), provision of respite care or temporary relief care (Jeon 2005), and social support groups (Brownell 2006) for family members or caregivers may reduce risk factors of elder abuse, such as caregivers' stress and dependency of elderly. However, several evaluations have reported an increase in maltreatment following interventions such as the restraints reduction programme (mechanical devices or barriers that restrict the movement of a person in a chair, wheelchair, or bed), homevisiting programme, and advocate volunteers (Davis 2001; Filinson 1993). Therefore, we also considered the negative consequences associated with the elder abuse prevention strategies in this review.

\section{How the intervention might work}

This review aims to give a broader perspective on the interventions to prevent or reduce elder abuse. Elder abuse interventions occur in a range of settings, including healthcare and social or legal settings, and they may be primary, secondary and tertiary in nature.

A logic model was developed to capture the broad range of approaches that may be used to prevent or reduce elder abuse (Figure 1). It also articulates a range of possible short- and longterm outcomes that may be used to measure the effectiveness of interventions and capture the levels where the intervention may be operating. Short-term outcomes include participant-, victim- or perpetrator-related outcomes, such as increased knowledge, attitudes and skills, identification of abuse and elderly independent living. Long-term outcomes could include lower rates of elder abuse reporting or a reduction in the recurrence of elder abuse. 
Figure 1. Footnotes (Figure 1): 1Programmes to reduce factors influencing elder abuse (e.g. respite care, social support, psychological programme, restraint reduction, intergenerational programme) ${ }^{2}$ Legislation (e.g. advocacy-based programme, law-orientated programme, legal institution, elderly act, mandatory reporting, adult protection statutes) ${ }^{3}$ Specific policies for elderly (e.g. improve housing, transport, aged friendly cities, banking, pension, welfare aid) 4 Programmes to increase detection rate for prevention (e.g. home visit, home-based geriatric assessment, helpline, training for healthcare workers and social worker, guideline/ protocol, screening) 5 Programmes targeted to victims (e.g. adult protective services, emergency shelter, temporary residential services) ${ }^{6}$ Rehabilitation programmes (e.g. legal assistance, psychiatric intervention, support, counselling) 70 ther professionals (e.g. legislators, policy makers, politicians, journalists) 8 Increase identification (e.g. increase detection rate, increase reporting)

Figure 1. Interventions for preventing elderly abuse

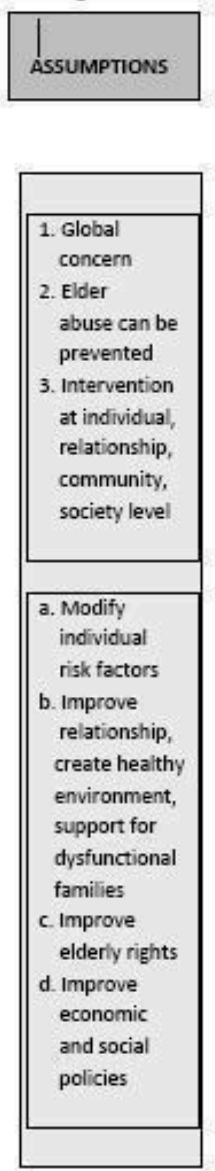
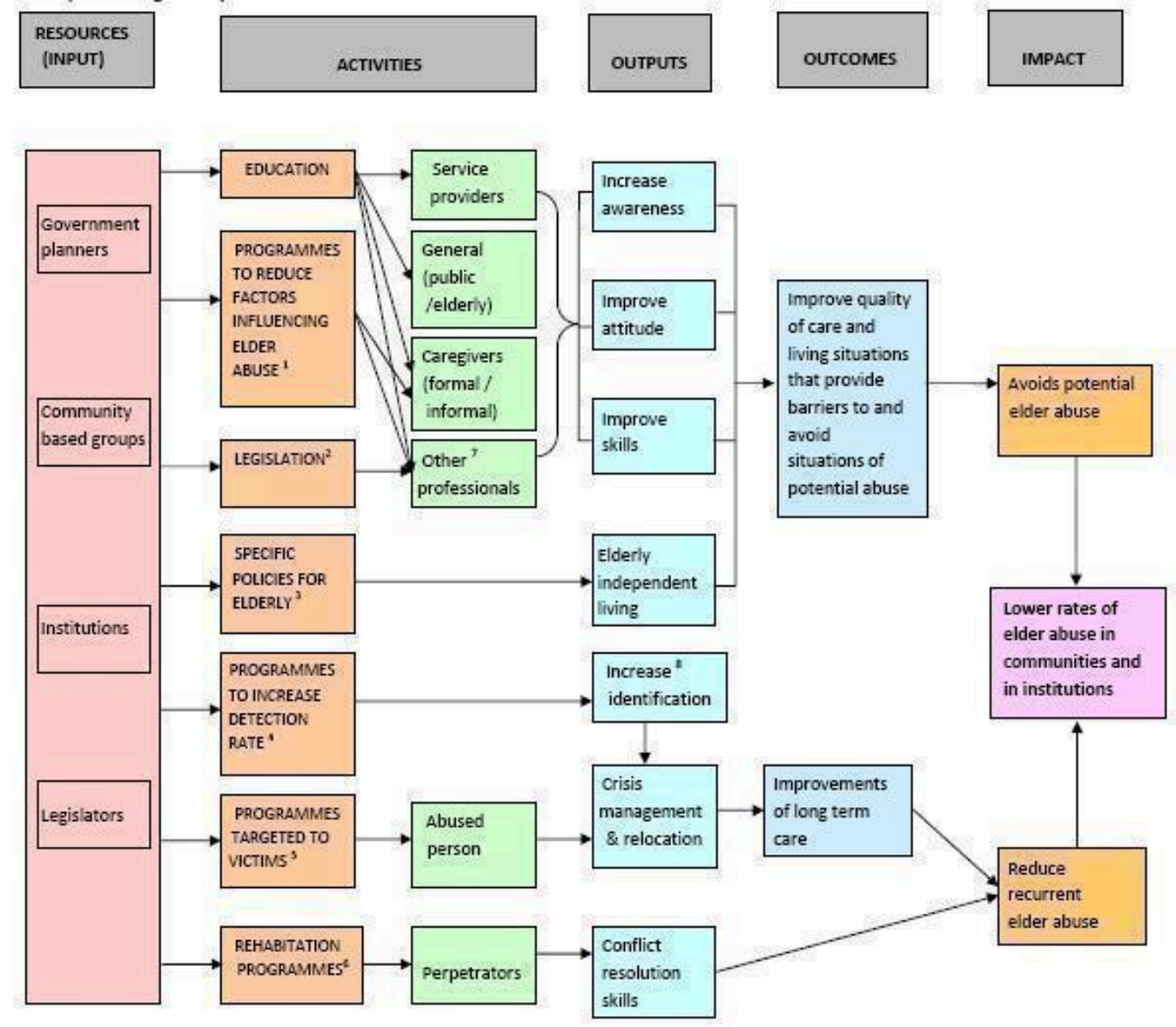

Within the range of interventions, specific 'elderly friendly' policies may be implemented with the intention to strengthen and improve elderly welfare, economic and social standing, which decrease their dependency. These policies may consist of financial independency, welfare assistance, employment opportunities and poverty reduction, involving the financial or banking industries, health sectors, government planners and religious organisations. To achieve this, financial incentives and compensation are provided, which include direct payments to families through cash grants or vouchers to purchase services. Tax incentives for caregiving include deductions and credits. In UK and New Zealand, various banks released the statement of intent on agefriendly banking practices for vulnerable customers (BBA 2010; NZBA 2007). Further, the health sector is encouraged to engage with outside sectors, particularly city councils, urban planners and politicians designing the urban environments in highly innovative age-friendly cities that suit the ageing populations (Heathcote 2011). Some countries have specific employment policies to protect older people. In England, the Independent Safeguarding Authority ensures that employers report a dismissed employee or volunteer for causing serious harm to a vulnerable adult and the employee is then barred from further such employment. Employers need to undertake the Criminal Records Bureau (CRB) checks for healthand social care-related employment (UK Home Office 2012). In addition, initiatives to reduce poverty and social exclusion have been organised, such as the Coming Home Program in United States creating affordable assisted living facilities for lower-income older and frail persons eligible for Medicaid services in rural areas since 1992 (Jenkens 2005; NCBDC 2012). 
Secondary intervention involves close monitoring of vulnerable older adults, early detection of elder abuse through screening or intervening through mandatory reporting, with the assumption that this will avoid recurrence. Monitoring of vulnerable older adults is possible through screening, home-visiting, and homebased geriatric assessment. Helplines (or hotlines) aim to provide victims with the opportunity to report abuse and seek further support, as well as obtain information or referrals to local and national support services. By increasing screening activities, training and education programmes are targeted at health and social care professionals who come into routine contact with older people and are in an ideal position to detect those at risk of, or already experiencing, maltreatment. However, professionals were found to have little insight or guidance for deciding and making judgements regarding abuse of older people, particularly when faced with complex family and contextual factors and ethical dilemmas (Killick 2009). Training programmes are provided with the intention of increasing professional awareness of the various types of elder abuse and their signs and symptoms, and to improve their ability to identify and manage suspected cases effectively (Shefet 2007). They are incorporated in formal curriculum (Wagenaar 2009) or delivered through training courses, workshops (Day 2010), online (Smith 2010) or via printed learning materials (McGarry 2007; Richardson 2002). Although numerous evaluations of training and education programmes have been conducted, they varied substantially in quality, with their effectiveness for the victims showing mixed results. There is currently no such review of the effectiveness of educational interventions in preventing or reducing elder abuse (Day 2010; Richardson 2002).

Legal provisions, including mandatory reporting and adult protection statutes, have been established with the intention of increasing reporting and ending elder abuse (American Bar Association 2006). Disclosure of abusive situations to a legal authority by the affected elderly are impeded due to physical or psychological impairments, poor communication skills, fear of institutionalisation and retaliation, fear of shame or embarrassment, or dependency on the perpetrator (Desmarais 2007). Many states in United States now require mandatory reporting by any persons or specific professionals, such as physicians, psychologists, nurses, law enforcement officials and clergy, to increase reporting (Koenig 2005). Also, laws that govern the licensing and certification of institutions and prohibit healthcare fraud have been introduced to assure the quality of care of nursing home residents and their protection from mistreatment (Gittler 2008). Some laws, especially mandatory reporting, have been implemented with the assumption that they will reduce abuse. However, it has been a contentious issue with many questions raised about its effects; indeed its actual efficacy has yet to be determined (Bonnie 2003; Fulmer 2008a).

When abuse is recognised, it seems logical that referrals need to be made early and adequate follow-up arranged. Tertiary efforts tend to focus on dealing with the immediate consequences of elder abuse, providing support to victims and punishing the offenders rather than preventing abuse in the short and longer term. Thus, temporary placement, adult protective services, emergency shelters, counselling and assistance via support groups targeted at protecting and monitoring victims are widely developed in several countries (Doe 2009; Koenig 2005; Kurrle 2008; Penhale 2008; Podnieks 2008). For example, South Korea has established a centralised system that includes 24-hour emergency hotline for reporting elder abuse, five-day respite programs for caregivers, 15-day temporary residential services for elders and establishment of elder abuse prevention centres (Doe 2009). Multidisciplinary response teams are created in addition to existing adult protective services to respond more efficiently to cases of elder maltreatment. These include forensic centres; vulnerable adult or financial abuse specialist teams; and elder maltreatment prosecution units, or a prevention team that raises awareness of elder maltreatment in the community (Dyer 2005; Schneider 2010; Twomey 2010). For substantiated maltreatment cases, although criminal and civil actions will be undertaken against alleged perpetrators, rehabilitation programmes such as counselling, psychiatric intervention and legal assistance are also available to the perpetrator in some countries (Lithwick 2000).

\section{Why it is important to do this review}

Successful responses to elder abuse involve a public health approach that accounts for the magnitude of the problem, its risk factors and the evidence base of what works that subsequently can be implemented on a wider scale at individual or population levels (Lachs 2004). One major barrier to successful responses to any type of violence, including elderly abuse, is that prevention programmes have been developed in isolation (WHO 2002b). Greater emphasis needs to be placed on undertaking evidence-informed approaches to addressing elder maltreatment. While interventions have been initiated in health, social and legal settings to prevent or reduce elder abuse, little systematic research has been devoted to combining all current evidence available worldwide. There are some initial efforts to gather such evidence (Ploeg 2009), but less so in developing countries. In their review, Ploeg and colleagues found that there were no significant differences in case resolution and rates of recurrence of abuse among the elder abuse interventions evaluated (Ploeg 2009). However, their findings may be limited in the extent to which they can be generalised due to the exclusion of unpublished research reports, non-English language studies, recent studies from developing countries and lack of formal qualitative assessment of included studies. This current review intends to address this gap, reduce the fragmentation in research and improve the evidence base of the actions needed to prevent maltreatment.

\section{O B J E C T IVES}

The primary objective of the review was to assess the effectiveness of primary, secondary and tertiary intervention programmes utilised to reduce or prevent, or both, elderly abuse in organisational, institutional and/or community settings (i.e. their own or someone else's home). We sought to identify and report on adverse consequences or effects of the intervention/s in the review.

The secondary objective was to investigate whether the intervention's effects are modified by types of abuse, types of participants, setting of intervention, or cognitive status of the elderly. 


\section{METHODS}

\section{Criteria for considering studies for this review}

\section{Types of studies}

We included all randomised controlled studies (RCTs) comparing the use of strategies for the prevention and reduction of recurrent elder abuse with a minimum follow-up of 12 weeks in communitydwelling and institutionally cared for elderly persons. Given that elder abuse interventions may involve an entire community or city, quasi-experimental designs such as cluster-randomised controlled trials which use a comparison control population were included. Although the inclusion of non-RCTs increases the susceptibility for bias, we included non-RCTs such as interrupted time (ITS) studies, controlled before-and-after studies (CBAs), and those with comparator groups because a wide variety of approaches and designs have been used in elder abuse interventions and we anticipated that a limited number of RCTs would be available.

We included studies that compared the use of an intervention to prevent elder abuse in one group versus the use of no prevention in the other. Within this, the intervention component of included studies could be a one-off intervention or an intervention extending over a specified length of time. We only included studies that measured elder abuse occurrence (using standardised subjective or objective tools) pre- and post-intervention.

\section{Types of participants}

The target population was elderly people living in the community as well as those being cared for in an institution. We included studies of elderly persons (60 years and older) living in communities (their own or someone else's home) or institutions (such as residential care, health facilities or shelters, prisons or detention centres). This is based on the UN agreed cutoff for the older population (United Nations 2012). Studies that focus on interventions to prevent other crimes against older people by those without a relationship or care responsibility for them (e.g. street mugging or robbery) were excluded.

\section{Types of interventions}

We defined elder abuse prevention intervention (EAPI) as 'any strategies that avoid potential elder abuse or reduce recurrent elder abuse' to lower rates of elder abuse in communities and in institutions. The resources could be provided by government planners, community-based groups, institutions and legislators. As EAPIs could be applied in a number of settings, we used the logic model (Figure 1) to classify the type of intervention and the level (community or individual) in which it is utilised.

The following are examples of EAPIs that we thought might be utilised in order to avoid potential elder abuse or reduce recurrent elder abuse in communities and institutions, consistent with the logic model included in this review. In addition, these included studies improving the quality of care and living situations that provide barriers to situations of potential abuse; and programmes that bring about improvement to long-term care that reduce recurrence of elder abuse. The following interventions were eligible within the defined scope of the review.

\section{Education}

- Training and professional development to service providers on elder abuse.

- Education to the public and elderly, caregivers and other professionals to increase awareness, improve attitudes and build skills for prevention.

\section{Programmes to reduce factors influencing elder abuse}

- Interventions that reduce risk factors, e.g. psychological programmes (anger and stress management), behavioural therapy, provision of respite care and social support groups for caregivers.

- Restraint reduction programmes and institutional policies to limit the unnecessary use of physical restraints.

- Intergenerational programmes to create positive attitudes towards the elderly.

\section{Specific policies on elder abuse}

- Elder abuse-related policies such as those that seek to improve housing, transport, aged-friendly cities, banking, pension management and financial aid that lead to improvements in independent living and welfare.

\section{Legislation on elder abuse}

- Legislation resulting in advocacy-based programmes, laworientated programmes and legal provisions such as mandatory reporting, adult protection statutes, and specific laws to protect whistle-blowers, specifically on elder abuse.

\section{Programmes to increase detection rate for prevention of elder abuse}

- Programmes that attempt to increase the detection rate, such as home visits, home-based geriatric assessment, helplines, training for healthcare and social workers and guidelines and protocols for screening.

\section{Programs targeted to victims of elder abuse}

- Programmes for victims, such as adult protective services, emergency shelters, temporary residential services as crisis management and relocation for improvements in long-term care.

\section{Rehabilitation programmes for perpetrators of elder abuse}

- Programmes of rehabilitation for perpetrators, such as legal assistance, psychiatric intervention, support and counselling that may involve conflict resolution skills.

In this review, education-based interventions were grouped together prior to combining the data. Other modes of interventions were studied as individual programmes due to the differences in the approaches used, motives, content and targeted groups.

\section{Types of outcome measures}

The following are the primary and secondary outcomes pre-defined in this review. 


\section{Primary outcomes}

A primary outcome is any measure of rates of elder abuse in either communities or institutions. They could be further classified as the following, due to the intervention effort (as specified in Figure 1):

- incidence of elder abuse (new instances of abuse occurring);

- recurrence of elder abuse (a second or subsequent episode of abuse occurring).

The definition of incidence of abuse included physical, sexual, emotional, financial abuse, and neglect. Abuse could be assessed using self-report measures (e.g. Conflict Tactics Scale, Elder Abuse Assessment Instrument, Elder Abuse Suspicion Index, Indicators of Abuse screen, Elders Psychological Abuse Scale, or as defined by the authors), medical records, number of protection orders sought, calls to police or police records filed. Incidence may be reported as a frequency count, a rate, or a proportion, but must be for a defined population within a specific period of time (Porta 2014).

\section{Secondary outcomes}

Secondary outcomes included those that may be related to elder abuse behaviour or that explain how interventions work to improve quality of care and living situations, as well as elderly long-term care and those that reduce the potential for elder abuse.

Participant-related outcomes such as:

- increase in awareness regarding elder abuse;

- improvement in attitude towards elder abuse;

- improvement in skills towards handling elder abuse;

- increase in detection;

- increase in elderly independent living.

Victim or perpetrator-related outcomes which include:

- improvement in crisis management and relocation of the victims;

- improvement in conflict resolution and management of the perpetrators.

We reported any adverse outcomes from interventions; where any such events occurred, these were recorded and discussed in the narrative summary.

\section{Search methods for identification of studies}

\section{Electronic searches}

We searched relevant multiple databases and websites (as recommended by Armstrong 2011) using a sensitive search strategy developed by review author PB in liaison with the Public Health Group's Information Specialist and Queensland University of Technology's librarians, and then tailored the MEDLINE strategy for each database during 2015 and then again in early 2016. We handsearched all studies identified in the reference lists of review articles and contacted experts in the field for other potentially eligible studies. We imposed no language in our search. All publications dated from 1975 to present were searched.

The search strategies used to search each database and the dates of search are delineated in Appendix 1.

We searched the following databases.

\section{Health}

- MEDLINE

- Embase

- CINAHL

- Psycinfo

- LILACS

- Proquest central

- Web of Science

- EPPI centre databases - e.g. BiblioMap, DoPHER, TRoPHI

- the Cochrane Library including Central Register of Controlled Trials (CENTRAL) and CRD

- InfoBase

\section{Legal \& Social sciences}

- Sociological abstracts

- Social Science abstracts

- Social Services abstracts

- ASSIA

\section{Grey literature, unpublished research}

- Health Management Information Consortium (HMIC)

- OpenGrey

- Proquest Dissertations and Thesis

- Web of Science

- ZETOC

In addition, we searched the WHO International Clinical Trials Registry Platform (WHO ICTRP) and Clinicaltrials.org to identify studies in progress.

\section{Searching other resources}

In addition to databases, we searched other resources for published and unpublished studies.

We searched the reference lists of all papers and relevant systematic reviews that were identified as meeting the inclusion criteria for the review.

We conducted a Google Scholar search for relevant material and search key websites (International Labour Organisation, WHO and International Network of Agencies for Health Technology Assessment, National Guideline Clearinghouse, Joseph Rowntree Foundation, AgeConcern) and relevant global social/health government departments such as Department of Health in the UK, Australia, etc. The full list of the key organisation websites are presented in Appendix 2. .

We contacted subject experts through the International Network for the Prevention of Elder Abuse, The European Reference framework Online for the Prevention of Elder Abuse and Neglect and the National Center on Elder Abuse in the United States.

\section{Data collection and analysis}

\section{Selection of studies}

We imported article records identified through database searching, websites and other approaches from each database into the bibliographic software package Endnote $X 7$, where duplicates were 
removed and potentially relevant articles selected. We undertook an initial screening of titles and abstracts to remove those which were obviously outside the scope of the review; a task divided between the review authors (WYC, NH, PB) and a research assistant. We were intentionally over-inclusive at this stage and, if in doubt, we included the paper for further consideration. The full text for the papers potentially meeting the inclusion criteria (based on the title and abstract only) were then obtained, and then multiple publications and reports on the same study were linked together. There was no blinding with respect to authors' names, journal or date of publication during this process. Three review authors (WYC, NH, PB) initially independently screened all the fulltext papers which were obtained and, utilising the logic model (Figure 1), assessed whether basic components of the definition of an intervention for preventing abuse and permissible study designs had been fully met. Where there was a persisting difference of opinion, review authors SO and DF reviewed the paper in question in order to reach a consensus between the review authors. We maintained a record of the outcome of the study assessment process for all reviewed material. After the initial selection for full-text review, DF and PB performed a re-screening of a random $10 \%$ of all excluded titles to ensure no suitable titles had been omitted.Subsequently, DF then independently reviewed all potentially included studies, the results were compared and disagreements were identified, discussed and a consensus of included studies reached. We recorded the selection process in detail to complete a study flow diagram (Figure 2). 
Figure 2. Study flow diagram.

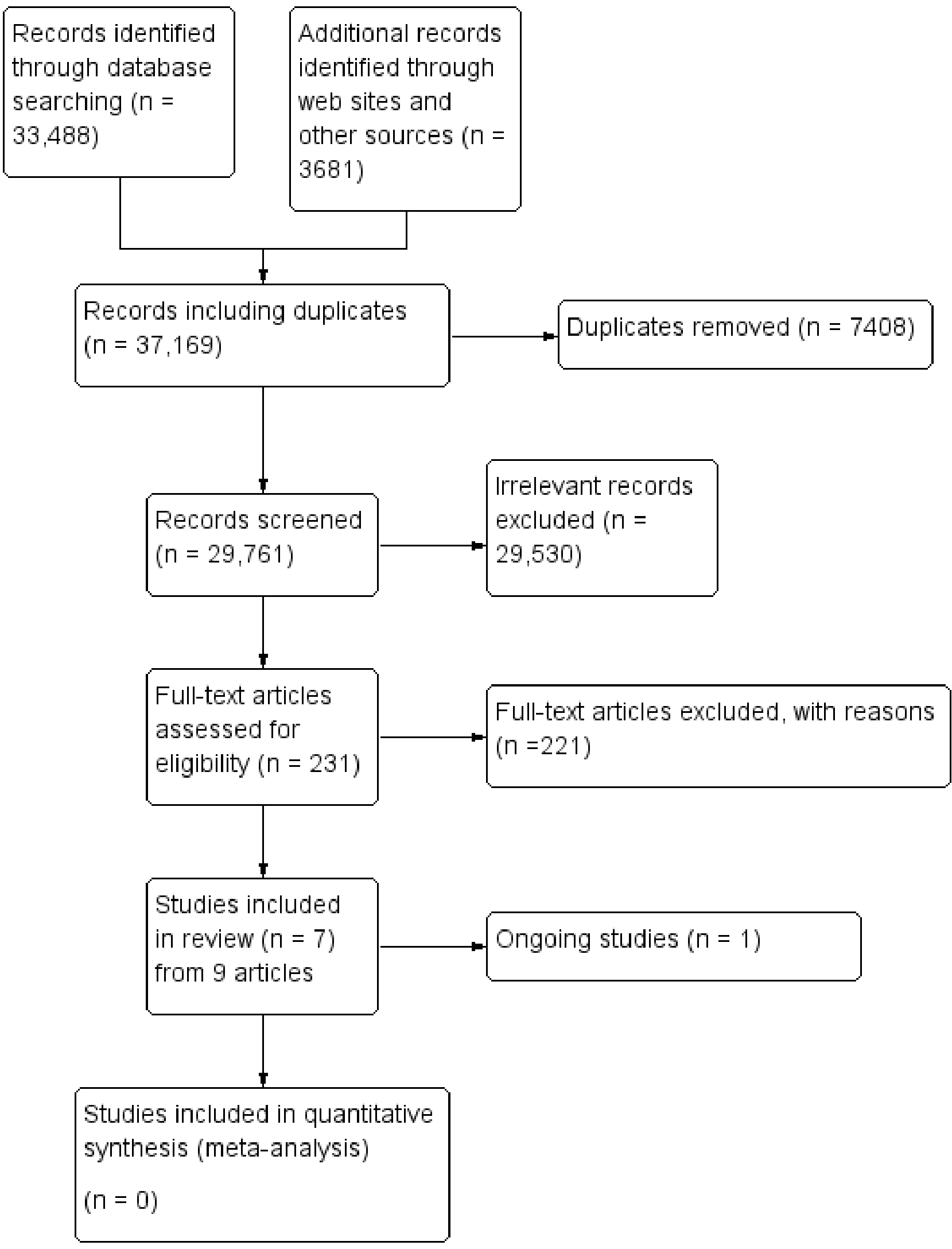




\section{Data extraction and management}

We developed a data extraction form based upon the 'Data Extraction and assessment form' of the Cochrane Public Health Group (CPHG 2011)

Two review authors (WYC and $\mathrm{NH}$ ), independently completed a data extraction form for each study, tailored to the requirements of this review. WYC, DF and PB piloted the data extraction form to assess its ability to capture study data and inform assessment of risk of bias. We resolved any problems identified through discussion and we revised the form, as required. Where studies reported more than one endpoint per outcome, we extracted the primary endpoint identified by the authors. Where the study did not identify a primary endpoint, we ranked the measures by effect size and extracted the median measure (Curran 2007). For any relevant study reported in languages that could not be translated by the review team, WYC completed the data extraction form in conjunction with a translator. We did not find any studies that required further translation.

A checklist was used to ensure inclusion of data relevant for health equity based on PROGRESS-Plus criteria so disadvantaged could be considered in terms of place of residence, race or ethnicity, occupation, gender, religion, socio-economic position, social capital, age, disability and sexual orientation (Ueffing 2011).

In addition, multiple reports and publications of the same study were assembled and then compared for completeness and possible contradictions. We used the logic model (Figure 1) by marking the specific components present in the primary paper and companion publications to assist in the categorisation of studies and interpretation of results, where heterogeneity was present. We managed numerical data that were extracted from the included studies for analysis using a Microsoft Excel spreadsheet.

WYC and NH with the support of PB and DF cross-checked the completed data extraction forms for consistency, and where any discrepancy arose, we achieved consensus through discussion as a complete review team. WYC undertook responsibilities for filing and storing all copies of studies undergoing data extraction and completed data extraction sheets (including printed versions of electronic forms) in a filing cabinet for auditing and checking purposes. Data collated was transferred from our data extraction sheets to RevMan 5.1 (RevMan 2011); NH independently checked the accuracy of this procedure. Where necessary, we contacted study authors to seek provision of data that appeared to be missing from the study reports or to resolve any uncertainty about reported information. We recorded any study that underwent the data extraction process. Studies that did not meet the eligible criteria where examined further and then listed in the Characteristics of excluded studies table with the reason for exclusion noted. All relevant information for the included studies was entered in the Characteristics of included studies table.

Using the location of the intervention, we planned to categorise studies as occurring in low-, middle- and high-income countries, as determined by the World Bank classification. However, we did not find any studies from low- or middle- income countries in this review.

All papers and reports of included studies were reviewed to identify whether any description of costs or resources were made by the authors. Information extracted included descriptors of cost to deliver the intervention over the time specified. Where possible, we intended to separate the cost of the intervention from the cost of the evaluation and research components. Where the results were presented at a population level, we planned to calculate the cost per person. This approach included identifying and including inkind support. We also sought to extract general statements (e.g. "low-cost intervention") made by the authors, where no expression of monetary value was made. Two of the studies included in this review conducted some forms of costing analysis of their intervention, however details of the costing components were not reported in their studies.

\section{Assessment of risk of bias in included studies}

Two review authors (WYC and $\mathrm{NH}$ ) independently assessed the risk of bias in each study using the Cochrane 'Risk of bias' tool. This primarily included the assessment of sequence generation; allocation concealment; blinding of participants and personnel; blinding of outcome assessors; incomplete outcome data; selective outcome reporting; and other sources of bias when evaluating RCTs (Higgins 2008).

In addition, we used the Effective Practice and Organisation of Care (EPOC) 'Risk of bias' tool to assess the risk of bias of nonrandomised studies. For the analysis of non-RCTs, we assessed studies for the five general domains of bias: selection, performance, attrition, detection, and reporting, as well as for an additional category to capture any other concerns pertaining to the study's risk of bias. Eleven questions appropriate for the included study designs were identified from the EPOC tool (EPOC 2015) as shown in the Risk of bias in included studies table. Each question was assessed with answers of 'Yes' indicating low risk of bias, ' $\mathrm{No}$ ' indicating high risk of bias, and 'Unclear' indicating either lack of information or uncertainty.

All eligible studies were judged as at 'low', 'high' or 'unclear' risk of bias, given an overall consideration of the designs and the potential impact of the identified risks noted in the table for each study that contributed results for that outcome according to the EPOC descriptors. We considered overall the study designs and the potential impact of the identified risks. Where a singular minor methodological issue occurred which was deemed unlikely to change interpretation of the findings, we determined that an overall downgrading of the study to high risk was unwarranted (Baker 2015). Disagreement between review authors in the 'Risk of bias' of assessment, where required, were resolved in a discussion with a third author (SO). A standard and consistent approach to Interpretation was developed by the full review team as the method and interpretation was discussed and reviewed by the team prior to application. A second independent assessment for consistency of interpretation was undertaken by the two remaining review authors (PB and DF) who reviewed all decisions made for each study.

The 'Risk of bias' assessment for each included study is documented in the Risk of bias in included studies table. Two figures were generated: a graph that illustrates the proportion of studies for each assessment criterion and a summary figure that shows the methodological rigour of each study.

\section{Measures of treatment effect}

All dichotomous and continuous data were reported separately as found in the primary research. For studies with continuous 
outcomes, mean and standard deviation (SD) were used. For continuous outcomes, mean differences (MD) were used to analyse changes in outcome between the intervention and control groups where possible. Had the studies reported outcomes using disparate scales, we planned to use standardised mean differences (SMDs), if meta-analysed. The effect sizes for dichotomous outcomes were expressed as relative risks (RR) with 95\% confidence intervals, where feasible were calculated. Alternatively the odds ratio (OR) was reported if provided in the study.

To allow for comparison between studies, and given the important differences between intervention (I) and control (C) groups at baseline, we calculated an adjusted estimate of effect. This calculation is based on the differences between the intervention and control group at baseline, similar to Baker 2015. Therefore, for dichotomous outcomes we calculated the following.

- Adjusted risk difference $=\left(I_{\text {post }}-I_{\text {pre }}\right)-\left(C_{\text {post }}-C_{\text {pre }}\right)$.

- Adjusted relative risk $=\left(I_{\text {post }} / C_{\text {post }}\right) /\left(I_{\text {pre }} / C_{\text {pre }}\right)$.

Confidence intervals (95\%) were calculated using the Wald test.

For continuous outcomes we used the data extracted from the included studies to calculate the following.

- Post mean differences (PMD) $=$ Imean $_{\text {post }}-$ Cmean $_{\text {post }}$

- Adjusted mean difference $=\left[\left(\operatorname{Imean}_{\text {post }}-\mathrm{Cmean}_{\text {post }}\right)-\left(\operatorname{Imean}_{\text {pre }}\right.\right.$ - Cmean pre)]

- Adjusted percentage change relative to the control group = $\left[\left(\left(\right.\right.\right.$ Imean $_{\text {post }}-C_{\text {emean }}$ post $)-\left(\right.$ Imean $_{\text {pre }}-$ Cmean $\left.\left._{\text {pre }}\right)\right) /$ Cmean $\left._{\text {post }}\right]$ $x 100$.

The $95 \%$ confidence intervals could not be calculated using this approach.

In this current review it was not appropriate to conduct a metaanalysis.

\section{Unit of analysis issues}

We planned to include cluster-randomised trials in the analyses as well as individually-randomised trials. We would have adjusted their sample sizes using the methods described in the Cochrane Handbook for Systematic Reviews of Interventions (Higgins 2008) and used an estimate of the intra cluster correlation co-efficient (ICC) from the trial or from a study of a similar population. If ICCS from other sources were used, we planned to conduct sensitivity analyses to investigate the effect of variation in the ICC and reported the results. We consider it reasonable to combine the results from both cluster-randomised and individually-randomised trials if there is little heterogeneity between the study designs. A sensitivity analysis would therefore be performed to investigate the effects of the randomisation unit.

In trials with multiple intervention or control groups, we planned to use weighted, pooled means and standard deviations to generate SMDs in order to avoid statistical problems with non-independence of data that would result from including multiple intervention groups as separate trials. Studies comparing different intervention groups or different intensities of the same intervention, with control group, would be excluded from the meta-analysis, but reported in narrative.
All outcome results are described in the narrative.

\section{Dealing with missing data}

Where data were missing, were unclear, or were not fully reported, we attempted to contact the authors of these potentially included studies for clarification and further information. Attempted contact of authors was primarily via email by searching for most recent email address through a Google search. Although stated in our protocol, we chose not to attempt contact via postal address. If we were unable to trace the authors or information was unavailable from the authors within two months of contacting them, we record the information as missing in the data extraction form. Unobtainable methodological data are documented in our 'Risk of bias' tables and unobtainable statistical data were assessed and managed following the guidance provided in Higgins 2008.

\section{Assessment of heterogeneity}

We found content and methodological diversity between all included studies. The logic model was used in categorising the type of intervention strategies used, the participants and outcome measures assessed. Due to heterogeneity in the study designs employed, the populations in which the interventions were conducted, and the interventions themselves, no meta-analysis was conducted in this present review.

\section{Assessment of intensity}

We categorised the intensity of the elder abuse prevention intervention to assess whether intensity could account for differences that existed in the outcomes between studies. Similar to Baker 2015, the intensity of the intervention was categorised based on the six characteristics and attributes that we hypothesised would be important in understanding differences in the effectiveness of the elder abuse prevention interventions. Specifically, these characteristics included: 1) development of community partnerships and coalition; 2) levels of intervention; 3) reach of the strategies; 4) magnitude of the intervention, the extent of continuous provision of the intervention through the intervention period; 5) description of cost; and 6) statement of intensity.

Two review authors (NH and WYC) independently assessed each characteristic as 'more intensive', 'less intensive', or 'unclear' (Baker 2015). We categorised the overall assessment of intensity for each study as 'high', 'medium', 'low', or 'unclear'. Discrepancies were resolved by discussion.

\section{Assessment of reporting biases}

We considered plotting trial effect against standard error (SE) using funnel plots (Sterne 2011). Given that asymmetry could be caused by a relationship between effect size and sample size, or by publication bias (Egger 1998). However, as no meta-analysis was produced, we did not examine any observed effect for clinical heterogeneity or carried out additional sensitivity tests.

\section{Data synthesis}

The assessment of the effect of different types of interventions was guided by the logic model presented in Figure 1. The protocol stated that meta-analysis would only be undertaken if the studies were considered to be clinically homogenous. The diversity of interventions and outcomes however, and the limitations in the 
quantity and quality of studies meant that it was not appropriate a to conduct any meta-analyses in this review.

Narrative synthesis was therefore conducted with studies categorised using the interventions presented in the logic model. An additional synthesis by the primary and secondary study outcomes already identified in this paper was also conducted. In synthesising the data, the results of any intervention versus no intervention (i.e. control group), immediately post-intervention, and at any intervals within the 12 months follow-up, were used, and where possible reported on the original scale. All data were recorded using an Excel spreadsheet.

\section{Subgroup analysis and investigation of heterogeneity}

There were insufficient studies identified to allow all subgroup analyses to be performed as planned in the protocol for this review. Where sufficient data were available, we planned to carry out the following subgroup analyses based on the following; 1) type of abuse; 2) type of intervention (e.g. primary, secondary or tertiary prevention); 3) cognitive status of elderly (cognitively intact versus impaired); 4) type of setting (e.g. community dwelling versus institutions such as residential care, health facilities or shelters); 5) geographical regions (those from low- middle- or high-income countries); 6) socio-demographic characteristics of the target population (e.g. victims, perpetrators, socio-economic status, gender or others); 7) effect of low follow-up in the studies. Given the absence of trustworthy data or appropriate subgroups reported in these studies, no further subgroup analysis could be undertaken.

\section{Sensitivity analysis}

We had intended to carry out a sensitivity analysis for studies with low risk of bias which were combined, however as no meta-analysis was conducted in this review, this was not performed.

\section{'Summary of findings' tables}

We intended to prepare 'Summary of findings' tables for the primary outcomes related to elder abuse using GRADE profiler (Schünemann 2011), however due to the limited studies which could not be combined, we prepared modified tables. We summarised the quality of evidence by applying the principles of the GRADE framework and following the recommendations and worksheets of EPOC for creating 'Summary of findings' tables (EPOC 2011).

We used four levels of quality (high, moderate, low and very low) to describe the body of evidence. We assessed the quality of evidence for each outcome across studies. We assessed the study design, risk of bias, imprecision, inconsistency, indirectness and magnitude of effect based on GRADE criteria. The primary determinant for upgrading or downgrading the evidence was whether the issues identified were likely to affect the outcome. The ratings of the quality of evidence were modified downward based on study limitations, imprecision, inconsistency of results, indirectness of evidence and likelihood of publication bias. The ratings were modified upward when the study had a large magnitude of effect, existing dose-response gradient, or when consideration of all plausible residual confounders and biases would reduce a demonstrated effect, or suggest a spurious effect when results showed no effect.
We had intended one 'Summary of findings' table to contain a summary statement of the effect of the intervention upon population levels of primary outcomes using three scenarios of elder abuse levels and intervention approaches that are indicative of low-, middle- and high-income countries, however there were insufficient data from low- and middle-income countries to determine this. We also intended to explore if an equity gradient was apparent, such as the staircase effect (Tugwell 2006) and to examine the data to identify whether there could be an increasing gap and decreasing effectiveness by advantaged and disadvantaged populations across relevant components of the intervention. However, the current body of evidence was insufficient to undertake these analyses.

As a meta-analysis was not appropriate for this current review, alternative 'Summary of findings' tables using narrative analysis of the included studies were prepared.

\section{RE S U L T S}

\section{Description of studies}

See Characteristics of included studies; Characteristics of excluded studies; Ongoing studies

\section{Results of the search}

As shown in Figure 2, the electronic searches between 30 August 2015 and 16 March 2016 of the databases yielded 33,488 hits. Web searches and from other sources yielded 3681 additional records. Following the removal of duplicates, 29,761 records remained. After initial screening based on the title, 230 citations were considered potentially eligible and were assessed in full text. Following the review of full-text, seven studies (nine reports) were identified as meeting the inclusion criteria (Bartels 2005; Brownell 2006; Davis 2001; Hsieh 2009; Cooper 2015; Richardson 2002; Teresi 2013) and one ongoing study (Loh 2015), The results of the searches are shown in Appendix 1 and Appendix 2.

\section{Included studies}

Characteristics of included studies. All seven included studies were set in high-income countries according to the World Bank economic classification (USA four, Taiwan one, and UK two).The included studies were grouped into four of the seven categories defined prior to the commencement of the review and as presented in the logic model. Of the seven included studies, three investigated the effectiveness of educational interventions targeting healthcare professionals (Richardson 2002; Teresi 2013), and caregivers (Hsieh 2009). One randomised study evaluated the effectiveness of a programme aiming to reduce factors influencing elder abuse by promoting the mental health of family carers (Cooper 2015). One study evaluated an intervention designed to increase the detection of abuse (Bartels 2005). Two studies targeted victims of abuse; one by providing a psycho-social support and a structured educational programme in a group setting (Brownell 2006), and the other, a 'blended' multi-strategy consisting of a broad community public education strategy followed by active individual social support services and monitoring by police of households (Davis 2001) There were no eligible studies which investigated the effectiveness of rehabilitation programmes for perpetrators of elder abuse, legislation, or specific policies on elder abuse. Summary details of all included studies are found in Table 1. 
Three different study designs were employed in the seven included studies. Five studies were described as randomised with a control or comparison. Four were randomised controlled trials (Brownell 2006; Davis 2001; Cooper 2015 and Richardson 2002) and one was a cluster-randomised trial (Teresi 2013). Randomised trials were used in studies examining the effectiveness of educational interventions $(n=2)$, programmes to reduce risk factors $(n=1)$ and interventions targeted at victims $(n=2)$. Two of the included studies, one investigating educational interventions (Hsieh 2009), and the other programmes to increase detection (Bartels 2005), used controlled before-and-after study designs without randomisation comparing outcomes before and after the implementation of the intervention.

A subjective assessment of intensity was conducted based on the consideration of six criteria as described in the methods section. Two studies were judged to be of high intensity, two of medium intensity and three of low intensity (Table 2). Categorisation of high intensity was typically assigned to an intervention which acted on more than one level within the target population and multiple components as understood by the logic model (Figure 1). For example, Davis 2001 utilised two stages; the first stage targeted the broader public through education, and the second involved participants (victims) who received a visit, and was thus deemed 'high intensity'. Bartels 2005, also a high-intensity intervention, demonstrated a prolonged duration of delivery ( 24 months) with a comprehensive program that incorporated 22 assessments and treatment-planning domains, and involved an extensive number of agencies, and broad participation of clinicians. Cooper 2015, at eight to 14 weeks duration, was of shorter duration than Bartels 2005, however provided a comprehensive approach during the period of intervention. Both Bartels 2005 and Cooper 2015 were deemed 'medium intensity', having intensity scores of six and five, respectively.

\section{Excluded studies}

Of the 230 papers examined in full text, we found 221 that did not meet our inclusion criteria and that we summarily excluded.The Characteristics of excluded studies table lists the studies that were excluded and the determined reasons. In several cases the studies were excluded for more than one reason. The predominant reasons for studies being excluded at this stage of the selection process were ineligible study design (151), no outcomes measures or no outcomes related to elder abuse (45), not about elder abuse (12), no evaluation described (9), no intervention (2), and not an eligible population (2). Three studies were excluded as while they appeared to use an interrupted time series (ITS) design, they did not have at least three data points before and after the intervention that is necessary to be defined as an ITS study.

\section{Risk of bias in included studies}

All included studies were assessed for their risk of bias. We assessed the risk of bias using the Cochrane 'Risk of bias' tool to which we added minor amendments according to EPOC recommendations as outlined in the methods section.The individual assessment and the reasons for the assessment are detailed in the Risk of bias in included studies tables. Graphical presentation of the results of the individual studies and of the overall body of evidence are found in Figure 3 and Figure 4. Assessment using the 'Risk of bias' tool suggests the trustworthiness of the studies across the included body of research was poor. Only one study (Cooper 2015) was judged as having no domains at high risk of bias, with two studies having two (Richardson 2002; Teresi 2013). Across nine of the 11 domains for which studies were judged, at least $40 \%$ of studies were judged as being at high risk of bias. 
Figure 3. 'Risk of bias' summary: review authors' judgements about each risk of bias item for each included study.

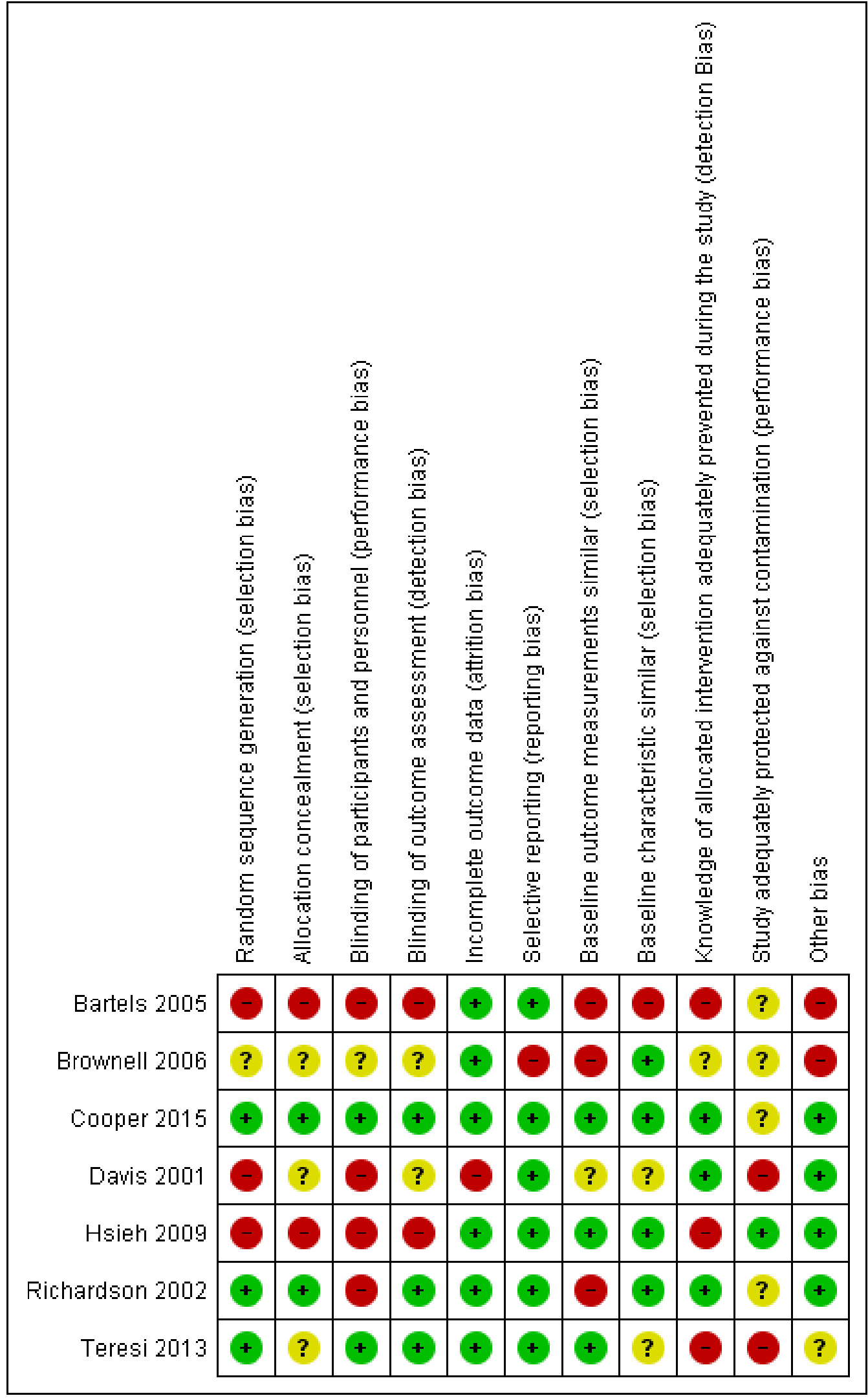


Figure 4. 'Risk of bias' graph: review authors' judgements about each risk of bias item presented as percentages across all included studies.

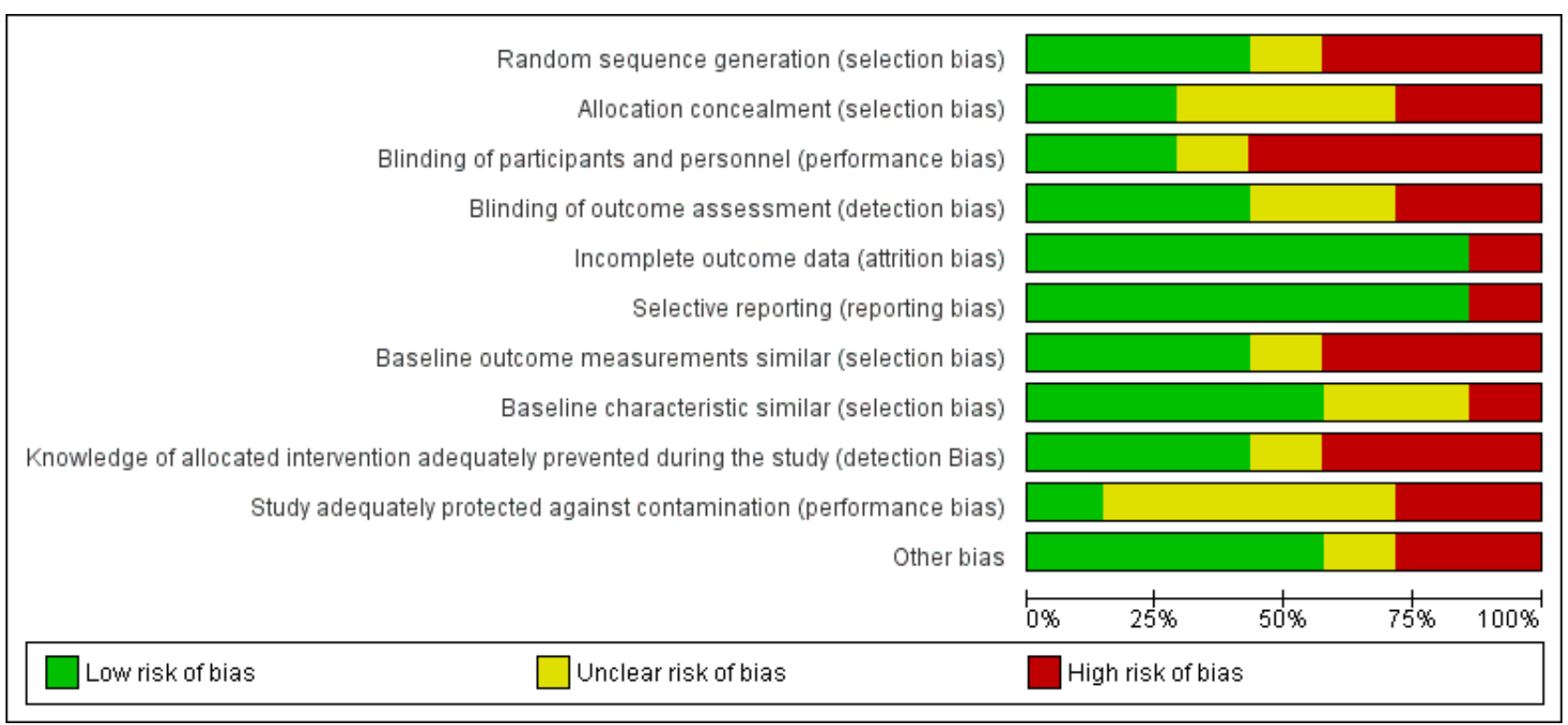

General agreement between authors assessing the risks of bias in the studies was high with consensus reached quickly through discussion when queries arose.

\section{Allocation}

Selection bias was expected to be relatively low as five of the seven studies were described by the authors as 'randomised'. However, only three ( Cooper 2015; Richardson 2002; Teresi 2013) of the five randomised studies described an adequate method of sequence generation. Allocation concealment was even more problematic, in that only two of the seven studies $(29 \%)$ were judged as low risk for selection bias (Cooper 2015; Richardson 2002). This was also made more problematic for one study for which the baseline characteristics were not comparable for the intervention and control (Bartels 2005). If those selected for treatment were reversed, there is no assurance the outcome would be the same and thus the findings and conclusions could not be deemed trustworthy. For example, in Bartels 2005 the intervention group had large, medium and small amounts of caseloads, whereas the control had only small and large caseloads. Further, there were also differences in the average number of clinicians and average elderly persons between the intervention and comparison groups.

\section{Blinding}

Blinding effects both measurement bias and performance bias. In these studies, blinding of the participants was generally inadequate to minimise these biases. Two (Cooper 2015; Teresi 2013) of the seven studies blinded both participant and the personnel performing the intervention. We judged four studies at high risk of bias (Bartels 2005; Davis 2001; Hsieh 2009; Richardson 2002) given the lack of blinding for both those providing the intervention and for the participants (a combined assessment). Although Richardson 2002 did not blind interventionists, the participants' outcome assessment was blinded as the tutor and persons undertaking the rating were blinded as to who was participating in the study. On this aspect, there were two studies where the risk of bias associated with outcome assessment (detection bias) was at high risk (Hsieh
2009 and Bartels 2005), and two were the outcome assessment was unclear (Brownell 2006; Davis 2001). The knowledge of the allocated intervention within the context of outcome assessment resulted in a high risk of bias in three studies (Bartels 2005; Hsieh 2009; Teresi 2013). In the case of Teresi 2013, this was related to an inability to blind the certified nursing group.

\section{Incomplete outcome data}

The data were generally complete for the included studies and this was one of the two domains with better scores. Only one study (Davis 2001) was judged at high risk of bias. Davis 2001 experienced dropout rates in the control group ranging from $35.1 \%$ to $39.7 \%$, and in the home-visiting intervention group ranging from $25.8 \%$ to $29.7 \%$. The remaining six studies were all judged at low risk of bias for this domain.

\section{Selective reporting}

Selective reporting of outcome bias was relatively low. Only one study was judged at high risk of selective reporting (Brownell 2006), with six studies being seen as being at low risk of bias for this domain. The outcomes generally aligned with the intent of the study, although an absence of trial registration and publication of protocols made this more difficult to assess. In the one study assessed as high risk of bias, Brownell 2006, one of the secondary measures (self-esteem) was not reported.

\section{Other potential sources of bias}

As discussed in the Methods section, the inclusion of nonrandomised studies in this review prompted the assessment of additional 'Risk of bias' domains. Of concern, overall, $40 \%$ the studies were judged as being at high risk of bias when considering similarity between groups at baseline, prevention of knowledge of allocation concealment during the study, protection against contamination, and other biases. Most studies failed to describe efforts aimed at preventing contamination where it may have been possible to occur (e.g.Bartels 2005; Brownell 2006) resulting in an 
assessment of "unclear" risk of bias for more than half of the studies. High risk of bias from continuation was apparent in Davis 2001 where a cross-over of participants occurred during the study. More complicated, Teresi 2013 involved randomisation of units within the same facility, and thus there was potential for the control groups to also receive the intervention.

\section{Effects of interventions}

See: Summary of findings for the main comparison; Summary of findings 2; Summary of findings 3; Summary of findings 4

As reported previously, the nature of the studies included in this review, as well as the outcomes and data reported meant that it would be inappropriate to conduct any formal statistical pooling of studies of the primary outcome measures of occurrence or recurrence of abuse. The synthesis presented here is therefore predominantly narrative with findings organised by elder abuse preventive initiative (EAPI) as defined in the methods and presented in the logic model (Figure 1). Within these categories, findings from randomised and non-randomised studies have been presented separated. To help the reader appreciate the evidence around individual outcomes, we also synthesised findings around the primary and secondary outcomes.

For each of the EAPI's for which there were included studies, a 'Summary of findings' table was developed (Summary of findings for the main comparison; Summary of findings 2; Summary of findings 3; Summary of findings 4). In addition, details of the design, sample size, population included, country of study, and 'Risk of bias' domains judged to be at low risk of bias of the individual studies have been summarised in a separate table (Table 1 ).

\section{Educational Interventions for health practitioners and/or carers}

Three studies investigating the effects of educational interventions were included in this review. Of these, two were randomised studies (Richardson 2002; Teresi 2013), and one was a controlled before and after study (Hsieh 2009). Two of the interventions included in this category were aimed at health practitioners (nurses, trainee psychiatrists, care assistants etc), while one was aimed at caregivers (Hsieh 2009). Findings are summarised in the Summary of findings for the main comparison, however we were unable to perform any meta-analysis given the differences in interventions, populations and outcomes used in the included studies, and therefore the results of the studies are presented below individually. Each of the studies did however measure knowledge and found that through their interventions they were able to improve knowledge relevant to elder abuse.

\section{$\underline{\text { Randomised studies }}$}

In Richardson 2002, a study having two items judged as being at high risk of bias, the investigators aimed to determine the effectiveness of attending an educational course compared to printed educational material in improving the management of abuse of older people by nurses, care assistants and social workers. Participants were randomised to receive either an educational course $(n=44)$ or reading material (comparison) ( $n=42$ ). Outcomes were measured using a knowledge and management questionnaire based on vignettes of realistic or actual scenarios, given pre- and post-intervention (KAMA -knowledge and management). The authors reported a significant difference between groups at baseline with those receiving the educational course having significantly higher mean KAMA scores $(P=0.0001)$. Post-intervention results indicated that those participating in the educational course improved (3.7), while those who received the material declined (-2.9), with an adjusted mean difference of 6.6 (95\% confidence interval $(\mathrm{Cl}) 1.97$ to 11.23 ) in favour of the intervention. It was difficult to determine whether this difference was due to the intervention or to the difference in baseline scores. Analysis using ANOVA indicated that the only other significant variables - other then being randomised to the educational course - were low baseline scores. Further, there was no reporting of a difference between the two groups at post-intervention with mean KAMA scores at this time point. The review authors calculated an adjusted mean difference of 6.6 and an adjusted \% change relative to control group of $25.8 \%$.

The intervention group had a higher positive attitude at both baseline and at post-intervention. The adjusted mean difference was 0.2 , and the adjusted $\%$ relative to control group was $3.2 \%$.

For burn-out, there was no significant difference between intervention and control at follow-up (MD1.50, 95\% Cl -6.75 to 3.75). Adjusted mean difference was 0.1 , and the adjusted $\%$ change relative to control group $0.6 \%$.

Teresi 2013 was a prospective cluster-randomised study which sought to evaluate the impact of a training program plus an implementation protocol to increase the knowledge, recognition and reporting of resident-to-resident elder mistreatment (R-REM) in nursing homes. Nursing home units of five large facilities were randomised with nursing staff from 23 nursing homes receiving three modules of training around recognising, and managing R-REM, as well as implementing a best-practice protocol and improving reporting of R-REM. Importantly, the study was deemed to be of unclear risk of selection bias due to inadequate information of allocation concealment. Staff $(n=325)$ in the 24 control units only received training on the reporting form used to collect outcome data regarding the 1405 residents, 685 control and 720 intervention).

At six months the adjusted mean difference for the staff-reported number of incidents in the previous two weeks was 0.82 , and the adjusted \% change relative to control group was 304\%. However at 12 months, the adjusted mean difference was 0.42 , and the adjusted \% change relative to control group $420 \%$.

Staff knowledge (related to R-REM) and frequency of recognition and reported R-REM was measured only for the intervention group thus forming a process description (not an outcome assessment). Follow-up measurements at six and 12 months suggested a significant increase in knowledge of elder-to-elder abuse $(P<$ 0.001 ), significantly increased recognition of R-REM occurring $(P<0.001)$, and significantly increased longitudinal reporting (documentation) as compared with the control group $(P=0.0058)$. Detection bias was high as the assessors had knowledge of the intervention. This was maintained with the experimental group reporting seven times as many incidents at six months and 12 times as many incidents at 12 months with 23 cases detected in the control and 239 in the intervention (Poisson model $P=0.0058$ ). The process evaluation also found that management skills increased for the intervention group.

Non-randomised study 
Hsieh 2009 was a controlled before-and-after study in which 50 caregivers from two nursing homes in southern Taiwan attended eight group sessions of 1 to 1.5 hours length over an eight-week period. Caregivers from two other nursing homes served as the control group $(n=50)$ (112 randomised). The outcomes measured included the Caregiver Psychological Elder Abuse Behavior (CPEAB) Scale for which a high score indicated a higher tendency towards abusive behaviour. The adjusted mean difference was -3.46 and adjusted \% change relative to the control group was $11.4 \%$. Statistically significant differences between the post-test scores of the two groups relative to CPEAB were found $(F=4.02, P=$ 0.048 and 0.018 , respectively). For the Knowledge of Gerontology Scale (KGNS), the adjusted mean difference was 1.32, and the adjusted \% change relative to control group 5\%. For the Work Stressors Inventory (WSI), the adjusted mean difference was 3.2, and adjusted $\%$ change relative to control group of $6 \%$ (overall comparison, $P=0.666$ ). The results suggested a significant difference in the alleviation of caregiver psychological abusive behaviour and improvement in knowledge of elder care, however the trustworthiness of this finding is low as both selection and detection bias were high. There was no difference reported in carer stress.

In summary, across the three studies, it is uncertain that these programs improve knowledge (based on a ' very low' GRADE rating), and if it did, whether it would translate into a reduction of elder abuse. Such programs may be able to improve the ability to detect resident-to-resident abuse (based on a 'low' GRADE rating).

\section{Programme to reduce factors influencing elder abuse}

Only one study investigated a programme to reduce factors influencing elder abuse (Cooper 2015). This low risk of bias study targeted carers of family members suffering from dementia and reported an outcome related to potentially abusive carer behaviour towards those in their care. Summary of findings 2

\section{Randomised study}

The START trial (Cooper 2015), a low risk of bias study, randomised primary carers of family members suffering from dementia (but not living in 24-hour care) to receive eight sessions of a manualbased coping strategy delivered over an eight- to 14-week period ( $\mathrm{n}$ $=173)$ or usual care $(n=87)$. The purpose of this well-conducted study was to evaluate the effectiveness of an intervention designed to promote the mental health of carers of family members with dementia. A modified conflict scale of potentially abusive carer behaviours towards the recipient of their care was utilised for the primary outcome. There was no significant difference between reporting of less abusive behaviour in carers in the intervention group compared to those in the control group at eight months (adjusted OR $0.48,95 \% \mathrm{Cl} 0.18$ to 1.27 ) and at 24 months (adjusted OR $0.59,95 \% \mathrm{Cl} 0.27$ to 1.28 ). Secondary outcomes of anxiety and depression and quality of life of both the carer and the recipient of care were measured at three time points over an eight-week period. The measures of anxiety, depression and quality of life favoured the intervention. For anxiety, the mean total scores on the hospital anxiety and depression scale (HADS) were statistically lower in the intervention group than in the usual care group over the eight-month evaluation period with an adjusted difference in means of -1.80 points ( $95 \%$ confidence interval -3.29 to -0.31 ; $P$ $=0.02$ ) and absolute difference in means of -2.0 points. Health status (carers) was statistically higher (adjusted treatment effect scores $4.55(0.92$ to 8.17$)(n=219))$. Carers in the intervention group were less likely to have case-level depression (OR 0.24, 95\% Cl 0.07 to 0.76 ), and there was not a statistically significant reduction in case-level anxiety (OR $0.30,95 \% \mathrm{Cl} 0.08$ to 1.05 ). Treatment effect reported was adjusted for baseline score and centre: -0.88 ( -1.68 to $-0.09)(n=229)$. Carers' quality of life was higher in the intervention group (difference in means $4.09,95 \% \mathrm{Cl} 0.34$ to 7.83 ), but not for the recipient of care (difference in means $0.59,-0.72$ to 1.89 ). We graded the outcome of occurrence of abuse as 'low quality' as the findings are based on one study which had serious imprecision as it was underpowered for the outcome measures. However, for the combined surrogate outcome of 'anxiety and depression', we graded the evidence as 'moderate quality', indicating that this intervention approach probably reduces anxiety and depression of caregivers. It is unclear whether this translates into less abuse as occurrence is not reported.

\section{Programme to increase detection rate for prevention of elder abuse}

One non-randomised controlled trial at high risk of bias reported on an intervention that included a component to improve the documentation of abuse and neglect which the study reported on as an outcome. Summary of findings 3 .

\section{Non-randomised study}

Bartels 2005 reported on a controlled before-and-after study evaluating the effectiveness of an assessment and service planning intervention for improving the clinical practices of non-physician community mental health providers caring for older persons. The intervention was an integrated system of clinical assessment, decision support, and outcomes measurement process designed to improve assessment practices and service planning for older adults with mental illness. Thirteen community mental health organisations and home healthcare organisations were assigned to intervention or comparison groups. Clinicians in the intervention group received a review of the assessment and service planning methodology. The clinicians from both groups were asked to enrol eligible older adult persons. Only secondary outcomes are reported.

There were no differences in clinician-reported baseline assessment practices for neglect and abuse between the intervention and comparison groups. The analyses compared pre-post change scores between the intervention group and the comparison group and reported an odds ratio of 6.50 (however neither the $P$ value or confidence interval was provided to substantiate the claim that it is 'significant' ( $n=44$ clinicians)). Reanalysis by the review authors found an adjusted risk difference (RD) of 37.2 (95\% Cl -3.5 to 77.9$)$ and adjusted RR of 3.24 (95\% Cl 0.75 to 13.9). Chart reviews at 12 months suggested the intervention was associated with an increase in assessment and documentation of domains relating to abuse including safety, and neglect and abuse.

The study authors stated that there was a significant increase at follow-up in the proportion of charts which documented neglect and abuse in the intervention group (baseline 19.7\%; follow-up $91.8 \%$ ) compared to the comparison group (baseline $0 \%$, follow-up $2.6 \%$ ) ('odds ratio could not be calculated'). Re-analysis of the chart audits by the review authors found an adjusted RD of $69.5(95 \% \mathrm{Cl}$ 62.9 to 71.1) showing evidence of increased documentation. The adjusted RR could not be calculated from the data provided. 
The GRADE of the evidence is 'very low quality' as it represents one study which is at seriously high risk of bias over eight of the 11 items assessed, and lacked transparency in the analysis to support claims made by the authors.

\section{Programmes targeted to victims of elder abuse}

Two randomised studies investigated the effectiveness of programs targeted at the victims of elder abuse. The nature of the interventions, however, were very different (one primarily educational and one involving a psycho-social support group) it was inappropriate to conduct a meta-analysis. The findings have however been summarised in Summary of findings 4 .

\section{$\underline{\text { Randomised studies }}$}

Davis 2001 reported on a nested randomised control trial within the communities of 403 residents who had previously reported an incident of elder abuse. In the first instance, the communities were randomised to receive or not receive a whole community public education program comprising of presentations by police, posters displayed, and leaflets delivered to all elderly persons. At the individual level, abused participants were then randomised to receive or not receive a multi-component intervention consisting of police and social worker visits with support, following up domestic violence complaints and household monitoring by police. Reccurence of elder abuse was measured over an 18-month period. While the home visit intervention produced no difference in victims knowledge of elder abuse issues, their use of social services or their psychological well-being, the outcome for the intervention group was worse than the controls as they were more likely to report new instances of abuse to police and research personnel. The Hazard ratio (HR) from a cox multiple regression analysis was reported: public education ( $H R=1.26$, home visit $H R=2.05$ (alpha level 0.05) and both public education and home visit $\mathrm{HR}=1.78$ (alpha level = $0.01),(n=403)$. As no baseline data are provided for knowledge and the use of services at baseline, no meaningful comparison of the effects of the intervention are available for reporting.

Brownell 2006 was a very small randomised controlled trial in which victims (all mistreated at baseline) were randomised to participate in an elder mistreatment psycho-social support group which included a structured curriculum of learning on domestic violence and abuse and neglect amongst other topics delivered in two-hour sessions for eight consecutive weeks $(n=9)$, or no intervention $(n=6)$. As with the previous study, this small study reported the primary outcomes of recurrence of victim abuse. For physical abuse, post-test: $0 \%$ of controls and $13 \%$ of intervention participants reported abuse $(P=0.41)$. The adjusted RR could not be calculated although the adjusted RD was $34(95 \% \mathrm{Cl}-23.5$ to 91.5$)$. For non-physical abuse, $75 \%$ of controls and $83 \%$ of intervention participants reported non-physical abuse during the study with a comparison between intervention and control groups finding no significant difference $(P=0.71)$, with an adjusted RR $0.91(95 \% \mathrm{Cl}$ 0.58 to 1.45$)$ and an adjusted RD -9 ( $95 \% \mathrm{Cl}-56.8$ to 38.8$)$.

There was no statistically significant difference between the groups for depression as indicated by the adjusted RR (adjusted RR .42, (95\% Cl 0.05 to 3.7), an adjusted RD (adjusted RD -19 (95\% Cl -60 to 22.0$)$ ). Guilt was also not statistically different ((adjusted RR 1.33 (95\% $\mathrm{Cl} 0.30$ to 5.89 ); and adjusted RD 3 ( $95 \% \mathrm{Cl}-11.5$ to 17.5$)$ ).
There was Insuffcient reporting of Self-Esteem (Rosenberg scale), and the findings for Sense of Control and Social Support, anxiety and somatisation were not reported.

No firm conclusions can be drawn from this study due to its small size and high risk of bias on eight of the 11 categories assessed.

\section{Rehabilitation programmes for perpetrators of elder abuse}

There we no eligible studies of programmes for perpetrators.

\section{Primary outcomes}

The details of the primary outcomes measured by the included studies are provided in Table 3 and Table 4, and described earlier in Effects of interventions. Two of the included studies used measures or proxy measures for outcomes relating to the occurrence of elder abuse (Cooper 2015; Hsieh 2009), and two studies measured the recurrence of abuse (Brownell 2006; Davis 2001). The tools and methods used to measure these outcomes varied between studies as did the interventions which the studies evaluated.

Of the studies measuring the occurrence of elder abuse, only Hsieh 2009, a non-randomised study at high risk of bias, examining an educational intervention in caregivers in nursing homes in Taiwan, reported a between-group effect of a net decrease in abusive behaviours, as measured through the Caregiver Psychological Elder Abuse Behavior Scale (between-group $F=4.02, P=0048$ ). Using the data provided, the mean difference in the post-test measures for intervention and control was $-1.22(95 \% \mathrm{Cl}-13.5$ to 1.10$)$. In a randomised trial, at low risk of bias, the post-mean difference of an intervention intended to promote the mental health of family carers showed no significant difference in the reporting of less abusive behaviour in carers in the intervention group (Cooper 2015).

Two studies investigated interventions which targeted victims of abuse. A trial of an intervention which included a home visit by a police officer and a social worker to victims of elder abuse reported an increase in the occurrence or reporting of abuse in the intervention group over a 12-month follow-up (Davis 2001). A small randomised study $(n=14)$ evaluating the effectiveness of a psychosocial support group with relevant curriculum, found no difference on re-occurence of abuse between the two groups (Brownell 2006).

\section{Secondary outcomes - participant-related outcomes}

Secondary outcomes reported in the included studies are detailed in Table 5, and described earlier in the Effects of interventions section.

\section{Increased awareness regarding elder abuse}

No studies included outcomes relating to increasing the awareness of elder abuses.

\section{Improvement in knowledge and attitude towards elder abuse}

Several studies included outcomes that related to the knowledge and attitude of carers towards elder abuse. Educational interventions, appeared to be broadly effective at increasing knowledge regarding elder abuse with Hsieh 2009, Richardson 2002, and Teresi 2013 all reporting an increase in knowledge in health professionals included in their studies. Richardson 2002 also reported on attitudes of staff towards demented patients, and while 
there was no difference brought about by the intervention, it was noted that pre-intervention scores were high, so no improvement would have been expected.

\section{Improvement in skills towards handling elder abuse}

While some studies included measurement of reporting or detection behaviours, skills were not measured in any of the trials included, with the exception of Teresi 2013, which demonstrated a gain in knowledge of management of resident-to-resident elder mistreatment after an educational module in the intervention group, but this was not compared to the control.

\section{Increased detection}

The concept of measuring improvement in detection or reporting as opposed to the occurrence or recurrence of abuse is complicated. Nonetheless, Bartels 2005 and Teresi 2013 included outcomes related to the detection or reporting of elder abuse. The educational intervention evaluated by Teresi 2013 was effective at improving recognition and longitudinal reporting of resident-toresident abuse.

In a study designed to improve detection through the improvement of mental health screening and service planning practices by clinicians for older adults, Bartels 2005 found that an assessment and service planning intervention was associated with an increase in assessment and documentation of domains relating to abuse including safety, and neglect and abuse.

\section{Increase in elderly independent living}

No studies measured elderly independent living

\section{Secondary outcomes - victim- or perpetrator-related outcomes}

\section{Improvement in crisis management and relocation of victims}

No studies measured improvement in crisis management or relocation of victims.

\section{Improvement in conflict resolution and management of perpetrators}

No studies measured improvement in conflict resolution and management of perpetrators.

\section{More intense studies}

Four of the studies included in the review were classed as being medium to highly intensive based upon the subjective assessment in the methods section (Bartels 2005; Cooper 2015; Davis 2001; Teresi 2013). Of these studies, Bartels 2005 did however show some effect, but because of high risk of bias of the included studies, incompleteness of reporting, inconsistency of the effectiveness, and the heterogeneity observed in the intervention approaches, no firm conclusion can be drawn.

\section{DISCUSSION}

\section{Summary of main results}

Seven studies met the criteria for inclusion in this review; five of which were described as 'randomised trials'. These seven studies investigated the efficacy of interventions aimed at decreasing the occurrence or recurrence of elder abuse by acting on mechanisms believed to be capable of moderating long-term outcomes. Five of the studies sought to modify the behaviour of carers, family members, other service providers, or victims of abuse through the provision of a variety of programmes. There is some evidence to suggest that the interventions were able to improve knowledge and attitudes relevant to elder abuse; however their ability to change the occurrence or recurrence of elder abuse is uncertain. Similarly, a study a with low risk of bias (Cooper 2015) aimed at promoting the mental health of carers was successful at improving certain mental health measures, however it found no difference in the 'harder' outcome of reporting behaviours of elder abuse.

Other interventions identified in this review sought to intervene on victims or perpetrators with the intention to prevent the recurrence of abuse. While there was some evidence that these interventions may have some effect on more distal outcomes (e.g. attitudes and coping), there is no evidence to show an effect on the occurrence of elder abuse.

\section{Educational Interventions}

Educational interventions provided the largest body of evidence in this review. Most educational interventions focused primarily on healthcare professionals. There is some limited evidence to suggest that educational interventions improve knowledge and attitudes towards elder abuse among healthcare professionals. There is no evidence to suggest if educational interventions prevent elder abuse or reduce recurrent elder abuse or other related outcomes. There is, however, emerging evidence (Teresi 2013) that education interventions for healthcare professionals might improve detection and management of elder abuse.

There is very little detailed information available about the educational intervention programme that could be a useful guide for future curriculum development. There are significant variations in the methods of delivery, frequency and intensity of the educational programmes used in the included studies. Didactic, face-to-face sessions were the most common method of information dissemination which were conducted as a one-off session except for Teresi 2013 and Hsieh 2009. Only one study compared the effectiveness of the methods of information delivery (Richardson 2002). The findings from that study suggests that dissemination of printed information is less effective compared to face-to-face didactic sessions. An observation that we made in this review was of the lack of underlying theoretical basis to inform these programmes. Outcome measures were different across studies, particularly in relation to the tools used and duration of the measurement. With such variations, it is difficult to draw any useful comparisons. Most studies did not provide enough information on the development and process of these intervention programs to be replicable in other populations or settings.

\section{Programme to reduce factors influencing elder abuse}

There is no strong evidence to suggest that programmes specifically targeting risk factors on elder abuse actually prevent or reduce elderly abuse. The only randomised controlled trial included in this review that examined this type of programme found no significant difference in the reporting of abusive behaviour among carers in the intervention group compared to those in the control group (Cooper 2015).

Programme to increase detection rate for prevention of elder abuse 
We did not have enough evidence to draw a firm conclusion about the effectiveness of programmes specifically aiming to increase detection through interventions such as home visits, home-based geriatric assessment and helplines. Neither is there information about whether they are useful to reduce occurrence or recurrence of abuse. In one study (Bartels 2005), which compared clinicians who performed usual care with clinicians in the intervention group using a new integrated system of clinical assessment, and decision support for elderly with mental illness. The clinicians in the intervention group were more likely to screen for elder safety, neglect and abuse during the 12-month period than the clinicians in the control (usual care) group.

\section{Programmes targeted to victims}

Findings from this review suggest that there is insufficient trustworthy evidence to identify which type of victims' programmes are most effective and under what circumstances. There is an indication that there may be negative, harmful effects associated with these programmes where abuse was reported higher among the elderly who received home visits than those in the control group (Davis 2001). The study authors hypothesised that it was possible that the abusers of the elderly became angered by attempts to intervene. There is also the possibility that the elderly who received the intervention were more likely to report new instances of abuse to police and research personnel during or after the intervention.

On the other hand, Brownell 2006 did not find any significant change in outcomes among older women who were victims of mistreatment receiving psycho-social support. The insignificant outcomes may be largely attributed to participants already receiving services from other aging services providers prior to the study, or due to the ineffectiveness of the programmes or due to programme implementation factors (not reported in the studies).

\section{Rehabilitation programmes for perpetrators}

There is an absence of evidence to support any particular intervention related to elder abuse that targets perpetrators.

\section{Overall completeness and applicability of evidence}

The logic model, published in the protocol for this review, nominated seven avenues through which interventions may act (education, reducing influential risk factors, legislation, policies for the elderly, programmes to increase detection, programmes targeted to victims, rehabilitation programs). Three of the seven studies included in this review were of educational interventions directed at health professionals or carers, with another aiming to reduce risk factors of carers, one to increase detection, two of programs targeting victims (one primarily around education and one utilising a psycho-social support group and providing educational material), and one was a programme targeting perpetrators. Many of these studies were of high risk of bias or were very small and thus lacked trustworthiness. There are therefore considerable gaps in the available evidence and in our understanding of effective mechanisms to reduce the incidence of elder abuse.

Analysing and applying the body of evidence is complicated by the variety and reliability of measures employed in the trials. Measuring outcomes of elder abuse is complicated by the complex causes and manifestations of elder abuse, as well as the distal and longterm nature of many of the interventions conflicting with the pragmatic realities of trials. Further, depending on the context, instances of elder abuse may be rare therefore requiring a large sample size to discern any meaningful difference. Investigators may therefore adopt proxy or intermediate measures that reflect the cascade of actions that the intervention being evaluated is intended to produce. For example, an intervention may be designed to increase knowledge, then behaviour change, improve assessment and reporting, and eventually prevent instances of elder abuse, or improving detection. If hard outcomes are unable to be used then there is a need for validated, relevant and meaningful proxy outcomes. The absence of these inevitably leads to questions about how much confidence can be placed in the link between proxy measures and abuse.

There is also significant need for further development and evaluation of interventions, as well as the need to explore efficacy in different settings. All studies were conducted in high-income countries, in three western countries and one Asian country. Hsieh 2009 investigated an educational intervention on nursing home caregivers in Taiwan. The literature therefore provides little guidance as to the likely effectiveness of interventions in different settings, particularly in middle- and lower-income countries where there are often different expectations and practices in caring for the elderly, and therefore likely different pathways to abuse. The limited number of settings explored in the included data, therefore further limits the applicability of the study findings.

\section{Quality of the evidence}

Two of the studies were non-randomised (Bartels 2005; Hsieh 2009), which was particularly problematic in that other aspects of study design were often not strongly conducted. Several studies had very small sample sizes and there were further issues in relation to the validation of measurements and outcomes employed. Many of the studies were at high risk of bias or unclear risk of bias for most domains, so we down-graded the quality of the evidence to low or very low.

\section{Potential biases in the review process}

There were several potential biases that we encountered during identification of relevant studies in the review process. Firstly, the multidisciplinary nature of this topic and the heterogeneity of the interventions prompted us to adopt a broad search strategy approach. The topic crosses multiple disciplines such as health, medicine, social sciences, law, and policies. During the search process, we found there is a lack of standard and clear terminology used within this topic due to the range of disciplines, countries and the type of abuse covered in this review. An extensive list of terms and synonyms were utilised to capture the concept of "abuse" itself, ranging from abuse, maltreatment, mistreatment, assault, neglect and so on. A similar issue was identified for the concept of 'elder' and various interventions where many terms and synonyms were used in addition to employing truncation and adjacency operators during the search process to minimise any potential risk of missing any relevant studies. However, a broad search approach drew a large number of irrelevant literature as evidence in Figure 2. In addition, few bibliographic databases allowed limited terms to be searched, hence presenting the risk that relevant studies could be missed in this review. The databases searches tended to duplicate each other. 
This review has a strict inclusion criteria, primarily including interrupted time series (ITS), controlled before-and-after (CBA) and randomised controlled trial (RCT) study designs that have at least 12 weeks of follow-up period. During the screening process, we found a number of relevant elder abuse interventions, but these were mainly descriptive, observational studies and case studies. In cases where the study designs fitted the inclusion criteria, there were studies which had measurement time points limited to pre- and one post measurement, or no comparison groups. Three excluded studies originally appeared as ITS design ( Cooper 2012; Nusbaum 2007; Reay 2002), but a closer examination revealed that there was inadequate pre-intervention measurements of outcomes. We argue that the strict inclusion criteria is necessary given that it is absolutely vital to identify methodologically rigorous studies that can provide evidence of sustainable outcomes.

\section{Agreements and disagreements with other studies or reviews}

This review concurs with findings from four recent reviews conducted ( Alt 2011; Daly 2011; Ploeg 2009; WHO 2011) that there is insufficient evidence on elder abuse interventions to demonstrate prevention or reduction of elder abuse. Among these interventions, in particular, no low risk of bias studies on provision adult protective services, emergency shelters, legislation support, public information or educational campaigns or intergenerational programmes, restraint-reduction programmes and helplines that could be included in this review. Although we identified a number of interventions implemented in various countries, three major issues identified were: 1) interventions are embedded within a larger study, not specifically address to elder abuse, 2) the scarcity of a comprehensive evaluation on the effectiveness of these programme (e.g. lack of multiple time point measurements or long-term follow-up), and 3) intervention not measuring abuserelated outcomes and its cost effectiveness. This research, as shown in the Characteristics of excluded studies, concurs with reviews such as Alt 2011 that a large proportion of the literature on elder abuse describes programs for elder abuse which are often brief, without a comparison, and in many cases, only report post-intervention descriptions of satisfaction with a program. Although these publications are useful to describe the processes and acceptability of the interventions, they fail to meet the criteria to establish a causal relationship (Schünemann 2011a) for abuse prevention that policy makers and health professionals can use. These findings are frustrating as much earlier, the Wilson 2003 review identified few evaluations of interventions to end or reduce elder abuse, with the majority of the literature at high risk of bias.

\section{AUTHORS' CONCLUSIONS}

\section{Implications for practice}

The issues discussed previously limit the implications that can be drawn from this body of evidence in the adoption of strategies to decrease elder abuse. Broadly, the current available evidence suggests that it is uncertain whether targeted educational interventions improve relevant knowledge of health professionals and caregivers. A blended intervention of education and support aimed at victims also may improve rates of reporting, although some of the studies also suggest that these results may have uncertain outcomes on the reporting or detection of abuse. This review also presents evidence of the potential effectiveness

Interventions for preventing abuse in the elderly (Review)

Copyright (c) 2016 The Cochrane Collaboration. Published by John Wiley \& Sons, Ltd. of service planning interventions at probably improving the assessment and documentation of related domains.

The variety in settings and study trustworthiness limits the inferences that can be drawn in applying these findings to practice. However, recognising that caregivers and health providers currently implement some of the strategies identified in this review, it is important that evaluation components, both qualitative and quantitative, be undertaken during service delivery to inform future research and interpretation.

\section{Implications for research}

There is still much to be done in this field as very few studies have been undertaken which have the ability to identify a causal relationship between the intervention and the outcomes of abuse (occurrence or recurrence). There is likely a need to better understand the mechanism and circumstances that increase the likelihood of elder abuse across different settings. The development and evaluation approaches to try and understand effectiveness in these contexts would then provide useful guidance.

The main implication of this review is that research is needed to resolve uncertainties on the effectiveness of different intervention programmes utilised to reduce and/or prevent elder abuse in organisational/institutional and community settings. The evidence in this review was from the United States (four studies), United Kingdom (two studies), and the remaining study was from Taiwan. Evidence from different parts of the world, especially from the lowand middle-income countries is lacking. Public health practitioners may wish to resolve this uncertainty by encouraging more research, and for such research to be done in developing countries as well. In particular, well-constructed, high-quality research- even if nonrandomised, especially in areas of policy and legislation is also necessary to further understand the potential utility of these levers.

We recognised that methodologically strong research and/or comprehensive national/state level programmes are currently ongoing (e.g. Loh 2015), whereby the results would significantly contribute to the existing available evidence on prevention and reduction of elder abuse.

Although we retrieved a number of studies on interventions targeting possible risk factors related to elder maltreatment (e.g. dementia, caregiver burden, disability), these interventions were neither targeted to address elder abuse or measured any outcomes related to elder abuse. Hence future research should address this as many of these risk factors can be useful indicators for elder abuse primary prevention (i.e. before abuse occurs).

\section{ACKNOWLEDGEMENTS}

The authors wish to acknowledge the University of Malaya Research Grant (UMGC001B-14HTM) for funding this study. Noran Naqiah Hairi's work was supported by the University of Malaya/Ministry of Higher Education (UM/MOHE) High Impact Research Grant UM.C/625/1/HIR/MOHE/ASH/02. University of Malaya provided visiting professor and research funding travel grant for Philip Baker and Daniel Francis. Funding was also received from Queensland University of Technology's Institute for Health and Biomedical Inovation for funding of literature searching.

The authors would like to thank the members of the Review Advisory Group; Associate Professor Dr Susan Kurrle from 
University of Sydney, Australia; Professor Brian J Taylor from University of Ulster, Northern Ireland; Paula M Mixson of the National APS (Adult Protective Services) Association and the National Committee for the Prevention of Elder Abuse; Professor Michael Dunne, Queensland University of Technology, Australia; and Mrs Nell McFadden, Joseph Rowntree Foundation for their advice and contribution of; Ruth Turley for providing assistance on searching strategies in the protocol development; Oscar Ovid Trespalcios, Catherine Haden, Cameron Rutter, Shameny Chandra and Pathma Devi Veerasingam for assistance in searching and assessing literature; Anne Chu for assessing and study retrieval of Chinese literature.Cochrane Public Health Information Specialist, Patrick Condron, provided advice regarding searching. 


\section{R E F E R E N C E S}

\section{References to studies included in this review}

Bartels 2005 \{published data only\}

Bartels SJ, Miles KM, Van Citters AD, Forester BP, Cohen MJ, Xie H. Improving mental health assessment and service planning practices for older adults: a controlled comparison study. Mental Health Services Research 2005 Dec;7(4):213-23.

Brownell 2006 \{published data only\}

Brownell P, Heiser D. Psycho-educational support groups for older women victims of family mistreatment: a pilot study. Journal of Gerontological Social Work 2006;46(3-4):145-60.

\section{Cooper 2015 \{published data only\}}

* Cooper C, Barber J, Griffin M, Rapaport P, Livingston G. Effectiveness of START psychological intervention in reducing abuse by dementia family carers: randomized controlled trial. International Psychogeriatrics 2015; Vol. Epub ahead of print:1-7.

Livingston G, Barber J, Rapaport P, Knapp M, Griffin M, King D, et al. Clinical effectiveness of a manual based coping strategy programme (START, STrAtegies for RelaTives) in promoting the mental health of carers of family members with dementia: pragmatic randomised controlled trial. BMJ 2013;347:f6276.

\section{Davis 2001 \{published data only\}}

* Davis RC, Medina J, Avitabile N. Reducing repeat incidents of elder abuse: Results of a randomized experiment: Final report. Reducing repeat incidents of elder abuse: Results of a randomized experiment: Final report. U.S. Department of Justice: Office of Justice Program, 2001. [Document no 189086]

Davis RC, Medina-Ariza J. Results from an elder abuse prevention experiment in New York City. Available from https:// www.ncjrs.gov/pdffiles1/nij/188675.pdf 2001.

\section{Hsieh 2009 \{published data only\}}

Hsieh HF, Wang JJ, Yen M, Liu TT. Educational support group in changing caregivers' psychological elder abuse behavior toward caring for institutionalized elders. Advances in Health Sciences Education 2009;14(3):377-86.

\section{Richardson 2002 \{published data only\}}

Richardson B, Kitchen G, Livingston G. The effect of education on knowledge and management of elder abuse: a randomized controlled trial. Age and Ageing 2002;31(5):335.

\section{Teresi 2013 \{published data only\}}

Teresi JA, Ramirez M, Ellis J, Silver S, Boratgis G, Kong J, et al. A staff intervention targeting resident-to-resident elder mistreatment (R-REM) in long-term care increased staff knowledge, recognition and reporting: results from a cluster randomized trial. International Journal of Nursing Studies 2013 May;50(5):644-56.

\section{References to studies excluded from this review}

Acierno 2004 \{published data only\}

Acierno R, Rheingold AA, Resnick HS, Stark-Riemer W. Preliminary evaluation of a video-based intervention for older adult victims of violence. Journal Trauma Stress 2004;17(6):535-41.

\section{ACT government 2006 \{published data only\}}

ACT Government. ACT elder abuse policy framework: assisting ACT Government agencies in developing policy and service responses. Canberra: ACT Government, 2006. [http:// www.dhcs.act.gov.au/wac/ageing/files/pdf/ACT_Elder_Abuse Policy_Framework1.jt1.pdf]

\section{Action on Elder Abuse 1995 \{published data only\}}

Action on Elder Abuse (AEA). Adult protection data collection and reporting requirements: conclusions and recommendations from a two year study into adult protection recording systems in England. London: Action On Elder Abuse, 2006. [http://www.elderabuse.org.uk/Documents/ AEA\%20Report\%20-\%20Data\%20Monitoring\%20-\%20DH \%20Monitoring\%20Project.pdf]

\section{Action on Elder Abuse 2006 \{published data only\}}

Action on Elder Abuse (AEA). Adult protection data collection and reporting requirements: conclusions and recommendations from a two year study into adult protection recording systems in England. London: Action On Elder Abuse, 2006. [http://www.elderabuse.org.uk/Documents/ AEA\%20Report\%20-\%20Data\%20Monitoring\%20-\%20DH $\% 20$ Monitoring\%20Project.pdf]

Aday 1991 \{published data only\}

Aday RH, Sims CR, Evans E. Youth's attitudes toward the elderly: the impact of intergenerational partners. Journal of Applied Gerontology 1991;10(3):372-84

Aday 1993 \{published data only\}

Aday RH, McDuffie W, Sims CR. Impact of an intergenerational program on black adolescents' attitudes towards the elderly. Educational Gerontology 1993;19(7):663-73.

Age Concern New Zealand 2005 \{published data only\} Age Concern New Zealand. Age Concern Elder Abuse and Neglect Prevention Services - An Analysis of Referrals for the Period: 1 July 2002 to 30 June 2004. Wellington, NZ.: Age Concern New Zealand, November 2005. [http:// ageconcern.org.nz/files/file/EANP_final.pdf ]

Anetzberger 1993 \{published data only\}

Anetzberger GJ. Elder abuse programming among geriatric education centers. Journal of Elder Abuse \& Neglect 1993;5(3):69-85.

\section{Anetzberger 2000b \{published data only\}}

Anetzberger GJ, Alfonso HI. Mortgage Fraud Prevention Program: Volunteer Legal Services Program of the Bar 
Association of San Francisco. Journal of Elder Abuse \& Neglect 2000;12(2):75-8.

\section{Anetzberger 2010 \{published data only\}}

Anetzberger GJ, Balaswamy S. Elder abuse awareness and action: the role of state summits. Journal of Elder Abuse \& Neglect 2010;22(1-2):180-90.

\section{Anetzerberger 2000a \{published data only\}}

Anetzberger GJ, Palmisano BR, Sanders M, Bass D, Dayton C, Eckert $S$, et al. A model intervention for elder abuse and dementia. Gerontologist 2000;40(4):492-7.

\section{Antonio 2009 \{published data only\}}

Antonio PS, Simon-Rusinowitz L, Loughlin D, Eckert JK, Mahoney KJ, Ruben KAD. Lessons From the Arkansas Cash and Counseling program: how the experiences of diverse older consumers and their caregivers address family policy concerns. Journal of Aging \& Social Policy 2009;22(1):1-17.

\section{Ash 2013 \{published data only\}}

Ash A. A cognitive mask? Camouflaging dilemmas in street-level policy implementation to safeguard older people from abuse. British Journal of Social Work 2013;43(1):99-115. [DOI: 10.1093/ bjsw/bcr161]

\section{Austin 2005 \{published data only\}}

Austin CD, Descamp E, Flux D, McClelland R, Sieppert J. Community development with older adults in their neighborhoods: the elderly friendly communities program. Families in Society: The Journal of Contemporary Social Services 2005;86(3):401-10.

\section{Belle 2006 \{published data only\}}

Belle SH, Burgio L, Burns R, Coon D, Czaja SJ, GallagherThompson $D$, et al. Enhancing the quality of life of dementia caregivers from different ethnic or racial groups: a randomized, controlled trial. Annals of internal medicine 2006;145(10):727-38.

\section{Ben Natan 2013 \{published data only\}}

Ben Natan M, Tabak N. Combating the maltreatment of older persons by staff in long-term care nursing homes: legal aspects. Medicine and Law 2013;32(1):53-64.

\section{Bennett 1993 \{published data only\}}

Bennett G, Kingston P. Elder Abuse: Concepts, Theories and Interventions. 1st Edition. Chapman \& Hall, New York, NY; and Distributed in the USA and Canada by Singular Pub. Group, San Diego, CADistributed in the USA and Canada by Singular Pub. Group, San Diego, CA, 1993.

\section{Bennett 2000 \{published data only\}}

Bennett GCJ, Ginny J, Zee A. Listening is not enough: An analysis of calls to the Elder Abuse Response. Journal of Adult Protection 2000;2(1):6-20.

\section{Blakely 2001 \{published data only\}}

Blakely BE, Dolon R. Another look at the helpfulness of occupational groups in the discovery of elder abuse and neglect. Journal of Elder Abuse \& Neglect 2001;13(3):1-23.

\section{Bomba 2006 \{published data only\}}

Bomba PA. Use of a single page elder abuse assessment and management tool: a practical clinician's approach to identifying elder mistreatment. Journal of Gerontological Social Work 2006;46(3-4):103-22.

\section{Bowland 2012 \{published data only\}}

Bowland S, Edmond T, Fallot R. Evaluation of a spiritually focused intervention with older trauma survivors. Social Work 2012;57(1):73-82.

\section{Braun 1997 \{published data only\}}

Braun KL, Suzuki KM, Cusick CE, Howard-Carhart K. Developing and testing training materials on elder abuse and neglect for nurse aids. Journal of Elder Abuse \& Neglect 1997;9(1):1-15.

\section{Braun 2004 \{published data only\}}

Braun K, Sorrell R, Fujita R, Kreider D. Evaluating REACH: an abuse and neglect prevention program. Gerontologist 2004;44(1):436.

\section{Breen 2009 \{published data only\}}

Breen GM, Matusitz J, Wan TT. The use of public policy analysis to enhance the Nursing Home Reform Act of 1987. Social Work in Health Care 2009;48(5):505-18. [DOI: 10.1080/00981380902734630]

\section{Brown 1992 \{published data only\}}

Brown DS, Gardner DL, Perritt L, Kelly DG. Improvement in attitudes toward the elderly following traditional and geriatric mock clinics for physical therapy students. Physical Therapy 1992;72(4):251-7.

\section{Brown 1998 \{published data only\}}

Brown $\mathrm{H}$, Stein J. Implementing adult protection policies in Kent and East Sussex. Journal of Social Policy 1998;27(3):371-96.

\section{Brown 2004 \{published data only\}}

Brown K, Streubert GE, Burgess AW. Effectively detect and manage elder abuse. Nurse Practitioner 2004;29(8):22-7.

\section{Brownell 2003 \{published data only\}}

Brownell P, Wolden A. Elder abuse intervention strategies: social service or criminal justice?. Journal of Gerontological Social Work 2003;40:83-100.

\section{Butler 2008 \{published data only\}}

Butler FR, Baghi H. Using the internet to facilitate positive attitudes of college students toward aging and working with older adults. Journal of Intergenerational Relationships 2008;6(2):175-89.

\section{Cambridge 2006 \{published data only\}}

Cambridge P, Parkes T. The tension between mainstream competence and specialization in adult protection: an evaluation of the role of the adult protection co-ordinator. British Journal of Social Work 2006;36(2):299-321.

\section{Cambridge 2011 \{published data only\}}

Cambridge P, Beadle-Brown J, Milne A, Mansell J, Whelton B. Adult protection: The processes and outcomes of adult 
protection referrals in two English local authorities. Journal of Social Work 2011;11(3):247-67.

Chaffin 2002 \{published data only\}

Chaffin J, Richter P. Making prevention of abuse and neglect through dental awareness green. Military Medicine 2002;167(11):920-2.

\section{Chang 2003 \{published data only\}}

Chang VN, Greene R. Study of service delivery by community mental health centers as perceived by adult protective services investigators. Journal of Elder Abuse \& Neglect 2003;13(3):25-42.

\section{Clancy 2011 \{published data only\}}

Clancy M, McDaid B, O'Neill D, O'Brien JG. National profiling of elder abuse referrals. Age and Ageing 2011;40(3):346-51.

\section{Cook-Daniels 2004 \{published data only\}}

Cook-Daniels L. Training of adult protective services workers: a survey report. Victimization of the Elderly and Disabled 2004;7(3):37.

\section{Coon 2003 \{published data only\}}

Coon DW, Thompson L, Steffen A, Sorocco K, GallagherThompson D. Anger and depression management: psychoeducational skill training interventions for women caregivers of a relative with dementia. Gerontologist 2003;43(5):678-89.

\section{Cooper 2012 \{published data only\}}

Cooper C, Huzzey L, Livingston G. The effect of an educational intervention on junior doctors' knowledge and practice in detecting and managing elder abuse. International Psychogeriatrics 2012 Sep;24(9):1447-53.

\section{Cummings 2002 \{published data only\}}

Cummings SM, Williams MM, Ellis RA. Impact of an intergenerational program on 4th graders' attitudes toward elders and school behaviors. Journal of Human Behavior in the Social Environment 2002;6(3):91-107.

\section{Cupitt 1997 \{published data only\}}

Cupitt M. Identifying and addressing the issues of elder abuse: a rural perspective. Journal of Elder Abuse \& Neglect 1997;8(4):21-30.

\section{Daly 2003 \{published data only\}}

Daly JA, Jogerst GJ, Brinig MF. Mandatory reporting: Relationship of APS statute language on state reported elder abuse. Journal of Elder Abuse \& Neglect 2003;15(2):1-21.

\section{Daly 2011a \{published data only\}}

Daly JM. Domestic and institutional elder abuse legislation. Nursing Clinics of North America 2011;46(4):477-84, vii.

\section{Daniels 1989 \{published data only\}}

Daniels RS, Baumhover LA, Clark-Daniels CL. Physicians' mandatory reporting of elder abuse. Gerontologist 1989;29(3):321-7.

\section{Dauenhauer 2007 \{published data only\}}

Dauenhauer JA, Cattat Mayer K, Mason A. Evaluation of adult protective services: perspectives of community professionals. Journal of Elder Abuse \& Neglect 2007;19(3-4):41-57.

Davies 2011 \{published data only\}

Davies ML. Detecting and preventing financial abuse of older adults: Examining decision making by health, social care and banking professionals. Diss. Brunel University School of Health Sciences and Social Care PhD Theses. Brunel University School of Health Sciences and Social Care PhD Theses, 2011.

Day 2010 \{published data only\}

Day MR, Bantry-White E, Glavin P. Protection of vulnerable adults: an interdisciplinary workshop. Community Practitioner : the journal of the Community Practitioners' \& Health Visitors' Association 2010;83(9):29-32.

Dellmann-Jenkins 1991 \{published data only\} Dellmann-Jenkins M, Lambert D, Fruit D. Fostering preschoolers' prosocial behaviors toward the elderly: the effect of an intergenerational program. Educational Gerontology 1991;17(1):21-32

Desy 2008 \{published data only\}

Desy PM, Prohaska TR. The Geriatric Emergency Nursing Education (GENE) course: an evaluation. Journal of Emergency Nursing 2008;34(5):396-402.

Dillenburger 2008 \{published data only\}

Dillenburger K, Fargas M, Akhonzada R. Evidence- based practice: an exploration of the effectiveness of voluntary sector services for victims of community violence. British Journal of Social Work 2008;38(8):1630-47.

Dolon 1989 \{published data only\}

Dolon R, Blakely B. Elder abuse and neglect: A study of adult protective service workers in United States. Journal of Elder Abuse \& Neglect 1989;1(3):31-50.

Dorfman 2003 \{published data only\}

Dorfman LT, Murty S, Ingram JG, Evans RJ. Incorporating intergenerational service-learning into an introductory gerontology course. Journal of Gerontological Social Work 2003;39(1-2):219-40

\section{Drossel 2011 \{published data only\}}

Drossel C, Fisher JE, Mercer V. A DBT Skills training group for family caregivers of persons with dementia. Behavior Therapy 2011;42(1):109-19.

Eisdorfer 2003 \{published data only\}

Eisdorfer C, Czaja SJ, Loewenstein DA, Rubert MP, Argüelles S, Mitrani VB, et al. The effect of a family therapy and technologybased intervention on caregiver depression. Gerontologist 2003;43(4):521-31.

Ejaz 2001 \{published data only\}

Ejaz FK, Bass DM, Anetzberger GJ, Nagpaul K. Evaluating the Ohio elder abuse and domestic violence in late life screening 
tools and referral protocols. Journal of Elder Abuse \& Neglect 2001;13(2):39-57.

\section{Elliott 2010 \{published data only\}}

Elliott AF, Burgio LD, Decoster J. Enhancing caregiver health: findings from the resources for enhancing Alzheimer's caregiver health II intervention. Journal of the American Geriatric Society 2010;58(1):30-7.

\section{Ernst 2012 \{published data only\}}

Ernst JS, Smith CA. Assessment in Adult Protective Services: do multidisciplinary teams make a difference?. Journal of Gerontological Social Work 2012;55(1):21-38.

Everson 1996 \{published data only\}

Everson NC. Teaching banks how to protect their older customers. Aging 1996;0(367):80-3.

\section{Feldt 1992 \{published data only\}}

Feldt KS, Ryden MB. Aggressive behavior: educating nursing assistants. Journal of Gerontological Nursing 1992;18(5):3-12.

Fiegener 1989 \{published data only\}

Fiegener JJ, Fiegener M, Meszaros J. Policy Implications of a Statewide Survey on Elder Abuse. Journal of Elder Abuse \& Neglect 1989;1(2):39-58.

\section{Filinson 1993 \{published data only\}}

Filinson R. An evaluation of a program of volunteer advocates for elder abuse victims. Journal of Elder Abuse \& Neglect 1993;5(1):77-93.

\section{Filinson 2001 \{published data only\}}

Filinson R. Evaluation of the impact of a volunteer Ombudsman Program: The Rhode Island experience. Journal of Elder Abuse \& Neglect 2001;13(4):1-19.

\section{Filinson 2006 \{published data only\}}

Filinson R. "No secrets" and beyond: recent elder abuse policy in England. Journal of Elder Abuse \& Neglect 2006;18(1):1-18.

\section{Finkel 2007 \{published data only\}}

Finkel S, Czaja SJ, Schulz R, Martinovich Z, Harris C, Pezzuto D. E-care: A telecommunications technology intervention for family caregivers of dementia patients. American Journal of Geriatric Psychiatry 2007;15(5):443-8.

Fisher 2003 \{published data only\}

Fisher BS, Zink T, Pabst S, Regan S, Rinto B. Services and programming for older abused women: The Ohio experience. Journal of Elder Abuse \& Neglect 2003;15(2):67-83.

Fisher 2003a \{published data only\} Fisher R, D'Arpino M, Dion T, Glazier S, Moghabghab R, Elizabeth $\mathrm{O}$, et al. Elder abuse workshop for healthcare providers. Geriatrics and Aging 2003;6(6):61-3.

\section{Foelker 1990 \{published data only\}}

Foelker GA. A community response to elder abuse. Gerontologist 1990;30(4):560-2.
Fox 1996 \{published data only\}

Fox SD, Wold JE. Baccalaureate student gerontological nursing experiences: raising consciousness levels and affecting attitudes. Journal of Nursing Education 1996;35(8):348-55.

Fujiwara 2006 \{published data only\}

Fujiwara Y, Nishi M, Watanabe N, Lee S, Inoue K, Yoshida H, et al. An intergenerational health promotion program involving older adults in urban areas. "Research of Productivity by Intergenerational Sympathy (REPRINTS)": first-year experience and short-term effects. Nihon Koshu Eisei Zasshi 2006;53:702-14.

Fulmer 1985 \{published data only\}

Fulmer T, Wetle T. [Protocols for the assessment of elder abuse. In: Paper presented at the 38th Annual Scientific Meeting of the Gerontological Society]. Paper presented at the 38th Annual Scientific Meeting of the Gerontological Society, November 22-26. New Orleans, LA, 1985:12p.

\section{Gallagher-Thompson 2007 \{published data only\}}

Gallagher-Thompson D, Gray HL, Tang PC, Pu CY, Leung LY, Wang PC, et al. Impact of in-home behavioral management versus telephone support to reduce depressive symptoms and perceived stress in Chinese caregivers: results of a pilot study. American Journal of Geriatric Psychiatry 2007 May;15(5):425-34.

\section{Gassoumis 2015 \{published data only\}}

Gassoumis ZD, Navarro AE, Wilber KH. Protecting victims of elder financial exploitation: The role of an Elder Abuse Forensic Center in referring victims for conservatorship. Aging \& Mental Health 2015;19(9):790-8.

\section{Georgia 1999 \{published data only\}}

Georgia JA, Dyer CB, Barth J, Portal B, Hyman DJ, Pavlik VN, et al. Case series of abused or neglected elders treated by an interdisciplinary geriatric team. Journal of Elder Abuse \& Neglect 1999;10(3-4):131-9.

\section{Georgia 1999a \{published data only\}}

Georgia JA, Yamada Y. A telephone counseling program for elder abuse in Japan. Journal of Elder Abuse \& Neglect 1999;11(1):105-12.

\section{Gillum 2009 \{published data only\}}

Gillum TL, Sun CJ, Woods AB. Can a health clinic-based intervention increase safety in abused women? Results from a pilot study. Journal of Women's Health 2009;18(8):1259-64.

\section{Gironda 2010 \{published data only\}}

Gironda MW, Lefever K, Delagrammatikas L, Nerenberg L, Roth $\mathrm{R}$, Chen EA, et al. Education and training of mandated reporters: innovative models, overcoming challenges, and lessons learned. Journal of Elder Abuse \& Neglect 2010;22(3-4):340-64.

\section{Gittler 2008 \{published data only\}}

Gittler J. Governmental efforts to improve quality of care for nursing home residents and to protect them from mistreatment: a survey of federal and state laws. Research in Gerontological Nursing 2008;1(4):264-84. [DOI: 10.3928/19404921-20081001-08] 
Gold 1989 \{published data only\}

Gold DT, Gwyther LP. The prevention of elder abuse: an educational model. Family Relations 1989;38(1):8-14.

\section{Goldberg 2001 \{published data only\}}

Goldberg E. An evaluation of the health care fraud education project [3003352]. United States - Pennsylvania: Widener University 2001.

\section{Goodrich 1997 \{published data only\}}

Goodrich CS. Results of a national survey of state protective services programs: assessing risk and defining victim outcomes. Journal of Elder Abuse \& Neglect 1997;9(1):69-86.

\section{Goodridge 1997 \{published data only\}}

Goodridge D, Johnston P, Thomson M. Impact of a nursing assistant training program on job performance, attitudes, and relationships with residents. Educational Gerontology 1997;23(1):37-51.

\section{Gorgen 2005 \{published data only\}}

Gorgen T, Nagele B. Domestic elder abuse and neglect Conclusions from the evaluation of a model project. Zeitschrift fur Gerontologie und Geriatrie 2005;38(1):4-9.

\section{Griffith 2009 \{published data only\}}

Griffith R, Tengnah C. The protection of vulnerable adults. British Journal of Community Nursing 2009;14(6):262-6.

\section{Groh 2011 \{published data only\}}

Groh A, Linden R. Addressing elder abuse: the Waterloo restorative justice approach to elder abuse project. Journal of Elder Abuse \& Neglect 2011;23(2):127-46.

\section{Hagen 1995 \{published data only\}}

Hagen BF, Sayers D. When caring leaves bruises: The effects of staff education on resident aggression. Journal of Gerontological Nursing 1995;21:7-16.

\section{Hannon 2008 \{published data only\}}

Hannon PO, Gueldner SH. The impact of short-term quality intergenerational contact on children's attitudes toward older adults. Journal of Intergenerational Relationships 2008;5(4):59-76

\section{Hanratty 2011 \{published data only\}}

Hanratty M. Impacts of Heading Home Hennepin's Housing First programs for long-term homeless adults. Hous Policy Debate 2011;21(3):405-19.

\section{Harmer-Beem 2005 \{published data only\}}

Harmer-Beem M. The perceived likelihood of dental hygienists to report abuse before and after a training program. Journal of Dental Hygiene 2005;79(1):1-12.

\section{Harries 2014 \{published data only\}}

Harries P, Davies M, Gilhooly K, Gilhooly M, Tomlinson C. Educating novice practitioners to detect elder financial abuse: a randomised controlled trial. BMC Medical Education 2014;14:21.
Harry 2000 \{published data only\}

Harry D, Bill H. Reviewing practice and procedures in adult protection in the Southern Health and Social Services Board. Journal of Adult Protection 2000;2(2):41-6.

\section{Hawes 2010 \{published data only\}}

Hawes C, Kimbell A-M. Catherine Hawes and Anne-Marie Kimbell. Detecting, Addressing and Preventing Elder Abuse In Residential Care Facilities. Rockville, United States: National Institute of Justice/NCJRS, 2009. [https://www.ncjrs.gov/ pdffiles1/nij/grants/229299.pdf]

Heath 2002 \{published data only\} Heath JM, Dyer CB, Kerzner LJ, Mosqueda L, Murphy C. Four models of medical education about elder mistreatment. Academic Medicine 2002 Nov;77(11):1101-6.

Heath 2005 \{published data only\}

Heath JM, Kobylarz FA, Brown M, Castano S. Interventions from home-based geriatric assessments of adult protective service clients suffering elder mistreatment. Journal of the American Geriatrics Society 2005;53(9):1538-42.

Hermoso 2006 \{published data only\} Hermoso J, Rosen AL, Overly L, Tompkins CJ. Increasing aging and advocacy competency: the intergenerational advocacy pilot project. Journal of Gerontological Social Work 2006;48:179-92.

Hill 1986 \{published data only\}

Hill B, Hwalek M, Stahl C. The Illinois elder abuse demonstration projects- monitoring program development. Gerontologist 1986;26:A22

\section{Hodge 1998 \{published data only\}}

Hodge PD. National law enforcement programs to prevent, detect, investigate, and prosecute elder abuse and neglect in health care facilities. Journal of Elder Abuse \& Neglect 1998;9(4):23-41.

Holkup 2007 \{published data only\} Holkup PA, Salois EM, Tripp-Reimer T, Weinert C. Drawing on wisdom from the past: an elder abuse intervention with tribal communities. Gerontologist 2007;47(2):248-54.

Hudson 1993 \{published data only\}

Hudson B. Ensuring an abuse-free environment. Journal of Elder Abuse \& Neglect 1993;4(4):25-36.

Hughes 1995 \{published data only\} Hughes M. Abuse of older people: a network approach to service provider training. Australian Social Work 1995;48(4):21-7.

Hutchison 1993 \{published data only\}

Hutchison RR, Quartaro EG. Training imperatives for volunteers caring for high-risk, vulnerable populations. Journal of Community Health Nursing 1993;10(2):87-96. 
Huynh-Hohnbaum 2008 \{published data only\}

Huynh-Hohnbaum AL, Villa VM, Aranda MP, Lambrinos J. Evaluating a multicomponent caregiver intervention. Home Health Care Services Quarterly 2008;27(4):299-325.

\section{Hwalek 1988 \{published data only\}}

Hwalek, M. Elder Abuse: The Illinois Department on Aging's Final Report on the Elder Abuse Demonstration Program Act. Ilinois Department on Aging, 1998. [https://www.ncjrs.gov/ pdffiles1/Digitization/113329NCJRS.pdf]

\section{Iris 1990 \{published data only\}}

Iris MA. Uses of guardianship as a protective intervention for frail, older adults. Journal of Elder Abuse \& Neglect 1990;2(3-4):57-72.

\section{Jackson 2012 \{published data only\}}

Jackson SL, Hafemeister TL. APS investigation across four types of elder maltreatment. Journal of Adult Protection 2012;14(2):82-92.

\section{Jackson 2013 \{published data only\}}

Jackson SL, Hafemeister TL. How do abused elderly persons and their adult protective services caseworkers view law enforcement involvement and criminal prosecution, and what impact do these views have on case processing?. Journal of Elder Abuse \& Neglect 2013;25(3):254-80.

\section{Jackson 2013a \{published data only\}}

Jackson SL, Hafemeister TL. Enhancing the safety of elderly victims after the close of an APS investigation. Journal of Interpersonal Violence 2013;28(6):1223-39.

\section{Jamieson 2004 \{published data only\}}

Jamieson W, Hart L. Preventing and Responding to Abuse in Long-Term Care Facilities: Lessons Learned From the Abuse Prevention in Long-Term Care (APLTC) Project. Ottawa, Ontario, Canada: National Clearinghouse on Family Violence, Family Violence Prevention Unit, Public Health Agency of Canada, 2004.

\section{Jenkens 2005 \{published data only\}}

Jenkens R, Carder PC, Maher L. The coming home program. Journal of Housing for the Elderly 2005;18:179-201. [DOI: 10.1300/J081v18n03_08]

\section{Jogerst 1997 \{published data only\}}

Jogerst GJ, Ely JW. Home visit program for teaching elder abuse evaluations. Family Medicine 1997;29(9):634-9.

\section{Jogerst 2001 \{published data only\}}

Jogerst G, Daly JM, Ingram J. National elder abuse questionnaire: Summary of adult protective service investigator responses. Journal of Elder Abuse \& Neglect 2001;13(4):59-71.

\section{Jogerst 2003 \{published data only\}}

Jogerst GJ, Daly JM, Brinig MF, Dawson JD, Schmuch GA, Ingram JG. Domestic elder abuse and the law. American Journal of Public Health 2003;93(12):2131-6.
Jogerst 2004 \{published data only\}

Jogerst GJ, Daly JM, Dawson JD, Brinig MF, Schmuch G, PeekeAsa C. APS investigative systems associated with county reported domestic elder abuse. Journal of Elder Abuse \& Neglect 2004;16(3):1-17.

\section{Jogerst 2008 \{published data only\}}

Jogerst GJ, Daly JM. County government resources associated with dependent-adult abuse investigations in lowa. Journal of Elder Abuse \& Neglect 2008;20(3):251-64.

\section{Jogerst 2008a \{published data only\}}

Jogerst GJ, Daly JM, Hartz AJ. State policies and nursing home characteristics associated with rates of resident mistreatment. Journal of the American Medical Directors Association 2008;9(9):648-56.

Johnson 1990 \{published data only\}

Johnson TF. Elder Mistreatment Identification Instruments. Journal of Elder Abuse \& Neglect 1990;1(4):15-36.

\section{Jones 1988 \{published data only\}}

Jones J, Dougherty J, Schelble D, Cunningham W. Emergency department protocol for the diagnosis and evaluation of geriatric abuse. Annals of Emergency Medicine 1988;17(10):1006-15.

\section{Joubert 2003 \{published data only\}}

Joubert J, Lindgren P. HEAL: an intervention project addressing elder abuse in South Africa. Intercom 2003;10(3):4-6.

\section{Joubert 2009 \{published data only\}}

Joubert L, Posenelli S. Responding to a "window of opportunity": the detection and management of aged abuse in an acute and subacute health care setting. Social Work in Health Care 2009;48(7):702-14.

\section{Kalavar 2012 \{published data only\}}

Kalavar JM, Jamuna D, Ejaz FK. Elder abuse in India: extrapolating from the experiences of seniors in India's "pay and stay" homes. Journal of Elder Abuse \& Neglect 2012;25(1):3-18.

\section{Karp 2006 \{published data only\}}

Karp N, Wood E. Guardianship Monitoring: A National Survey of Court Practices. Washington, DC: AARP Public Policy Institute, June 2006. [http://assets.aarp.org/rgcenter/consume/2006_14_ guardianship.pdf]

\section{Kassab 1999 \{published data only\}}

Kassab C, Vance L. An assessment of the effectiveness of an intergenerational program for youth. Psychological Reports 1999;84(1):198-200.

\section{Kasunic 2010 \{published data only\}}

Kasunic ML. Developing local elder abuse prevention coalitions. Victimization of the Elderly and Disabled 2010;13(3):1-46.

\section{Keller 1996 \{published data only\}}

Keller BH. Training course reduces abuse in nursing homes. Aging Magazine 1996;367:110-2. 
Keswani 1997 \{published data only\}

Keswani AN. Elder abuse crisis intervention techniques and their effectiveness. (M.S.G. Thesis). California: University of Southern California, United States, 1997.

\section{Kim 2010 \{published data only\}}

Kim KK. Development of a web-based education program for nurses working in nursing homes on human rights of older adults. Journal of Korean Academy of Nursing 2010;40(4):463-72.

\section{Kiosses 2010 \{published data only\}}

Kiosses DNP, Arean PAP, Teri L, Alexopoulos GSMD. Homedelivered problem adaptation therapy (PATH) for depressed, cognitively impaired, disabled elders: A preliminary study. American Journal of Geriatric Psychiatry 2010;18(11):988-98.

\section{Kono 2009 \{published data only\}}

Kono A, Fujita T, Tsumura C, Kondo T, Kushiyama K, Rubenstein LZ. Preventive home visit model targeted to specific care needs of ambulatory frail elders: preliminary report of a randomized trial design. Aging Clinical and Experimental Research 2009;21(2):167-73.

\section{Kue 2009 \{published data only\}}

Kue R, Ramstrom E, Weisberg S, Restuccia M. Evaluation of an emergency medical services based social services referral program for elderly patients. Prehospital Emergency Care 2009;13(3):273-9.

\section{Kurrle 1993 \{published data only\}}

Kurrle SE. Responding to elder abuse-A follow-up study of interventions and outcomes. Australasian Journal on Ageing 1993;12(4):5-9.

\section{Kurrle 1997 \{published data only\}}

Kurrle SE, Sadler PM, Lockwood K, Cameron ID. Elder abuse: Prevalence, intervention and outcomes in patients referred to four aged care assessment teams. Medical Journal of Australia 1997;166(3):119-22.

\section{Laditka 2002 \{published data only\}}

Laditka SB, Fischer M, Mathews KB, Sadlik JM, Warfel ME. There's no place like home: evaluating family medicine residents' training in home care. Home Health Care Services Quarterly 2002;21(2):1-17.

\section{Lantz 1997 \{published data only\}}

Lantz MS, Buchalter EN, McBee L. The Wellness Group: a novel intervention for coping with disruptive behavior among [corrected] elderly nursing home residents. Gerontologist 1997;37(4):551-6.

\section{Lithwick 2000 \{published data only\}}

Lithwick M, Beaulieu M, Gravel S, Straka SM. The mistreatment of older adults: Perpetrator-Victim relationships and interventions. Journal of Elder Abuse \& Neglect 2000;11(4):95-112. [DOI: 10.1300/J084v11n04_07]

\section{López 2008 \{published data only\}}

López J, Crespo M. Analysis of the efficacy of a psychotherapeutic program to improve the emotional status of caregivers of elderly dependent relatives. Aging \& Mental Health 2008;12(4):451-61.

Lori 2011 \{published data only\}

Lori AS, Pamela BT. Final Technical Report to the National Institute of Justice on: A Multi-Site Assessment of Five Court-Focused Elder Abuse Initiatives. American Bar Association and University of Kentucky Research Foundation, 2011. [http://www.americanbar.org/content/ dam/aba/uncategorized/2011/2011_aging_ea_multi_ assess.authcheckdam.pdf]

Malks 2002 \{published data only\}

Malks B, Schmidt CM, Austin MJ. Elder abuse prevention: a case study of the Santa Clara County Financial Abuse Specialist Team (FAST) Program. Journal of Gerontological Social Work 2002;39(3):23-40.

Malks 2010 \{published data only\} Malks BF, Strobel DM, Leung Y, Court MW, Morris JR, May G, et al. Changing systems to address elder abuse: examples from aging services, the courts, the long-term care Ombudsman, and the faith community. Journal of Elder Abuse \& Neglect 2010;22(3-4):306-27.

\section{Manthorpe 2013 \{published data only\}}

Manthorpe J, Samsi K, Rapaport J. "Capacity is key": investigating new legal provisions in England and Wales for adult safeguarding. Journal of Elder Abuse \& Neglect 2013;25(4):355-73.

\section{Mariam 2015 \{published data only\}}

Mariam LM, McClure R, Robinson JB, Yang JA. Eliciting change in at-risk elders (ECARE): evaluation of an elder abuse intervention program. Journal of Elder Abuse \& Neglect 2015;27(1):19-33.

\section{Martin-Carrasco 2009 \{published data only\}}

Martin-Carrasco M, Martin MF, Valero CP, Millan PR, Garcia CI, Montalban SR, et al. Effectiveness of a psychoeducational intervention program in the reduction of caregiver burden in Alzheimer's disease patients' caregivers. International Journal of Geriatric Psychiatry 2009;24(5):489-99.

Mason 2003 \{published data only\}

Mason A. S.E.A.M., Stop Elder Abuse and Mistreatment: a psycho-educational program for abusers of the elderly. Victimization of the Elderly and Disabled 2003;5(5):67.

\section{Mason 2007 \{published data only\}}

Mason A, Weatherly H, Spilsbury K, Golder S, Arksey H, Adamson J, et al. The effectiveness and cost-effectiveness of respite for caregivers of frail older people. Journal of the American Geriatric Society 2007;55(2):290-9.

McGarry 2007 \{published data only\}

McGarry J, Simpson C. Nursing students and elder abuse: developing a learning resource. Nursing Older People 2007;19:27-30. 


\section{McGarry 2009 \{published data only\}}

McGarry J, Simpson C. Raising awareness of elder abuse in the community practice setting. British Journal of Community Nursing 2009;14(7):305-8.

\section{Mills 2012 \{published data only\}}

Mills WL, Roush RE, Moye J, Kunik ME, Wilson NL, Taffet GE, et al. An educational program to assist clinicians in identifying elder investment fraud and financial exploitation. Gerontology and Geriatrics Education 2012;33(4):351-63.

\section{Morris 2010 \{published data only\}}

Morris JR. The Bet Tzedek legal services model: how a legal services model addresses elder abuse and neglect. Journal of Elder Abuse \& Neglect 2010;22(3-4):275-80.

\section{Murphy 2015 \{published data only\}}

Murphy KP, Burnett J, Xia R, Hunt J, Dyer CB. Teaching elder abuse to interprofessional healthcare providers-does an educational intervention make a difference?. Journal of the American Geriatrics Society 2015;63:S49.

\section{NAAPSA 2003 \{published data only\}}

National Association of Adult Protective Services Administrators (NAAPSA). Report on State Adult Protective Services Response to Financial Exploitation of Vulnerable Adults 2003. Washington, D.C: National Center on Elder Abuse (NCEA), July, 2003.

\section{Nakanishi 2009 \{published data only\}}

Nakanishi M, Hoshishiba Y, Iwama N, Okada T, Kato E, Takahashi H. Impact of the elder abuse prevention and caregiver support law on system development among municipal governments in Japan. Health Policy 2009;90(23):254-61.

\section{Nakanishi 2010 \{published data only\}}

Nakanishi M, Nakashima T, Honda T. Disparities in systems development for elder abuse prevention among municipalities in Japan: Implications for strategies to help municipalities develop community systems. Social Sciences and Medicine 2010;71(2):400-4.

\section{Nakanishi 2013 \{published data only\}}

Nakanishi M, Nakashima T, Sakata N, Tsuchiya N, Takizawa K. Community-based system, reports, and substantiated cases of elder abuse: disparities between municipalities and relating factors in Japan. Journal of Aging and Social Policy 2013;25(3):234-47.

\section{Nakanishi 2013a \{published data only\}}

Nakanishi M, Nakashima T, Yamaoka Y, Hada K, Tanaka H. Systems development and difficulties in implementing procedures for elder abuse prevention among private community general support centers in Japan. Journal of Elder Abuse \& Neglect 2013;26(1):31-43.

\section{National Center of Elder Abuse 2002 \{published data only\}} National Center of Elder Abuse. Sentinels: Reaching Hidden Victims. Project Final Report. Washington DC: NCEA, 2002:32.

\section{Navarro 2010 \{published data only\}}

Navarro AE, Wilber KH, Yonashiro J, Homeier DC. Do we really need another meeting? Lessons from the Los Angeles County Elder Abuse Forensic Center. Gerontologist 2010;50(5):702-11.

\section{Navarro 2013 \{published data only\}}

Navarro AE, Gassoumis ZD, Wilber KH. Holding abusers accountable: an elder abuse forensic center increases criminal prosecution of financial exploitation. Gerontologist 2013;53(2):303-12

\section{Neale 1996 \{published data only\}}

Neale AV, Hwalek MA, Goodrich CS, Quinn KM. The Illinois elder abuse system: Program description and administrative findings. Gerontologist 1996;36(4):502-11.

\section{Nelson 1992 \{published data only\}}

Nelson GM. Training adult-service social workers in the public sector: a core curriculum for effective geriatric social work practice. Educational Gerontology 1992;18(2):163-76.

\section{Nerenberg 1986 \{published data only\}}

Nerenberg L, Barry M. Community Outreach Booklet: Developing a Public Awareness Campaign: Elder Abuse Awareness. San Francisco, CA: Consortium for Elder Abuse Prevention, Coordinating Agency: Mount Zion Hospital and Medical Center, 1986.

\section{Nusbaum 2007 \{published data only\}}

Nusbaum NJ, Cheung VM, Cohen J, Keca M, Mailey B. Role of first responders in detecting and evaluating elders at risk. Archives of Gerontology and Geriatrics 2006;43(3):361-7. [DOI: 10.1016/j.archger.2006.01.001]

* Nusbaum NJ, Mistretta M, Wegner J. Educational intervention for police and firefighters for elders at risk: limits of education alone as a strategy for behavior change. Educational Gerontology 2007;33(10):801-9. [DOI: 10.1080/03601270701568798]

Payne 2007 \{published data only\}

Payne B, Carmody DC, Plichta S, Vandecar-Burdin T. Domestic violence training policies - Influence on participation in training and awareness of abuse. Affilia-Journal of Women and Social Work 2007;22(3):292-301.

Payne 2010 \{published data only\} Payne KL, Prentice-Dunn S, Allen RS. A comparison of two interventions to increase completion of advance directives. Clinical Gerontologist: The Journal of Aging and Mental Health 2010;33:49-61.

\section{Payne 2012 \{published data only\}}

Payne BK, Strasser SM. Financial exploitation of older persons in adult care settings: comparisons to physical abuse and the justice system's response. Journal of Elder Abuse \& Neglect 2012;24(3):231-50.

Pellfolk 2010 \{published data only\}

Pellfolk TJE, Gustafson Y, Bucht G, Karlsson S. Effects of a restraint minimization program on staff knowledge, attitudes, 
and practice: a cluster randomized trial. Journal of the American Geriatrics Society 2010;58(1):62-9.

\section{Perttu 1996 \{published data only\}}

Perttu S. Abuse of the elderly: services provided for victims in a Finnish nursing home--1992-1993. Journal of Elder Abuse \& Neglect 1996;8(2):23-31.

\section{Phillips 2008 \{published data only\}}

Phillips L R. Abuse of aging caregivers - Test of a nursing intervention. Advances in Nursing Science 2008;31:164-81.

Phillips 2011 \{published data only\}

Phillips LR, Guo GF. Mistreatment in assisted living facilities: complaints, substantiations, and risk factors. Gerontologist 2011;51(3):343-53.

\section{Pillemer 1993 \{published data only\}}

Pillemer K, Hudson B. A model abuse prevention program for nursing assistants. Gerontologist 1993;33(1):128-31.

\section{Pitkala 2011 \{published data only\}}

Pitkala $\mathrm{KH}$, Routasalo P, Kautiainen $\mathrm{H}$, Sintonen $\mathrm{H}$, Tilvis RS. Effects of socially stimulating group intervention on lonely, older people's cognition: a randomized, controlled trial. American Journal of Geriatric Psychiatry 2011 Jul;19(7):654-63. [DOI: 10.1097/JGP.0b013e3181f7d8b0]

Plotkin 1996 \{published data only\}

Plotkin MR. Improving the police response to domestic elder abuse victims. Aging 1996;367:28-32.

\section{Preston-Shoot 2002 \{published data only\}}

Preston-Shoot M, Wigley V. Closing the circle: social workers' responses to multi-agency procedures on older age abuse. British Journal of Social Work 2002;32(3):299.

Price 1997 \{published data only\}

Price G, Fox C. Massachusetts Bank Reporting Project: an edge against elder financial exploitation. Journal of Elder Abuse \& Neglect 1997;8(4):59-71.

\section{Queensland Government Department of Families 2001 \{published data only\}}

Queensland Government Department of Families. The Strategic Plan for the Prevention of Elder Abuse in Queensland. Brisbane: Queensland Government Department of Families, 2001. [http://pandora.nla.gov.au/pan/61190/20060731-0000/ www.communities.qld.gov.au/seniors/publications/ documents/pdf/strategic_plan_prev_elder_abuse.pdf]

\section{Rabinowitz 2006 \{published data only\}}

Rabinowitz YG, Mausbach BT, Coon DW, Depp C, Thompson LW, Gallagher-Thompson D. The moderating effect of self-efficacy on intervention response in women family caregivers of older adults with dementia. American Jornal of Geriatric Psychiatry 2006 Aug;14(8):642-9.

\section{Radcliff 2013 \{published data only\}}

Radcliff TA, White A, West DR, Hurd D, Cote MJ. Evaluation of a seven state criminal history screening pilot program for long-term care workers. Journal of Elder Abuse \& Neglect 2013;25(5):375-95.

Reap 2002 \{published data only\}

Reap AMC, Browne KD. The effectiveness of psychological interventions with individuals who physically abuse or neglect their elderly dependents. Journal of Interpersonal Violence 2002;17(4):416-31.

Reay 2002 \{published data only\}

* Reay AMC, Browne KD. The effectiveness of psychological interventions with individuals who physically abuse or neglect their elderly dependents. Journal of Interpersonal Violence 2002;17(4):416-31. [DOI: 10.1177/0886260502017004005]

\section{Riessman 1982 \{published data only\}}

Riessman F, Moody H, Worthy EH. Self-help and the Elderly: Considerations for Practice and Policy. Washington, DC: National Policy Center on Education, Leisure and Continuing Opportunities for Older Americans, National Council on the Aging, 1982.

\section{Roush 2012 \{published data only\}}

Roush RE, Moye JA, Mills WL, Kunik ME, Wilson NL, Taffet GE, et al. Why clinicians need to know about the elder investment fraud and financial exploitation program. Generations 2012;36(2):94-7.

\section{Saltz 2007 \{published data only\}}

Saltz CC, Davis P, Jackson L, Bach MS. Spirit of aging rising: cross-cutting thematic modules to enrich foundation graduate social work courses. Journal of Gerontological Social Work 2007;48(3-4):299-309.

\section{Sanders 2008 \{published data only\}}

Sanders S, Dorfman LT, Ingram JG. An evaluation of the GeroRich program for infusing social work curriculum with aging content. Gerontology and Geriatrics Education 2008;28:22-38

\section{Schaffer 1999 \{published data only\}}

Schaffer J. Older and isolated women and domestic violence project. Journal of Elder Abuse \& Neglect 1999;11(1):59-77.

\section{Schonfeld 2006 \{published data only\}}

Schonfeld L, Larsen RG, Stiles PG. Behavioral health services utilization among older adults identified within a state abuse hotline database. Gerontologist 2006;46(2):193-9.

\section{Scogin 1990 \{published data only\}}

Scogin F, Beall C, Bynum J, Stephens G, Grote NP, Baumhover LA, et al. Training for abusive caregivers. Journal of Elder Abuse \& Neglect 1990;1(4):73-86.

\section{Scogin 1990a \{published data only\}}

Scogin F, Stephens G, Bynum J. Training caregivers: The development of an elder abuse prevention program. Journal of Health and Human Resources Administration 1990;12(4):499-508. 
Seamon 1996 \{published data only\}

Seamon JP. The detection and reporting of elder abuse and neglect: A training video for prehospital personnel [1377941]. United States -- Michigan:Grand Valley State University (MHS thesis) 1996:28p.

\section{Seamon 1997 \{published data only\}}

Seamon JP, Jones JS, Chun E, Krohmer JR. Identifying victims of elder abuse and neglect: a training video for prehospital personnel. Prehospital \& Disaster Medicine 1997;12(4):269-73.

\section{Shah 2008 \{published data only\}}

Shah MN, Rajasekaran K, Sheahan WD, III, Wimbush T, Karuza J. The effect of the geriatrics education for emergency medical services training program in a rural community. Journal of the American Geriatrics Society 2008;56(6):1134-9.

\section{Shefet 2007 \{published data only\}}

Shefet D, Dascal-Weichhendler H, Rubin O, Pessach N, Itzik D, Benita S, et al. Domestic violence: a national simulation-based educational program to improve physicians' knowledge, skills and detection rates. Medical Teacher 2007;29(5):E133-E8.

Shugarman 2003 \{published data only\}

Shugarman LR, Fries BE, Wolf RS, Morris JN. Identifying older people at risk of abuse during routine screening practices. Journal of the American Geriatrics Society 2003;51(1):24-31.

\section{Signe 2008 \{published data only\}}

Signe A, Elmstahl S. Psychosocial intervention for family caregivers of people with dementia reduces caregiver's burden: development and effect after 6 and 12 months. Scandinavian Journal of Caring Sciences 2008;22:98-109.

\section{Simon 1992 \{published data only\}}

Simon ML. Exploratory Study of Adult Protective Services Programs' Repeat Elder Abuse Clients. Washington DC: American Association of Retired Persons, Public Policy Institute, 1992.

\section{Smith 2010 \{published data only\}}

Smith MK, Davis BH, Blowers A, Shenk D, Jackson K, Kalaw K. Twelve important minutes: introducing enhanced online materials about elder abuse to nursing assistants. Journal of Continuing Education in Nursing 2010;41(6):281-8.

\section{Spijker 2011 \{published data only\}}

Spijker A, Wollersheim H, Teerenstra S, Graff M, Adang E, Verhey $F$, et al. Systematic care for caregivers of patients with dementia: a multicenter, cluster-randomized, controlled trial. American Journal of Geriatric Psychiatry 2011;19(6):521-31.

\section{Spijker 2013 \{published data only\}}

Spijker A, Teerenstra S, Wollersheim H, Adang E, Verhey F, Vernooij-Dassen M. Influence of adherence to a systematic care program for caregivers of dementia patients. American Journal of Geriatric Psychiatry 2013;21(1):26-36. [DOI: 10.1016/ j.jagp.2012.10.003]

\section{Stark 2012 \{published data only\}}

Stark S. Elder abuse: Screening, intervention and prevention. Nursing 2012;42(10):24-29.

\section{Strümpel 2011 \{published data only\}}

Strümpel C, Hackl C. The Breaking the Taboo projects - raising awareness of, and training staff in, community health and care services on violence against older women within families. Journal of Adult Protection 2011;13(6):323-35.

Sugita 2011 \{published data only\}

Sugita JA, Garrett MD. Elder abuse and oral health care providers: an intervention to increase knowledge and selfperceived likelihood to report. Journal of Elder Abuse \& Neglect 2011;24(1):50-64.

Tapper 2010 \{published data only\}

Tapper L. Using family group conferences in safeguarding adults. Journal of Adult Protection 2010;12(1):27-31.

\section{Teaster 2003 \{published data only\}}

Teaster PB, Nerenberg L. A national look at elder abuse multidisciplinary teams. Report for the National Committee for the Prevention of Elder Abuse Partner National Center on Elder Abuse. Washington, D.C: National Center on Elder Abuse (NCEA), 2003.

\section{Teaster 2003a \{published data only\}}

Teaster PB. A Response to the Abuse of Vulnerable Adults: The 2000 Survey of State Adult Protective Services. Washington DC: National Center on Elder Abuse (NCEA), 2003.

Teaster 2004 \{published data only\}

Teaster PB, Roberto KA. Sexual abuse of older adults: APS cases and outcomes. Gerontologist 2004;44(6):788-96.

Teaster 2004a \{published data only\}

Teaster PB, Roberto KA. Sexual abuse of older women living in nursing homes. Journal of Gerontological Social Work 2004;40(4):105-19.

Teaster 2006 \{published data only\}

Teaster PB, Dugar T, Mendiondo M, Abner E, Cecil K, Otto J. Abuse of Adults Age 60+: The 2004 Survey of Adult Protective Services - Abuse of Adults 60 Years of Age and Older. National Center on Elder Abuse (NCEA), February 2006.

Teaster 2010 \{published data only\}

Teaster PB, Wangmo T. Kentucky's Local Elder Abuse Coordinating Councils: a model for other states. Journal of Elder Abuse \& Neglect 2010;22(1-2):191-206.

Teitelman 2000 \{published data only\}

Teitelman JL, O'Neill P. Elder and adult sexual abuse: a model curriculum for adult services/adult protective services workers. Journal of Elder Abuse \& Neglect 2000;11(3):91-100.

Testad 2005 \{published data only\}

Testad I, Aasland AM, Aarsland D. The effect of staff training on the use of restraint in dementia: a single-blind randomised 
controlled trial. International Journal of Geriatric Psychiatry 2005;20(6):587-90.

\section{Tompkins 2009 \{published data only\}}

Tompkins SA, Bell PA. Examination of a psychoeducational intervention and a respite grant in relieving psychosocial stressors associated with being an Alzheimer's caregiver. Journal of Gerontological Social Work 2009;52(2):89-104.

\section{Uva 1996 \{published data only\}}

Uva JL, Guttman T. Elder abuse education in an emergency medicine residency program. Academic Emergency Medicine 1996 Aug;3(8):817-9.

\section{Vaccaro 1990 \{published data only\}}

Vaccaro FJ. Application of social skills training in a group of institutionalized aggressive elderly subjects. Psychology and Aging 1990;5(3):369-78.

\section{Vaccaro 1992 \{published data only\}}

Vaccaro FJ. Physically aggressive elderly: a social skills training program. Journal of Behavior Therapy and Experimental Psychiatry 1992;23(4):277-88.

\section{Victoria Department for Victorian Communities 2006 \{published data only\}}

Victoria Department for Victorian Communities. Supporting the Safety and Dignity of Senior Victorians: Victorian Government Response to the Report of the Elder Abuse Prevention Project. Melbourne, Victoria: Office of Senior Victorians, 2006. [http:// www.seniorsonline.vic.gov.au/]

Vinton 1993 \{published data only\}

Vinton L. Educating case managers about elder abuse and neglect. Journal of Case Management 1993;2(3):101-5.

\section{Vinton 1997 \{published data only\}}

Vinton L, Altholz JAS, Lobell-Boesch T. Five-year follow up study of domestic violence programming for older battered women. Journal of Women and Aging 1997;9(1-2):3-15.

\section{Vladescu 2000 \{published data only\}}

Vladescu D, Eveleigh K, Ploeg J, Patterson C. An evaluation of a client-centered case management program for elder abuse. Journal of Elder Abuse \& Neglect 2000;11(4):5-22.

\section{Wagenaar 2009 \{published data only\}}

Wagenaar DB, Rosenbaum R, Page C, Herman S. Elder abuse education in residency programs: how well are we doing?. Academic Medicine 2009;84:611-8.

\section{Wasylkewycz 1994 \{published data only\}}

Wasylkewycz MN. The elder abuse resource centre, a coordinated community response to elder abuse. Journal of Elder Abuse \& Neglect 1994;5(4):21-33.

\section{Watson 1994 \{published data only\}}

Watson MM, Cesario TC, Ziemba S, McGovern P. Elder abuse in long-term care environments. Journal of Elder Abuse \& Neglect 1994;5(4):95-111.
Weiner 1991 \{published data only\}

Weiner A. A community-based education model for identification and prevention of elder abuse. Journal of Gerontological Social Work 1991;16(3-4):107-19.

Wiglesworth 2006 \{published data only\}

Wiglesworth A, Mosqueda L, Burnight K, Younglove T, Jeske D. Findings from an elder abuse forensic center. Gerontologist 2006;46(2):277-83.

Williams 2004 \{published data only\}

Williams K, Kemper S, Hummert ML. Enhancing communication with older adults: overcoming elderspeak. Journal of Gerontological Nursing 2004;30(10):17-25.

Williams 2007 \{published data only\} Williams B, Anderson MC, Day R. Undergraduate nursing students' knowledge of and attitudes toward aging: comparison of context-based learning and a traditional program. Journal of Nursing Education 2007;46(3):115-20.

Wolf 2000 \{published data only\} Wolf RS, Pillemer K. Elder abuse and case outcome. Journal of Applied Gerontology 2000;19(2):203-20.

Yan 2014 \{published data only\}

Yan E. Abuse of older persons with dementia by family caregivers: results of a 6-month prospective study in Hong Kong. International Journal of Geriatric Psychiatry 2014;29(10):1018-27.

\section{References to ongoing studies}

Loh 2015 \{published data only\}

Loh DA, Choo WY, Hairi NN, Othman S, Mohd Hairi F, Mohd Mydin $\mathrm{FH}$, et al. A cluster randomized trial on improving nurses' detection and management of elder abuse and neglect (I-NEED): study protocol. Journal of Advanced Nursing 2015;71(11):2661-72.

\section{Additional references}

\section{Acierno 2010}

Acierno R, Hernandez MA, Amstadter AB, Resnick HS, Steve K, Muzzy W, et al. Prevalence and correlates of emotional, physical, sexual, and financial abuse and potential neglect in the United States: the National Elder Mistreatment Study. American Journal of Public Health 2010;100:292-7.

\section{Action on Elder Abuse 1995a}

Action on Elder Abuse. What is elder abuse?. What is Elder Abuse? Action on Elder Abuse Bulletin. Vol. 11 (May-June), UK: Action on Elder Abuse, 1995.

\section{Alt 2011}

Alt KL, Nguyen AL, Meurer LN. The effectiveness of educational programs to improve recognition and reporting of elder abuse and neglect: A systematic review of the literature. Journal of Elder Abuse \& Neglect 2011;23:213-33. 


\section{American Bar Association 2006}

American Bar Association Commission on Law and Aging. Analysis of state adult protective services laws. Available from http://www.elderabusecenter.org/default.cfm? p 1/4statelawsanalysis.cfm 2006 (accessed on 30 November 2011).

\section{Ansello 2010}

Ansello EF, O'Neill P. Abuse, neglect, and exploitation: considerations in aging with lifelong disabilities. Journal of Elder Abuse \& Neglect 2010;22:105-30. [DOI: $10.1080 / 08946560903436395]$

\section{Armstrong 2011}

Armstrong R, Waters E, Doyle J. Chapter 21: Reviews in health promotion and public health. In: Higgins JPT, Green S editor(s) Cochrane Handbook for Systematic Reviews of Interventions. Chichester (UK): John Wiley \& Sons, 2011.

\section{Baker 1975}

Baker AA. Granny bashing. Modern Geriatrics 1975;5:20-4.

\section{Baker 2015}

Baker PRA, Francis DP, Soares J, Weightman AL, Foster C. Community wide interventions for increasing physical activity. Cochrane Database of Systematic Reviews 2015, Issue 1. [DOI: 10.1002/14651858.CD008366.pub3]

\section{BBA 2010}

British Banking Association (BBA). Safeguarding vulnerable customers. Available from www.bba.org.uk/download/1208 2010 (accessed 4/12/2012).

\section{Biggs 2009}

Biggs S, Manthorpe J, Tinker A, Doyle M, Erens B. Mistreatment of older people in the United Kingdom: findings from the first National Prevalence Study. Journal of Elder Abuse \& Neglect 2009;21(1):1-14

\section{Bonnie 2003}

Bonnie RJ, Wallace RB. Elder Mistreatment: Abuse, Neglect, and Exploitation in an Aging America. Washington, DC: National Academies Press, 2003.

\section{Browne 1997}

Browne K, Herbert M. Preventing Family Violence. Chichester (UK): John Wiley \& Sons Ltd., 1997.

\section{Burston 1975}

Burston GR. "Granny bashing”. British Medical Journal 1975;3:352.

\section{Butchart 2008}

Butchart A, Brown D, Khanh-Huynh A, Corso P, Floquin N, Muggah R. Manual for Estimating the Economic Costs of Injuries Due to Interpersonal and Self-directed Violence. Geneva: World Health Organization, 2008.

\section{Cooper 2008}

Cooper C, Selwood A, Livingston G. The prevalence of elder abuse and neglect: a systematic review. Age and Ageing 2008;37:151-60.

\section{Cooper 2009}

Cooper C, Selwood A, Blanchard M, Walker Z, Blizard R, Livingston $\mathrm{G}$. Abuse of people with dementia by family carers: representative cross sectional survey. BMJ 2009;338:b155. [DOI: 10.1136/bmj.b155]

\section{CPHG 2011}

Cochrane Public Health Group. Guide for developing a Cochrane review protocol. Available at http://ph.cochrane.org/ sites/ph.cochrane.org/files/uploads/Guide\%20for\%20PH \%20protocol_Nov\%202011_final\%20for\%20website.pdf 2011 (accessed on 16/07/2012).

\section{Curran 2007}

Curran JA, Dartnell J, Magee K, Sinclair D, McGrath PJ. Organisational and professional interventions to promote the uptake of evidence in emergency care: Effects on professional practice and health outcomes. Cochrane Database of Systematic Reviews 2007, Issue 2. [DOI: 10.1002/14651858.CD006557]

\section{Dakin 2009}

Dakin E, Pearlmutter S. Older women's perceptions of elder maltreatment and ethical dilemmas in adult protective services: a cross-cultural, exploratory study. Journal of Elder Abuse \& Neglect 2009;21:15-57. [DOI: 10.1080/08946560802571896]

\section{Daly 2011}

Daly JM, Merchant ML, Jogerst GJ. Elder abuse research: A systematic review. Journal of Elder Abuse \& Neglect 2011;23:348-65.

\section{Desmarais 2007}

Desmarais SL, Reeves KA. Gray, black, and blue: the state of research and intervention for intimate partner abuse among elders. Behavioral Sciences \& the Law 2007;25:377-91.

\section{Doe 2009}

Doe SS, Han HK, McCaslin R. Cultural and ethical issues in Korea's recent elder abuse reporting system. Journal of Elder Abuse \& Neglect 2009;21:170-85. [DOI: 10.1080/08946560902780009]

\section{Dong 2007}

Dong X, Simon MA, Gorbien M, Percak J, Golden R. Loneliness in older Chinese adults: a risk factor for elder mistreatment. Journal of Women and Aging 2007;55:1831-5.

\section{Dong 2009}

Dong X, Beck T, Simon MA. Loneliness and mistreatment of older Chinese women: does social support matter?. Journal of Women and Aging 2009;21:293-302.

\section{Dong 2012}

Dong X, Simon MA, Evans D. Prospective study of the elder self-neglect and ED use in a community population. American Journal of Emergency Medicine 2012;30:553-61. 
Dong 2013

Dong XinQi, Simon MA. Association between elder abuse and use of ED: findings from the Chicago Health and Aging Project. American Journal of Emergency Medicine 2013;31:693-8.

\section{Dugan 2003}

Dugan L, Nagin DS, Rosenfeld R. Do domestic violence services save lives?. National Institute of Justice Journal 2003;250:20-5.

\section{Dyer 2000}

Dyer CB, Pavlik VN, Murphy KP, Hyman DJ. The high prevalence of depression and dementia in elder abuse or neglect. Journal of the American Geriatrics Society 2000;48:205-8.

\section{Dyer 2005}

Dyer CB, Heisler CJ, Hill CA, Kim LC. Community approaches to elder abuse. Clinics in Geriatrics Medicine 2005;21:429-47.

\section{Dyer 2007}

Dyer CB, Pickens S, Burnett J. Vulnerable elders. JAMA 2007;298:1448-50.

\section{Egger 1998}

Egger M, Smith GD. Meta-analysis bias in location and selection of studies. BMJ 1998;316:61-6.

\section{EPOC 2011}

EPOC. Worksheets for preparing a summary of findings using GRADE. Available from http://www.ph.cochrane.org/ sites/ph.cochrane.org/files/uploads/Guide\%20for\%20PH \%20protocol_booklet_UpdatedJan52011.pdf 2011 (accessed 19 September 2011).

\section{EPOC 2015}

Effective Practice, Organisation of Care (EPOC). EPOC Resources for review authors. Available at: http://epoc.cochrane.org/epocspecific-resources-review-authors 2015.

\section{Fulmer 2008a}

Fulmer T. Barriers to neglect and self-neglect research. Journal of the American Geriatrics Society 2008;56 Suppl 2:S241-3.

\section{Gordon 2001}

Gordon RM, Brill D. The abuse and neglect of the elderly. International Journal of Law and Psychiatry 2001;24:183-97.

\section{Hansberry 2005}

Hansberry MR, Chen E, Gorbien MJ. Dementia and elder abuse. Clinics in Geriatric Medicine 2005;21:315-32.

\section{Heathcote 2011}

Heathcote E. Old age and the city. BMJ 2011;343:d4418.

\section{Higgins 2008}

Higgins JPT, Green S (editors). Cochrane Handbook for Systematic Reviews of Interventions. Chichester (UK): John Wiley \& Sons, 2008.

\section{HSE 2009}

HSE Elder Abuse Service (HSE). Open your eyes. Health Service Executive. Available from http://www.hse.ie/eng/services/
Publications/services/Older/openyoureyes 2009 (accessed 28 December 2011).

\section{Jackson 2009}

Jackson L. The Cost of Elder Abuse in Queensland: Who Pays and How Much?. Brisbane: Elder Abuse Prevention Unit, 2009.

\section{Jackson 2011}

Jackson SL, Hafemeister TL. Risk factors associated with elder abuse: the importance of differentiating by type of elder maltreatment. Violence and Victims 2011;26:738-57.

\section{Jeon 2005}

Jeon $\mathrm{YH}$, Brodaty $\mathrm{H}$, Chesterson J. Respite care for caregivers and people with severe mental illness: literature review. Journal of Advanced Nursing 2005;49:297-306.

\section{Killick 2009}

Killick C, Taylor BJ. Professional decision making on elder abuse: systematic narrative review. Journal of Elder Abuse \& Neglect 2009;21:211-38.

\section{Koenig 2005}

Koenig RJ 3rd, DeGuerre CR. The legal and governmental response to domestic elder abuse. Clinics in Geriatrics Medicine 2005;21:383-98.

\section{Kosberg 2003}

Kosberg JI, Lowenstein A, Garcia JL, Biggs S. Study of elder abuse within diverse cultures. Journal of Elder Abuse \& Neglect 2003;15:71-89. [DOI: 10.1300/J084v15n03_05]

\section{Kurrle 2008}

Kurrle S, Naughtin G. An overview of elder abuse and neglect in Australia. Journal of Elder Abuse \& Neglect 2008;20:108-25. [DOI: 10.1080/08946560801974521]

\section{Lachs 2004}

Lachs MS, Pillemer K. Elder abuse. Lancet 2004;364:1263-72.

\section{Laumann 2008}

Laumann EO, Leitsch SA, Waite LJ. Elder mistreatment in the United States: prevalence estimates from a nationally representative study. Journals of Gerontology, Series B: Psychological Sciences and Social Sciences 2008;63:S248-54.

\section{Lowenstein 2009}

Lowenstein A, Eisikovits Z, Band-Winterstein T, Enosh G. Is elder abuse and neglect a social phenomenon? Data from the First National Prevalence Survey in Israel. Journal of Elder Abuse \& Neglect 2009;21:253-77.

\section{Luo 2011}

Luo Y, Waite LJ. Mistreatment and psychological well-being among older adults: exploring the role of psychosocial resources and deficits. Journals of Gerontology, Series B: Psychological Sciences and Social Sciences 2011;66(2):217-29.

\section{Moon 1993}

Moon A, Williams O. Perceptions of elder abuse and helpseeking patterns among African-American, Caucasian 
American, and Korean-American elderly women. Gerontologist 1993;33(3):386-95.

\section{Mouton 2004}

Mouton CP, Rodabough RJ, Rovi SL, Hunt JL, Talamantes MA, Brzyski RG, et al. Prevalence and 3-year incidence of abuse among postmenopausal women. American Journal of Public Health 2004;94:605-12.

\section{NCBDC 2012}

NCB Development Corporation (NCBDC). Coming Home Programme. Available from http://www.ncbdc.org/ default.aspx?id=64 2012 (accessed on 15 March 2012).

\section{NCEA 2006}

National Center on Elder Abuse. The 2004 Survey of State Adult Protective Services: Abuse of Adults 60 Years of Age and Older. Washington, DC: American Public Human Services Association, 2006.

\section{NZBA 2007}

New Zealand Bankers Association (NZBA). Voluntary guidelines to assist banks to meet the needs of older and disabled customers. Available from http://www.nzba.org.nz/bankingstandards/code-of-banking-practice/voluntary-guidelinesto-assist-banks-to-meet-the-needs-of-older-and-disabledcustomers/ 2007 (accessed on 16/06/2012).

\section{Penhale 2008}

Penhale B. Elder abuse in the United Kingdom. Journal of Elder Abuse \& Neglect 2008;20:151-68. [DOI: 10.1080/08946560801974653]

\section{Ploeg 2009}

Ploeg J, Fear J, Hutchison B, MacMillan H, Bolan G. A systematic review of interventions for elder abuse. Journal of Elder Abuse \& Neglect 2009;21:187-210. [DOI: 10.1080/08946560902997181]

\section{Podnieks 2008}

Podnieks E. Elder abuse: the Canadian experience. Journal of Elder Abuse \& Neglect 2008;20:126-50. [DOI: 10.1080/08946560801974612]

\section{Podnieks 2010}

Podnieks E, Penhale B, Goergen T, Biggs S, Han D. Elder mistreatment: an international narrative. Journal of Elder Abuse \& Neglect 2010;22:131-63. [DOI: 10.1080/08946560903436403]

\section{Porta 2014}

Porta Miquel. A Dictionary of Epidemiology. Oxford University Press, 2014.

\section{RevMan 2011 [Computer program]}

The Nordic Cochrane Centre, The Cochrane Collaboration. Review Manager (RevMan). Version 5.1. Copenhagen: The Nordic Cochrane Centre, The Cochrane Collaboration, 2011.

\section{Schiamberg 2000}

Schiamberg LB, Gans D. Elder abuse by adult children: An applied ecological framework for understanding contextual risk factors and the intergenerational character of quality of life. International Journal of Aging and Human Development 2000;50:329-59.

\section{Schiamberg 2012}

Schiamberg LB, Oehmke J, Zhang Z, Barboza GE, Griffore RJ, Von Heydrich L, et al. Physical abuse of older adults in nursing homes: a random sample survey of adults with an elderly family member in a nursing home. Journal of Elder Abuse \& Neglect 2012;24:65-83.

\section{Schneider 2010}

Schneider DC, Mosqueda L, Falk E, Huba GJ. Elder abuse forensic centers. Journal of Elder Abuse \& Neglect 2010;22:255-74.

\section{Schünemann 2011}

Schünemann HJ, Oxman AD, Vist GE, Higgins JPT, Deeks JJ, Glasziou P, et al. Chapter 12: Interpreting results and drawing conclusions. In: Higgins JPT, Green S (editors).. Cochrane Handbook for Systematic Reviews of Interventions. Version 5.1 [updated March 2011]. The Cochrane Collaboration. John Wiley \& Sons Ltd., 2011.

\section{Schünemann 2011a}

Schünemann H, Hill S, Guyatt G, Akl EA, Ahmed F. The GRADE approach and Bradford Hill's criteria for causation. Journal of Epidemiology and Community Health 2011;65:392-5.

\section{Soares 2010}

Soares JJF, Barros H, Torres-Gonzales F, Ioannidi-Kapolou E, Lamura G, Lindert J, et al. Abuse and Health in Europe. Kaunas: Lithuanian University of Health Sciences Press, 2010.

\section{Sterne 2011}

Sterne JAC, Egger M, Moher D. Chapter 10: Addressing reporting biases. In Higgins JPT, Green S (editors). Cochrane Handbook for Systematic Reviews of Interventions. Version 5.1 [updated March 2011]. The Cochrane Collaboration, 2011. Available from www.cochrane-handbook.org.

\section{Tugwell 2006}

Tugwell P, De Savigny D, Hawker G, Robinson V. Applying clinical epidemiological methods to health equity: the equity effectiveness loop. BMJ 2006;332:358-61.

\section{Twomey 2010}

Twomey MS, Jackson G, Li H, Marino T, Melchior LA, Randolph JF, et al. The successes and challenges of seven multidisciplinary teams. Journal of Elder Abuse \& Neglect 2010;22:291-305

\section{Ueffing 2011}

Ueffing E, Tugwell P, Welch V, Petticrew M, Kristjansson E for the Campbell and Cochrane Equity Methods Group. Equity Checklist for Systematic Review Authors. Version 2011-09-23. Available from http://www.equity.cochrane.org/ 2011 (accessed on $25 / 4 / 2012$ ). 


\section{UK Home Office 2012}

UK Home Office. Vetting and barring scheme. 2012. Available from http://www.homeoffice.gov.uk/crime/vetting-barringscheme/ (accessed on 14 March 2012).

\section{United Nations 2012}

United Nations. Department of Economic and Social Affairs. Data on older persons, 2012. Available from http:// social.un.org/index/Ageing/DataonOlderPersons.aspx (accessed on 30 April 2012).

\section{WHO 2002a}

World Health Organization (WHO). The Toronto declaration on the global prevention of elder abuse. Geneva: WHO, 2002.

\section{WHO 2002b}

World Health Organization/International Network for the Prevention of Elder Abuse (WHO/INPEA). Missing voices. Geneva: WHO, 2002.

\section{WHO 2011}

World Health Organization (WHO). European report on preventing elder maltreatment. Copenhagen: WHO Regional Office for Europe, 2011:87.

\section{Wilson 2003}

Wilson J, Micucci S. Interventions to prevent the recurrence of elder abuse. http://old.hamilton.ca/phcs/ephpp/

\section{CHARACTERISTICS OF STUDIES}

Characteristics of included studies [ordered by study ID]
ReviewsPortal.asp. Hamilton: City of Hamilton, Public Health and Community Services Department, 2003.

\section{Wolf 1999}

Wolf RS, Li D. Factors affecting the rate of elder abuse reporting to a State Protective Services Program. Gerontologist 1999;39:222-8.

\section{Wolf 2003}

Wolf R. In: Bonnie RJ, Wallace RB editor(s). Elder Abuse and Neglect: History and Concepts. Washington, DC: National Academies Press, 2003.

\section{Wu 2012}

Wu L, Chen H, Hu Y, Xiang H, Yu X, Zhang T, et al. Prevalence and associated factors of elder mistreatment in a rural community in People's Republic of China: a cross-sectional study. PLoS One 2012;7(3):e33857.

\section{Yan 2003}

Yan E, Tang CS. Proclivity to elder abuse: a community study on Hong Kong Chinese. Journal of Interpersonal Violence 2003;18:999-1017.

* Indicates the major publication for the study

\title{
Bartels 2005
}

\section{Methods}

\author{
Study Design \\ Controlled before-and-after (CBA) study \\ Study Location \\ New England, USA \\ Country
}

High income

Study Period

May 1998-2000

\section{Sampling Methods}

Thirteen Community mental health organisations $(\mathrm{CMHO})$ and home health care organisations $(\mathrm{HH}-$ CO) from three New England states were recruited. They were assigned to intervention and comparison groups matched on number of providers, type and service characteristics. Four CMHOs and three HHCOs were assigned to intervention condition, in which the primary clinicians conducted initial evaluations, quarterly outcome assessments, and treatment planning activities using the quality improvement kit. Four CMHOs and two HHCOs were assigned to usual care.

\section{Data collection method}


Data used to determine whether utilisation of the toolkit improved the quality of clinician practice were collected through (1) clinician interviews and (2) medical chart reviews. Consumer completed a 10-item self-report instrument.

\section{Inclusion criteria}

i.Elderly persons aged 60 or over with an Axis I diagnosis of a major psychiatric disorder, including Alzheimer and related dementia.

ii.Received mental health or home health services between May 1998 to 2000

iii. Eligible $\mathrm{CMHO}$ consumers included those who had an assigned clinician and received at least 3 hours of services per quarter; HHCO consumers received at least two visits per month

iv. Clinicians were eligible to participate in the study if they had two or more older adult consumers on their caseload and had received training in the procedures for the comparison or intervention condition.

\section{Ethics and informed consent}

Written consent from elderly persons obtained.

Participants

Settings

$\mathrm{CMHO}$ and $\mathrm{HHCO}$

\section{Sample size}

13 agencies, 44 clinicians, 100 elderly persons ( $>60$ of age)

\section{Participants' characteristics}

The participating clinicians were mostly female (84\%), 38.6\% social workers, $34.1 \%$ nurses, $11.4 \%$ with an advanced psychology degree, and $15.9 \%$ with other training. Clinicians involved had provided mental health services for almost 10.7 years $(S D=7.2)$ with the mean $(M)$ length of employment of 6.3 years $(S D=5.5)$. In the comparison group, clinicians had a heavier total caseload ( $M=38.4$ elderly people, $S D$ $=26.1)$, but the average older adult caseload was similar at $9.6(S D=10.2)$ older people per clinician.

The participating elderly people in the intervention group were older $(M=72.5$ years, $S D=8.8)$ than those in the comparison group $(M=68.7, S D=6.7)$, female $(85.2 \%$ versus $66.7 \%)$. There were no significant differences with respect to ethnicity, marital status, or diagnosis (psychotic disorders relative to non-psychotic disorders). Most participants were Caucasian (97\%) and 23\% were married, 31\% were widowed, $28 \%$ were divorced or separated, and $14 \%$ were never married.

Interventions

\section{Name of intervention}

None stated

\section{Type of intervention}

Program to increase detection of elder abuse

\section{Start date of Intervention}

May 1998 to 2000

\section{Duration}

24 months

\section{Aim of Intervention}

To improve mental health screening and service planning practices for older adults

\section{Description of cost and resources}


Bartels 2005 (Continued)

Not stated

Evidence of consideration to equity issues

None

Information of intervention intensity

Not stated

Assessment of intensity

Medium

\section{Component of intervention}

This intervention involves clinical practice improvement change on clinical assessment, service planning and outcome measures for older people. Twenty-two assessments and treatment planning domains were included within the assessment and planning tool kit.

\section{Outcomes}

\section{Outcomes}

\section{Primary}

Detection of abuse

\section{Secondary}

Not applicable

\section{Measures}

The clinicians used a Quality Improvement Tool Kit - developed by a multi-disciplinary team of clinicians, consumers, administrators, and clinical outcomes researchers

The elderly person completed a 10-item self-report instrument (Senior Outcomes Checklist) which contains general physical and emotional health items and satisfaction items which address perceived quality of care and benefit from services.

\section{Time points}

Two time points over 12-month period

\section{Baseline}

T1 duration 12 months (May 1998-1999)

\section{Follow-up}

T2 duration 12 months (May 1999 - 2000) $75 \%$ ). There was significant improvement in routine assessment of neglect and abuse for older adults (OR 6.5).

No adjustment was performed for baseline differences between the groups.

This study assessed a small sample of clinicians, imbalance in the number of elderly persons, clinicians, and agencies (CMHOs and HHCOs) in intervention and comparison group.

\section{Risk of bias}

Bias Authors' judgement Support for judgement


Bartels 2005 (Continued)

\begin{tabular}{lll}
$\begin{array}{l}\text { Random sequence genera- } \\
\text { tion (selection bias) }\end{array}$ & High risk & Was not performed. \\
\hline $\begin{array}{l}\text { Allocation concealment } \\
\text { (selection bias) }\end{array}$ & High risk & Was not performed. \\
\hline $\begin{array}{l}\text { Blinding of participants } \\
\text { and personnel (perfor- } \\
\text { mance bias) }\end{array}$ & High risk & Was not performed. \\
All outcomes & & \\
\hline
\end{tabular}

\begin{tabular}{lll}
\hline $\begin{array}{l}\text { Blinding of outcome as- } \\
\text { sessment (detection bias) } \\
\text { All outcomes }\end{array}$ & High risk & Was not performed. \\
\hline $\begin{array}{l}\text { Incomplete outcome data } \\
\text { (attrition bias) }\end{array}$ & Low risk & No attrition in the study \\
All outcomes & &
\end{tabular}

\begin{tabular}{lll}
\hline $\begin{array}{l}\text { Selective reporting (re- } \\
\text { porting bias) }\end{array}$ & Low risk & All intended outcomes were reported. \\
\hline $\begin{array}{l}\text { Baseline outcome mea- } \\
\text { surements similar (selec- } \\
\text { tion bias) }\end{array}$ & High risk & $\begin{array}{l}\text { There were significant differences between the intervention and control } \\
\text { groups at baseline. }\end{array}$ \\
\hline $\begin{array}{l}\text { Baseline characteristic } \\
\text { similar (selection bias) }\end{array}$ & High risk & $\begin{array}{l}\text { The intervention group had large, medium and small amount of caseloads, } \\
\text { whereas the control group only had small and large ones. There are differ- } \\
\text { ences in the number of clinicians and average elderly persons per clinicians } \\
\text { between the intervention and control groups. }\end{array}$ \\
\hline
\end{tabular}

\begin{tabular}{|c|c|c|}
\hline $\begin{array}{l}\text { Knowledge of allocated in- } \\
\text { tervention adequately pre- } \\
\text { vented during the study } \\
\text { (detection Bias) }\end{array}$ & High risk & $\begin{array}{l}\text { The trained interviewers who conducted the clinician interviews and chart re- } \\
\text { views were not able to be blinded to study group assignment. }\end{array}$ \\
\hline
\end{tabular}

Study adequately protect- Unclear risk

The study was conducted among CCMO and HHCO from the same state. ed against contamination (performance bias)

Other bias High risk Analyses were not adjusted for baseline differences.

\section{Study Design}

Randomised controlled trial

\section{Study Location}

New York city, USA

\section{Country}

High income

\section{Study Period}


Brownell 2006 (Continued)

\section{4 weeks}

\section{Sampling Methods}

Randomly assigned

\section{Data collection method}

Women aged 69 to 83 years (victim) recruited from New York Department for the Aging (DFTA) and its partners.

Pre- and post-intervention data collections were conducted 2 months before and 2 months after interventions. Questionnaires and interviews were conducted. Interviews were conducted for approximately an hour. Data were also collected through audiotapes of each support group sessions.

\section{Inclusion Criteria}

Elderly women self-identified to an aging service provider as having family problems that included family member behaviours associated with physical, psychological, and/or financial abuse; no significant cognitive impairment, based on assessments of professional social workers serving as aging provider referral sources; able to communicate in English; connected to an aging service provider with the capability to provide crisis intervention and additional needed services; and able and willing to attend a weekly support group meeting 2 hours in length, for consecutive weeks.

\section{Ethics and informed consent}

Unclear.

\section{Participants}

\section{Settings}

New York Department for the Aging (DFTA) and its community partners, and Fordham University.

\section{Sample size}

$\mathrm{n}=16$

\section{Participants' characteristics}

Abused older women, identified by participating community partners

\section{Type of Intervention}

Support group program targeted at victims with a structured educational approach.

\section{Start and end date of Intervention}

Not stated

\section{Duration}

8 weeks; 2-hour sessions per week

\section{Aim of Intervention}

To evaluate Elder Mistreatment Psycho-social support group with structured educational component for older women who were victims of mistreatment. The intervention consisted of a 2-hour support group held for 8 consecutive weeks. Curriculum on domestic violence and older women, abuse of older women, legacies of troubled families, enhancing self-esteem, dealing with issues such as depression and anxiety, coping with change in relationships and service resources.

\section{Description of cost and resources}

Not stated.

\section{Evidence of consideration to equity issues}


Brownell 2006 (Continued)

\section{None \\ Information of intervention intensity}

Not stated

\section{Assessment of intensity}

Low

\section{Component of intervention}

Intervention consists of two hours in a psycho-educational support group with sessions held weekly for 8 consecutive weeks. The facilitators were retired professional social workers and graduate social work students.

The educational curriculum included topics such as domestic violence and older women, abuse and neglect of older women, the legacy of troubled families, the silver cord: family history, enhancing selfesteem, depression, anxiety and stress and coping with loss and challenges in the relationships with loved ones, and strategies for change. Video of case studies, group exercise, group activities, discussions and handouts materials were provided. No description was available on control group.

Outcomes

\section{Outcomes}

Primary

None

\section{Secondary}

Health Locus of control

Social support

Depression

Anxiety and somatisation

Self-esteem

Guilt

\section{Measures}

CESD Depression Scale

Rosenberg Self-Esteem Scale

Duke Reliogiosity Scale

Hudson Substance Use Scale

BSI

Hudson Multiproblem symptoms inventory (MPSI)

Social Support Scale

Locus of Control Scale

\section{Time points}

2 measurements were taken; duration not specified

\section{Baseline}

T1: Intervention group, $\mathrm{n}=9$; control group, $\mathrm{n}=6$ 
Brownell 2006 (Continued)

\section{Follow-up}

T2: Intervention group, $\mathrm{n}=$ unclear; control group, $\mathrm{n}=$ unclear

Notes

Authors reported 16 respondents were recruited, however 9 were randomly assigned to intervention group and 6 to the control group. It was unclear if there is one missing respondent.

The study did not find any significant change in outcomes among two groups. Participants were already receiving services from other aging services providers prior to the study.

Sample size was small.

\section{Risk of bias}

\begin{tabular}{lll}
\hline Bias & Authors' judgement & Support for judgement \\
\hline $\begin{array}{l}\text { Random sequence genera- } \\
\text { tion (selection bias) }\end{array}$ & Unclear risk & There was no description on the process of randomisation. \\
\hline $\begin{array}{l}\text { Allocation concealment } \\
\text { (selection bias) }\end{array}$ & Unclear risk & It was not stated if concealment was performed. \\
\hline $\begin{array}{l}\text { Blinding of participants } \\
\text { and personnel (perfor- } \\
\text { mance bias) } \\
\text { All outcomes }\end{array}$ & Unclear risk & It was not stated if blinding was performed. \\
\hline
\end{tabular}

\begin{tabular}{|c|c|c|}
\hline $\begin{array}{l}\text { Blinding of outcome as- } \\
\text { sessment (detection bias) } \\
\text { All outcomes }\end{array}$ & Unclear risk & It was not stated if blinding was performed. \\
\hline
\end{tabular}

\begin{tabular}{ll}
\hline $\begin{array}{l}\text { Incomplete outcome data } \\
\text { (attrition bias) }\end{array}$ & Tow risk \\
All outcomes & otherwise all remaining persons were accounted.
\end{tabular}

Selective reporting (re- High risk One of the outcome measures (self-esteem) was not reported.
porting bias)

\begin{tabular}{lll}
\hline $\begin{array}{l}\text { Baseline outcome mea- } \\
\text { surements similar (selec- } \\
\text { tion bias) }\end{array}$ & High risk & $\begin{array}{l}\text { Authors stated that "Participants did not differ within or between groups on } \\
\text { the identified outcome measures." However, the results suggest that there } \\
\text { were possible differences between the control group and interventions groups } \\
\text { especially alcohol abuse, depression, drug use, and non-physical and physical } \\
\text { abuse. }\end{array}$ \\
\hline $\begin{array}{l}\text { Baseline characteristic } \\
\text { similar (selection bias) }\end{array}$ & Low risk & $\begin{array}{l}\text { Authors stated that there was no statistical differences between the control } \\
\text { and intervention group participants in terms of socio-demographic character- } \\
\text { istic such as age, race and marital status. However, baseline outcomes mea- } \\
\text { surements were not reported in the two groups. }\end{array}$ \\
\hline
\end{tabular}

Knowledge of allocated in- Unclear risk Outcome measurements were collected via face-to-face interview and blinding tervention adequately pre- $\quad$ was not done. It was unclear who assessed the outcome measures.

vented during the study (detection Bias)

\begin{tabular}{|c|c|c|}
\hline $\begin{array}{l}\text { Study adequately protect- } \\
\text { ed against contamination } \\
\text { (performance bias) }\end{array}$ & Unclear risk & $\begin{array}{l}\text { There was no information provided about the control group, thus it is unclear } \\
\text { if participants from the control group were exposed to other aging services } \\
\text { and support groups elsewhere. }\end{array}$ \\
\hline
\end{tabular}

\begin{tabular}{lll}
\hline Other bias $\quad$ High risk $\quad$ The study was conducted as a pilot.
\end{tabular}


There was concern about participants who already had high self-esteem, strong social, relative good health for their age cohort and, self-sufficient as they were attending existing services and volunteered to participate in the study.

\section{Study Design}

Pragmatic randomised parallel group superiority trial.

\section{Study Location}

London and Essex, UK.

\section{Country}

High income

\section{Study Period}

4 Nov 2009 - unspecified date for the extended 24-month follow-up.

\section{Sampling Methods}

Prospective participants recruited by a clinician. The researchers telephoned the carer 24 hours or more after they received the information sheet. To conceal allocation, an online computer-generated randomisation system was used to allocate participants to the intervention or to treatment as usual. Randomisation was stratified by the trust using random permuted blocks. To allow for potential clustering effects in the intervention arm, an allocation ratio of 2:1 (intervention: treatment as usual) was used. A member of the therapy team phoned the participants and informed them of their allocation, either to treatment as usual when they would be contacted for a four-month follow-up or to the intervention when an appointment was made for the therapy to start. Allocation within the individual teams was according to workload.

\section{Data collection method}

Carers were interviewed at baseline and at four and eight months after randomisation, usually in their own home, unless they preferred to come to the research team base.

The outcome assessors were blinded to randomisation status, but not the study participants. Assessors asked participants at the beginning of each interview not to disclose their allocation group.

\section{Inclusion criteria}

Carers of family members referred in the previous year who provided emotional or practical support at least weekly and identified themselves as the primary carer of a family member with dementia not living in 24-hour care; able to give consent, not taking part in other RCT as a carer, living less than 1.5 hours travelling time from researchers' base and not having dementia.

\section{Ethics and informed consent}

Written consent obtained.

Participants Settings

Multicentre (Two mental health community services and one neurological outpatient dementia service).

\section{Sample size}

260 carers 


\section{Participants' characteristics}

$173(67 \%)$ participants were randomised to the intervention group and 87 to treatment as usual. The randomised groups were well-balanced for patient and baseline carer, personal and clinical characteristics except employment status, age, marital status, education level, living status, anxiety score and depression score.

\section{Name of intervention}

STrAtegies for RelaTives (START)

\section{Type of intervention}

Programme to reduce factors influencing elder abuse

\section{Start date of Intervention}

November 2009 to February 2012

\section{Duration}

8-14 weeks

\section{Aim of Intervention}

To determine the clinical effectiveness (measured by the hospital anxiety and depression scale) and cost-effectiveness (reported in an accompanying paper) of eight sessions of a manual-based coping strategy, delivered over 8-14 weeks to carers of family members with dementia, compared with usual service provision, over eight months.

\section{Description of cost and resources}

Not stated

\section{Evidence of consideration to equity issues}

None

\section{Information of intervention intensity}

Not stated

\section{Assessment of intensity}

Medium

\section{Component of intervention}

An individual therapy programme (START, STrAtegies for RelaTives) based on the Coping with Caregiving programme from the United States. The therapy took place where the carers preferred, usually in their homes, without the family member with dementia in the room.

Therapy intervention comprises eight sessions and delivered by supervised psychology graduates to carers of family members with dementia. The programme consisted of psycho-education about dementia, carers' stress, and where to get emotional support; understanding behaviours of the family member being cared for, and behavioural management techniques; changing unhelpful thoughts; promoting acceptance; assertive communication; relaxation; planning for the future; increasing pleasant activities; and maintaining skills learnt. Carers practised these techniques at home, using the manual and relaxation CDs.

\section{Outcomes}

\section{Primary}

Potentially abusive behaviour by the carer. 


\author{
Secondary \\ Non applicable

\section{Measures} \\ Hospital anxiety and depression scale \\ Zarit Burden Interview \\ Modified Conflict Tactics Scale \\ Health status questionnaire \\ Brief COPE \\ Neuropsuchiatric inventory \\ Clinical Dementia rating \\ Quality of life-Alzheimer's disease
}

\title{
Time points
}

Five time points over 24 months period

\section{Baseline}

T1 intervention, $\mathrm{n}=139$; control, $\mathrm{n}=70$

\section{Follow-up}

T2 duration 4 months; intervention, $n=116$; control, $n=56$

T3 duration 8 months; intervention, $\mathrm{n}=99$; control, $\mathrm{n}=52$

T4 duration 12 months; intervention, $\mathrm{n}=87$; control, $\mathrm{n}=46$

T5 duration 24 months; intervention, $\mathrm{n}=84$; control, $\mathrm{n}=40$

Notes $\begin{aligned} & \text { Carers in the intervention group reported less abusive behaviour towards the recipient of care com- } \\ & \text { pared with those in the treatment as usual group at } 8 \text { months (odds ratio } 0.47,95 \% \text { confidence interval } \\ & 0.18 \text { to } 1.23 \text { ), although this was not significant. }\end{aligned}$

\section{Risk of bias}

\begin{tabular}{lll}
\hline Bias & Authors' judgement & Support for judgement \\
\hline $\begin{array}{l}\text { Random sequence genera- } \\
\text { tion (selection bias) }\end{array}$ & Low risk & Randomisation was stratified by trust using random permuted blocks. \\
\hline $\begin{array}{l}\text { Allocation concealment } \\
\text { (selection bias) }\end{array}$ & Low risk & $\begin{array}{l}\text { An online computer-generated randomisation system to allocate participants } \\
\text { to the intervention or to treatment as usual was used to conceal allocation. }\end{array}$ \\
\hline $\begin{array}{l}\text { Blinding of participants } \\
\text { and personnel (perfor- } \\
\text { mance bias) }\end{array}$ & Low risk & $\begin{array}{l}\text { It was not possible to blind the study participants and personnel. However, the } \\
\text { researchers worked in two teams, each assessing outcomes for half the partic- } \\
\text { ipants and providing therapy to those allocated to treatment in the half of par- } \\
\text { ticipants they were not assessing. }\end{array}$ \\
\hline $\begin{array}{l}\text { Blinding of outcome as- } \\
\text { sessment (detection bias) } \\
\text { All outcomes }\end{array}$ & Low risk & $\begin{array}{l}\text { The outcome assessors were blinded to randomisation status. Assessors asked } \\
\text { participants at the beginning of the interview not to disclose their allocation } \\
\text { group. }\end{array}$ \\
\hline
\end{tabular}


Cooper 2015 (Continued)

Incomplete outcome data Low risk (attrition bias)

All outcomes
"Logistic regression were performed to investigate the extent to which missing outcomes varied by baseline characteristics; these were repeated the main analyses adjusting for those factors associated with missingness."

In the control group, $\mathrm{n}=75$ and not $\mathrm{n}=77$ as depicted in the flow chart of participants through study

Selective reporting (re- Low risk $\quad$ All outcomes were reported as intended.
porting bias)
porting bias)

\section{Baseline outcome mea- Low risk} surements similar (selection bias)
There were some differences in baseline characteristics of carer for anxiety scores and total scores on the hospital anxiety and depression scale. "Analyses considered adjustment for imbalances in baseline characteristics between the randomised groups and the differential effects of treatment over time (treatment by time interaction)." Adjustment for baseline differences was performed using logistic regression.
Baseline characteristic Low risk similar (selection bias)
There were some differences in baseline characteristics of carers including employment status, age, education level, marital status and living arrangement with recipient of care. A higher proportion of retired carers, slightly older, living with the recipient of care who were spouses or partners, and with either no school level qualifications or tertiary education in the intervention group.

"Analyses considered adjustment for imbalances in baseline characteristics between the randomised groups and the differential effects of treatment over time (treatment by time interaction)."

Adjustment for baseline differences was performed using logistic regression.

\section{Knowledge of allocated in- Low risk} tervention adequately prevented during the study (detection Bias)

Outcome assessors were blinded to randomisation status, but not it was not possible to blind the study participants. The researchers worked in two teams, each assessing outcomes for approximately half the participants and providing therapy to those allocated to treatment in the half of participants they were not assessing. Assessors asked participants at the beginning of each interview not to disclose their allocation group.

Study adequately protect-

Unclear risk

Randomisation was conducted at the individual level, thus there is a possibility contamination between individuals within the same institution. (performance bias)

Other bias Low risk

The study sample had good external validity except those who consented were, slightly more likely to be married or partnered with the recipient of care than those who did not consent.

All analyses were by intention-to-treat but excluded carers with data missing at both the four- and the eight-month follow-up. Sensitivity analyses were performed to re -analyse the primary outcome and to assess robustness of their conclusions.

\section{Study Design}

Nested randomised controlled trial.

\section{Study Location}

Manhattan, USA 
Davis 2001 (Continued)

\section{Country}

High income

\section{Study Period}

Not stated

\section{Sampling Methods}

All residents from 60 public housing projects were assigned to one of the two levels of public education. Subsequently, 403 residents from these housing projects who reported an incident of elder abuse to the police were randomly assigned to intervention [home visit by the Domestic Violence Intervention and Education Programme Team (DVIEP)] or control group.(usual immediate patrol response by police plus generic DVIEP letter).

\section{Data collection Method}

Mainly telephone interview. If failed to contact victims, home interviews or mail surveys were conducted.

\section{Inclusion Criteria}

Victims aged 55 years and older who called police for elder abuse; between 1/1/1996 to 30/10/1996.

\section{Ethics and informed consent}

Not stated.

Participants

\section{Settings}

Community setting

\section{Sample size}

$n=403$

\section{Participants' characteristics}

Median age = 65 years; predominantly female; $66 \%$ were black and 30\% Latino; $39 \%$ earns \$5,000-9.999 (\$ USA, 1996), $84 \%$ received high school or less; $51 \%$ retired and $28 \%$ on disability scheme, $45 \%$ of the victims lived with the abuser.

About half (49\%) of the trigger incidents involved verbal arguments, others were classified by the police as family disputes (15\%) and misdemeanour offences (9\%), Serious offences include felony (3\%), arrest of the offenders (3\%).

Interventions

Name of Intervention

None

\section{Type of intervention}

Program targeted to victims. A 'blended' mult-strategy intervention of community public education followed by active individual support and monitoring intervention by police and social workers for abused women.

\section{Start and date of Intervention}

Not stated

\section{Duration}

From case to follow-up about one year

\section{Aim of Intervention}


The stated aim is to reduce repeat incidents of elder abuse of those who were abused. Improve victim's awareness, use of resources, victim's knowledge, and willingness to report to the police. One part consisted of public education of presentations provided by the police, posters and leaflets delivered to all elderly persons in the selected communities. The second element consisted of police and social workers following up domestic violence complaints with a home visit and then monitoring of the home.

\section{Description of cost and resources}

Not available

\section{Evidence of consideration to equity issues}

Concentrated low socioeconomic status families, and disadvantaged populations.

\section{Information of intervention intensity}

Not stated.

\section{Assessment of intensity}

High

\section{Component of intervention}

The study had two stages. The first stage involved selected communities, which either received educational materials or did not. The public education consisted of several components, including distributions of leaflets, hanging of poster and community presentation. The second stage involved participants (victims) who either received a home visit from a team or did not. This team consisted of a police officer specialised in domestic violence and a social worker. Four weeks after the initial home visits, the victims received a follow-up telephone call from the counsellor. Batterers were advised that the household was being monitored. Victim was then linked to social services. Controls received the initial patrol response and a generic DVIEP letter containing similar information as in the control group but omitted information on elder abuse and home visits.

Outcomes

Outcomes

\section{Primary}

(1) initial occurrence of elder abuse - report provided counts of cases of elder abuse from communities, but no baseline data are available to calculate change using the follow-up data.

(2) recurrence of elder abuse. Counts for the period provided.

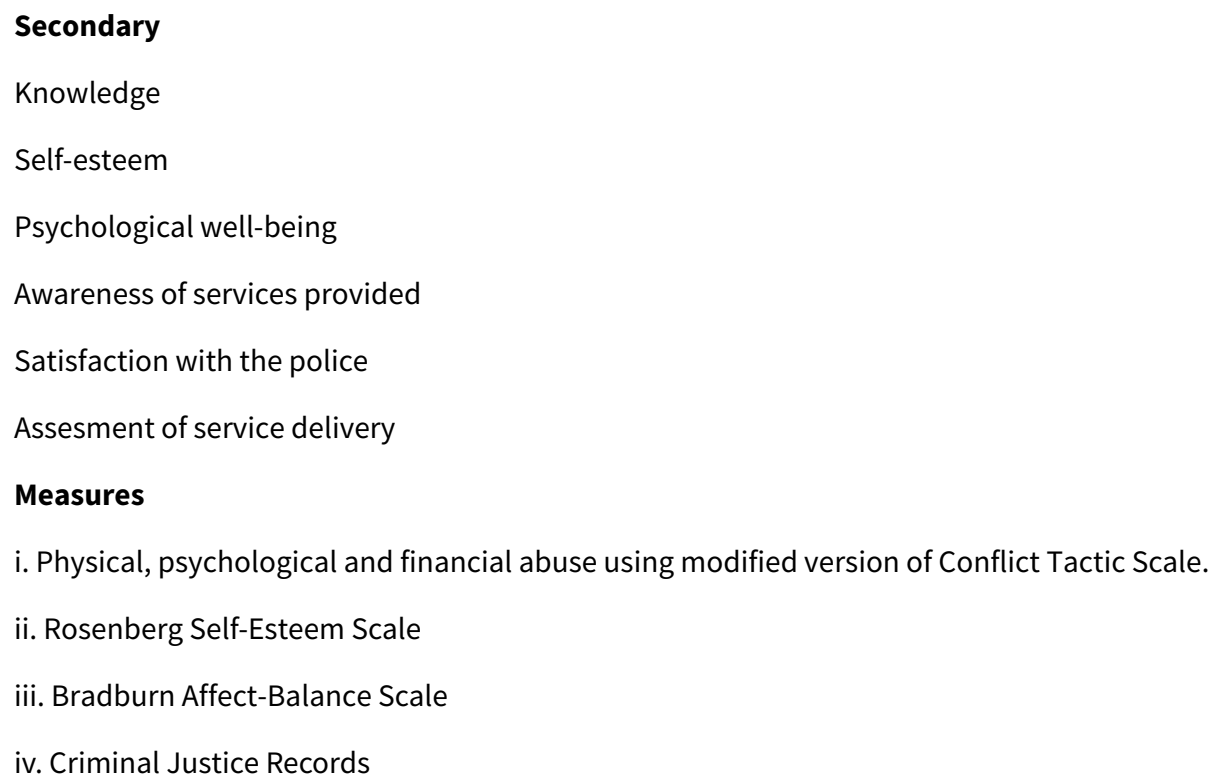


Davis 2001 (Continued)

\section{Time points}

Three time points; 6 weeks, 6 months and 12 months after the trigger incident.

\section{Baseline}

$n=403$; no information provided on the numbers in the control or intervention groups

\section{Follow-up}

Follow-up is first interview 6 weeks after intake, "2nd interview after 6 months, 3rd interview 12 months after 2nd interview".

Intervention targeted at elderly aged 55 years and above. The median age of participants was 65 years. The study was aimed at elders, although the police screened the files for victims from 55 years of age. The range of ages was not included by the investigators, and given the intent of the intervention was elders, and the mean age was 65 , we chose to include this study.

Duration of intervention was unclear, particular frequency of educational materials provided or duration spent for "one home visit".

Participants who only received the public education intervention did not differ from those in the control group.

Participants in the home visit group fared worse than those in the control group.

Those who were in both intervention group suffer more repeated victimisation.

Outcomes were poorly reported.

There were cases where victims assigned to the intended treatment did not receive treatment, or vice versa.

Interventions lead to more abuse reported - authors hypothesised that the abusers of the elderly may have become angered by attempts to intervene.

\section{Risk of bias}

Bias Authors' judgement Support for judgement

Random sequence genera- High risk tion (selection bias)
No elaboration on randomisation. " Four hundred three residents of public housing who reported an incident of elder abuse to the police were randomly assigned to receive or not receive two interventions designed to reduce the incidence of repeat abuse." The authors stated that the randomisation did not work.

\begin{tabular}{lll}
\hline $\begin{array}{l}\text { Allocation concealment } \\
\text { (selection bias) }\end{array}$ & Unclear risk & Not stated. \\
\hline $\begin{array}{l}\text { Blinding of participants } \\
\begin{array}{l}\text { and personnel (perfor- } \\
\text { mance bias) }\end{array}\end{array}$ & High risk & $\begin{array}{l}\text { It was not stated if blinding was performed. However, it is likely that blinding } \\
\text { was not possible as participants were aware if they received a visit from the } \\
\text { tll outcomes }\end{array}$ \\
\hline
\end{tabular}
sessment (detection bias) All outcomes

$\begin{array}{lll}\begin{array}{l}\text { Incomplete outcome data } \\ \text { (attrition bias) }\end{array} & \text { High risk } & \begin{array}{l}\text { Interview on non-completion varied significantly by treatment group. Dropout } \\ \text { rates in the control group (from } 35.1 \% \text { to } 39.7 \%) \text { were significantly higher than } \\ \text { All outcomes }\end{array} \\ \text { the home visit group ( } 25.8 \% \text { to } 29.7 \%) \text {, depending on the time points. }\end{array}$


Davis 2001 (Continued)

Selective reporting (re- Low risk $\quad$ All expected outcomes reported.
porting bias)

porting bias)

Baseline outcome mea-

Unclear risk

Not stated.

surements similar (selec-

tion bias)

\begin{tabular}{ll}
\hline $\begin{array}{l}\text { Baseline characteristic } \\
\text { similar (selection bias) }\end{array}$ & Unclear risk
\end{tabular}

\begin{tabular}{ll}
\hline $\begin{array}{l}\text { Knowledge of allocated in- } \\
\text { tervention adequately pre- } \\
\begin{array}{l}\text { vented during the study } \\
\text { (detection Bias) }\end{array}\end{array}$ & $\begin{array}{l}\text { For the primary outcome, objective measurements were collected from the } \\
\text { Criminal Justice Records. But for the secondary outcome it is unclear as to } \\
\text { who were the assessors in this study. }\end{array}$ \\
\hline
\end{tabular}

(detection Bias)

Study adequately protect- High risk $\quad$ Cross-over of participants occurred during the study.
ed against contamination
(performance bias)

\begin{tabular}{ll}
\hline Other bias $\quad$ Low risk & $\begin{array}{l}\text { Cox analysis was performed to adjust for differences in the two groups. Objec- } \\
\text { tive and validated measurements were used. }\end{array}$ \\
\hline
\end{tabular}

Hsieh 2009

Methods

Study Design

Controlled before-and-after (CBA) study

Study Location

Southern Taiwan

\section{Country}

High income

\section{Study Period}

6 months (January- June 2008)

\section{Sampling Methods}

Facility control sampling

\section{Data collection method}

Data collected from self-administered questionnaire. Participants recruited from four officially registered nursing homes located in southern Taiwan.

\section{Inclusion Criteria}

i) Taiwanese

ii) 20 years and above

iii) had not participated in similar activity in prior 2 months

iv) caregivers selected based on Caregiver Psychological Elder Abuse Behavior Scale.

Ethics and informed consent 


\section{Settings}

Nursing home

\section{Sample size}

$\mathrm{n}=112$ caregivers (recruited)

\section{Participants characteristic}

The majority of the participants were female (97\%), married (77\%), employed as nurse aids (79\%); with a mean age of 42.9 years ( $S D=9.5)$, the youngest being 21 and the oldest being 60 years old. Average years of education completed was 10.8 years $(S D=5.3)$, with the lowest being 6 and the highest being 16 years. The average length of employment at their current position was 69.7 months $(S D=54.1)$. No participants had previously attended a support group, although $75 \%$ had received training in geriatric care prior to their current position. Most of participants reported a 30-min break during their work shift $(76 \%)$, mean hours of daily work was $9.6(\mathrm{SD}=2.0$, range $=6-12)$ and their monthly salary was between NT\$16,500 and 54,000 dollars ( $M=25,901, S D=7753)$

Interventions

\section{Name of Intervention}

Not stated

\section{Type of intervention}

Educational intervention

\section{Start and end date of Intervention}

Within the duration of study period

\section{Duration}

8 weeks

\section{Aim of Intervention}

Aimed at decreasing the caregiver's inappropriate verbal or emotional behaviours, improve ability to cope with stress, promote knowledge, and providing geriatric care.

\section{Description of cost and resources}

Not stated

\section{Evidence of consideration to equity issues}

Not stated

\section{Information of intervention intensity}

"Caregivers" involved, reinforcement of training

\section{Assessment of intensity}

Low

\section{Component of intervention}

Intervention group was exposed to intervention which consisted of 90-minute teaching sessions weekly for 8 consecutive weeks designed by research team. A trained graduate nurse serve as group facilitator. The program covered content of aging and associated problems related to managing residents' health problems, institutional management, dealing with stressful caregiving context, and obtaining personal resources. For each session, the lecture topic was given 30 for minutes, the following 40 minutes al- 
Hsieh 2009 (Continued)

lowed for free sharing and mutual support among group members and last 20 minutes for integrative discussion. The control group did not receive any extra intervention.

\begin{tabular}{|c|c|c|}
\hline Outcomes & $\begin{array}{l}\text { Outcomes } \\
\text { Primary } \\
\text { Caregivers' psycholog } \\
\text { Secondary } \\
\text { Non applicable } \\
\text { Measures } \\
\text { Caregiver Psychologic } \\
\text { The Work Stressors In } \\
\text { Knowledge of Geronto } \\
\text { Time points } \\
\text { 2 measurements; } 1 \text { we } \\
\text { 1) Baseline } \\
\text { n = } 112 \\
\text { 2) Follow-up } \\
n=100\end{array}$ & $\begin{array}{l}\text { Cal abusive behaviour } \\
\text { entory (WSI) } \\
\text { ogy Nursing Scale (KGNS) } \\
\text { Erior intervention; and } 1 \text { week following the intervention }\end{array}$ \\
\hline Notes & \multicolumn{2}{|c|}{ Improvement in knowledge and behaviour, but not work-related stress. } \\
\hline \multicolumn{3}{|l|}{ Risk of bias } \\
\hline Bias & Authors' judgement & Support for judgement \\
\hline $\begin{array}{l}\text { Random sequence genera- } \\
\text { tion (selection bias) }\end{array}$ & High risk & $\begin{array}{l}\text { Not applicable as this is a CBA study. } \\
\text { No randomisation involved. All caregivers in the selected institutions are ex- } \\
\text { posed to either intervention or control. }\end{array}$ \\
\hline $\begin{array}{l}\text { Allocation concealment } \\
\text { (selection bias) }\end{array}$ & High risk & Not applicable as this is a CBA study. \\
\hline $\begin{array}{l}\text { Blinding of participants } \\
\text { and personnel (perfor- } \\
\text { mance bias) } \\
\text { All outcomes }\end{array}$ & High risk & Not applicable as this is a CBA study. \\
\hline
\end{tabular}

\begin{tabular}{|c|c|c|}
\hline $\begin{array}{l}\text { Blinding of outcome as- } \\
\text { sessment (detection bias) } \\
\text { All outcomes }\end{array}$ & High risk & Not applicable as this is a CBA study. \\
\hline $\begin{array}{l}\text { Incomplete outcome data } \\
\text { (attrition bias) } \\
\text { All outcomes }\end{array}$ & Low risk & $\begin{array}{l}112 \text { caregivers were recruited however only those who completed at least six } \\
\text { of eight sessions were included in the analysis, } n=100 \text { ( } 50 \text { in each group). }\end{array}$ \\
\hline $\begin{array}{l}\text { Selective reporting (re- } \\
\text { porting bias) }\end{array}$ & Low risk & Expected outcomes reported. \\
\hline
\end{tabular}


Hsieh 2009 (Continued) surements similar (selec-

Low risk tion bias)
The baseline outcome measurements show that caregivers in the intervention group have higher tendency towards abusive behaviour, more knowledgeable towards elderly care and had higher work stressors compared to the control group.

\section{Baseline characteristic Low risk} similar (selection bias)

Participants characteristics similar except for age and monthly salary.

Participants in intervention group were slightly younger and earned more than the control group.

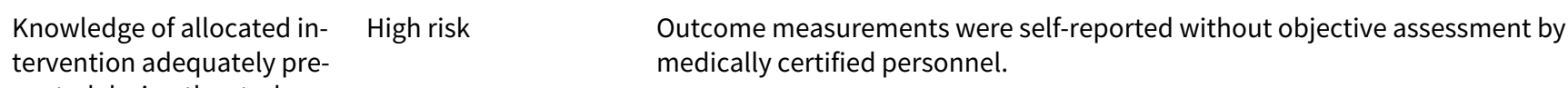
vented during the study medically certified personnel.

(detection Bias)

\begin{tabular}{|c|c|c|}
\hline $\begin{array}{l}\text { Study adequately protect- } \\
\text { ed against contamination } \\
\text { (performance bias) }\end{array}$ & Low risk & $\begin{array}{l}\text { Between-institutional control was used to avoid participant contamination, } \\
\text { that is, two facilities served as experimental sites and two served as control } \\
\text { sites. }\end{array}$ \\
\hline
\end{tabular}

Other bias Low risk The final result are adjusted for baseline measurement. "Between group differences or group effects were measured by General Linear Model (GLM) univariate analyses, adjusting for baseline and monthly salary while within group differences were measured by paired t-tests"

The self-administered measurement tools were validated before the study.

\section{Richardson 2002}

\section{Methods}

\section{Study Design}

Randomised controlled trial

\section{Study Location}

Livingston County, North London, United Kingdom

\section{Country}

High income

\section{Study Period}

August, 1999 to August, 2000

\section{Sampling Methods}

Individuals were randomly assigned using computed-generated numbers to either intervention or control group. The randomisation was concealed until intervention was allocated.

\section{Data collection method}

Self-report questionnaire pre- and post-interventions

\section{Inclusion criteria}

Ethics and informed consent

Ehical approval obtained from "The Local Ethics Committee" and informed consent obtained.

Participants Settings


Richardson 2002 (Continued)

Institution

\section{Sample size}

$\mathrm{n}=86$

\section{Participants characteristic}

Participants were health personnel employed by local community health trust of social service worked with older people and hat not yet attended a course on managing abuse of older people. They comprised nursing staff, care assistants, care managers and social workers. Intervention group (Group 1) had $n=31$ while control group (Group 2) had $n=33$. There was no significant difference between intervention and control groups in relation to socio-demographic profile, and attitude. In group 1, 77.4\% was female and in group 2, 81.8\%. Both groups mainly consisted of care assistants; (Group 1-61.3\%; Group 2 - 45.5\%). Mean years of experience was longer in Group 2 (13.7 years; SD $=8.5$ ) compared to Group 1 (11.1; SD 10). Mean attitude scores in group 1 and group 2 were $9.5(S D=6.5)$ and $8.8(S D=5.9)$, respectively. There was a statistically significant difference in the mean KAMA score where the intervention group was lower (Mean 22.3; range $=12.1$ to 51.8; $\mathrm{SD}=9.6$ than control group (Mean 28.5; range = 8.9 to $58.9 ; \mathrm{SD}=13.3$ ).

\section{Name of Intervention}

Educational intervention

\section{Start date of Intervention}

October, 1999 (10 months)

\section{Duration}

Each workshop was 1 day in duration

\section{Aim of Intervention}

To compare the effectiveness of attending an educational course (Group 1) to printed educational material (Group 2) in improving management of abuse of older people.

\section{Description of cost and resources}

Not stated.

\section{Evidence of consideration to equity issues}

None.

\section{Information of intervention intensity}

Not stated.

\section{Assessment of intensity}

Low.

\section{Component of intervention}

Group 1 attended an educational course commissioned by the employing NHS trust and social services. This educational course lasted for an hour. Those in Group 2 were given reading material with the same content as the course. The programmes targeted identification and management of all types of abuse i.e. neglect, verbal, physical and financial abuse. They were based on the policy, practice guidance and procedures on responding to abuse and inadequate care of vulnerable adults which was operational in both health and social services. Course duration and frequency were not stated.

Outcomes

\section{Outcomes}

Primary 
Richardson 2002 (Continued)

None

\section{Secondary}

Knowledge and management of elder abuse

\section{Measures}

Knowledge and management questionnaire (KAMA)

Maslach Burnout Inventory (MBI)

\section{Time points}

2 measurements were taken. Duration not specified

\section{Baseline}

87 people approached by the researchers, one refused to participate. The baseline assessment was completed by 79 participants, of whom 7 refused the post-intervention assessment.

\section{Follow-up}

Follow-up data were obtained on 64 (81\%) of those who consented to take part.

Notes There were no significant differences between non-participants and non-completers.

\section{Risk of bias}

\begin{tabular}{lll}
\hline Bias & Authors' judgement & Support for judgement \\
\hline $\begin{array}{l}\text { Random sequence genera- } \\
\text { tion (selection bias) }\end{array}$ & Low risk & $\begin{array}{l}\text { "All eligible participants were randomised using computer-generated numbers } \\
\text { into either Group 1 or 2" }\end{array}$ \\
\hline $\begin{array}{l}\text { Allocation concealment } \\
\text { (selection bias) }\end{array}$ & Low risk & $\begin{array}{l}\text { "The randomization was concealed until the intervention was allocated". "The } \\
\text { group allocation were disclosed after scoring was completed." }\end{array}$ \\
\hline $\begin{array}{l}\text { Blinding of participants } \\
\begin{array}{l}\text { and personnel (perfor- } \\
\text { mance bias) }\end{array}\end{array}$ & High risk & $\begin{array}{l}\text { "The participants unavoidably know to which arm of the trial they had been al- } \\
\text { located." }\end{array}$ \\
\end{tabular}

\begin{tabular}{ll}
\hline $\begin{array}{l}\text { Blinding of outcome as- } \\
\text { sessment (detection bias) }\end{array}$ & Low risk
\end{tabular}$\quad \begin{aligned} & \text { "The raters were blinded to the allocation as there was no indication on the } \\
& \text { completed assessment either concerning the identity of the participants or } \\
& \text { which group they had been randomised to or whether in instruments being } \\
& \text { scored as pre or post intervention." }\end{aligned}$

\begin{tabular}{|c|c|c|}
\hline $\begin{array}{l}\text { Incomplete outcome data } \\
\text { (attrition bias) } \\
\text { All outcomes }\end{array}$ & Low risk & $\begin{array}{l}\text { There were no significant differences in the characteristics of participants } \\
\text { compared to non-participants and non-completers. }\end{array}$ \\
\hline
\end{tabular}

\begin{tabular}{lll}
\hline $\begin{array}{l}\text { Selective reporting (re- } \\
\text { porting bias) }\end{array}$ & Low risk & All measurements outcomes were reported as intended. \\
\hline $\begin{array}{l}\text { Baseline outcome mea- } \\
\text { surements similar (selec- } \\
\text { tion bias) }\end{array}$ & High risk & $\begin{array}{l}\text { "There was a significant difference at baseline in KAMA scores }(P<0.05) \text { with } \\
\text { those randomised to Group } 2 \text { scoring higher." }\end{array}$ \\
\hline
\end{tabular}

\begin{tabular}{ll}
\hline $\begin{array}{l}\text { Baseline characteristic } \\
\text { similar (selection bias) }\end{array} \quad$ Low risk & $\begin{array}{l}\text { There were no significant differences in those randomised to either inter- } \\
\text { vention group in terms of gender, years of experience, professional status, }\end{array}$
\end{tabular}


Knowledge of allocated in- Low risk tervention adequately prevented during the study (detection Bias)
The participants unavoidably knew to which arm of the trial they had been allocated. The tutor of those in Group 1 was blinded to who was participating in the study. The raters were blind to the allocation, as there was no indication on the completed assessment either concerning the identity of the participants or which group they had been randomised to or whether the instrument being scored was pre- or post-intervention. The group allocations were disclosed after scoring was completed.

\begin{tabular}{|c|c|c|}
\hline $\begin{array}{l}\text { Study adequately protect- } \\
\text { ed against contamination } \\
\text { (performance bias) }\end{array}$ & Unclear risk & $\begin{array}{l}\text { The participants worked in the same locality. There is possibility of the partici } \\
\text { pants from different arms meeting each other. }\end{array}$ \\
\hline Other bias & Low risk & $\begin{array}{l}\text { Analyses based on per protocol and not by intention-to-treat. } \\
\text { However, all research tools were validated and logistic regression modelling } \\
\text { was used to adjust for group differences at baseline. }\end{array}$ \\
\hline
\end{tabular}

Teresi 2013

Methods

\section{Study Design}

Cluster-randomised trial

\section{Study Location}

New York City, USA

\section{Country}

High Income

\section{Study Period}

July 2008 to December 2011

\section{Sampling Methods}

This study was conducted in five large nursing homes. A total of 54 units ( 47 long- and 7 short-stay) at the five sites sampled. Short-stay units were excluded from these analyses; thus the number of units was cluster randomised into 23 in the experimental and 24 in the control groups. Staff in the experimental units received the training and implementation protocols, whereas individuals in the comparison units did not. However, staff on the comparison units did receive training on the reporting form used to collect recognition outcome data.

\section{Data collection method}

Data used to evaluate impact of this training intervention for nursing staff on knowledge, recognition and reporting of resident-to-resident elder mistreatment were collected through medical chart reviews. Elderly persons completed a 10 -item self-report instrument.

\section{Inclusion criteria}

Long-term stay residents and not receiving hospice care.

\section{Ethics and informed consent}

Ehics obtained from Weill Cornell Medical Center (U.S. National Institute on Aging, NIA) and Research Division of the Hebrew Home at Riverdale (U.S. NIA). 
Teresi 2013 (Continued)

Participants

\section{Settings}

New York City nursing home units

\section{Sample size}

A sample of 1405 residents ( 685 in the control and 720 in the intervention group) from 47 New York City nursing home units ( 23 experimental and 24 control) in 5 nursing homes were assessed. A total of 325 Certified Nursing Assistant (CNA) were trained on Module 1; 317 CNAs attended the sessions on Module 2 and 322 CNAs were trained on the implementation and use of the R-REM BRDS (incident tracking sheets).

\section{Participants characteristic}

None stated for the CNA group.

As for the resident characteristics at baseline, both resident groups (control and experimental) were primarily female, white, and widowed. The control and the experimental group residents were of equivalent age. There were no significant demographic differences between the two groups.

Interventions

\section{Name of intervention}

Resident-to-resident elder mistreatment (R-REM)

\section{Type of intervention}

Programme to increase detection rate for prevention of elder abuse

\section{Start date of Intervention}

July 2008 to December 2011.

\section{Duration}

Four weeks with a follow-up of 6 and 12 months.

\section{Aim of Intervention}

To evaluate the impact of a newly developed training intervention for nursing staff on knowledge, recognition and reporting of resident-to-resident elder mistreatment.

\section{Description of cost and resources}

Not stated

Evidence of consideration to equity issues

None

\section{Information of intervention intensity}

Not stated

\section{Assessment of intensity}

High

\section{Component of intervention}

The intervention involved training of certified nursing assistants on R-REM in three distinct modules: 1) Recognition and Risk Factors, 2) Management, and 3) Implementation of best practices related to RREM.

The trainers were experienced professionals with backgrounds in sociology, nursing and nursing home staff education, social work, public health and nursing home administration. Each session was scheduled twice for all the nursing shifts, including the night staff. Multiple time periods training was con- 
Teresi 2013 (Continued)

ducted to ensure that almost all nursing assistants were able to attend. Training was delivered in a multimodality format including experiential service training, presentation of a film, reviewing practice sheet.

\begin{tabular}{|c|c|c|}
\hline \multirow[t]{19}{*}{ Outcomes } & \multirow{2}{*}{\multicolumn{2}{|c|}{$\begin{array}{l}\text { Outcomes } \\
\text { Primary }\end{array}$}} \\
\hline & & \\
\hline & \multicolumn{2}{|c|}{ Reporting of resident-to-resident elder mistreatment among nursing staff } \\
\hline & \multicolumn{2}{|c|}{ knowledge, and recognition of R-REM among nursing staff } \\
\hline & \multicolumn{2}{|l|}{ Measures } \\
\hline & \multicolumn{2}{|c|}{ R-REM behavior recognition and documentation sheet (BRDS)- self-report by nursing staff } \\
\hline & \multicolumn{2}{|c|}{$\begin{array}{l}\text { R-REM knowledge tests: Recognising R-REM (Module 1) and R-REM Management (Module 2)- self- report } \\
\text { by nursing staff }\end{array}$} \\
\hline & \multicolumn{2}{|c|}{ R-REM staff instrument - interview } \\
\hline & \multicolumn{2}{|c|}{ The Care Diagnostic Scale (CAREDIAG) } \\
\hline & \multicolumn{2}{|l|}{ Time points } \\
\hline & \multicolumn{2}{|l|}{ Three time points. } \\
\hline & \multicolumn{2}{|c|}{ Data for knowledge were collected at baseline, pre and post training. } \\
\hline & \multicolumn{2}{|c|}{ Data for recognition and documentation of R-REM were collected on a weekly basis } \\
\hline & \multicolumn{2}{|c|}{ Interview data from residents and staff were collected at three time points: baseline, 6 and 12 months. } \\
\hline & \multicolumn{2}{|l|}{ Baseline } \\
\hline & \multicolumn{2}{|c|}{ T1: baseline ( $n=23$ experimental group and $n=24$ control) } \\
\hline & \multicolumn{2}{|l|}{ Follow-up } \\
\hline & \multicolumn{2}{|c|}{ T2: 6 months follow-up } \\
\hline & \multicolumn{2}{|c|}{ T3: 12 months follow-up } \\
\hline \multirow[t]{3}{*}{ Notes } & \multicolumn{2}{|c|}{ Minimal information was provided for the certified nursing assistant (CNA) } \\
\hline & \multicolumn{2}{|c|}{ There was 12 CNAs who switched from the control group to intervention group. } \\
\hline & \multicolumn{2}{|c|}{ The training intervention was effective in enhancing knowledge, recognition and reporting of R-REM. } \\
\hline \multicolumn{3}{|l|}{ Risk of bias } \\
\hline Bias & Authors' judgement & Support for judgement \\
\hline $\begin{array}{l}\text { Random sequence genera- } \\
\text { tion (selection bias) }\end{array}$ & Low risk & $\begin{array}{l}\text { Randomisation of experimental (intervention) units and matched comparison } \\
\text { (control) units within facilities, using the SPSS pseudo-random number gener- } \\
\text { ator (StatisticalPackage for the Social Sciences, version 18) procedure, }\end{array}$ \\
\hline $\begin{array}{l}\text { Allocation concealment } \\
\text { (selection bias) }\end{array}$ & Unclear risk & Not stated. \\
\hline
\end{tabular}


Teresi 2013 (Continued)

Blinding of participants Low risk_ "All project staff except the project director and statistical staff responsible for and personnel (perforrandomization were blinded regarding the intervention. This was possible bemance bias)

All outcomes cause all baseline interviews were collected prior to the trainers delivering the intervention."

However, it was not possible to blind the participants.

\begin{tabular}{|c|c|c|}
\hline $\begin{array}{l}\text { Blinding of outcome as- } \\
\text { sessment (detection bias) } \\
\text { All outcomes }\end{array}$ & Low risk & $\begin{array}{l}\text { "Evaluation interviewers were primarily nursing or pre-medical students or } \\
\text { post BA/BS and graduate school students." Assessments were conducted not } \\
\text { by the trainers, and all project staff were blinded. }\end{array}$ \\
\hline
\end{tabular}

All outcomes by the trainers, and all project staff were blinded.

Incomplete outcome data Low risk

(attrition bias)

All outcomes
There was differential attrition between groups, despite this differential attrition, the groups remained balanced on nearly all variables. Furthermore, these differences were addressed statistically and in sensitivity analyses.

Selective reporting (re- Low risk $\quad$ All outcomes were reported as intended.
porting bias)

\begin{tabular}{|c|c|c|}
\hline $\begin{array}{l}\text { Baseline outcome mea- } \\
\text { surements similar (selec- } \\
\text { tion bias) }\end{array}$ & Low risk & $\begin{array}{l}\text { There was imbalance between the intervention and comparison groups of the } \\
\text { residents at baseline characteristics, i.e., functional and cognitive status. How- } \\
\text { ever adjustments were made to control for these differences. }\end{array}$ \\
\hline
\end{tabular}

\begin{tabular}{lll}
\hline $\begin{array}{l}\text { Baseline characteristic } \\
\text { similar (selection bias) }\end{array}$ & Unclear risk & $\begin{array}{l}\text { No information ws given on the Certified Nursing Assistants (CNA). There was } \\
\text { no difference in the intervention and comparison groups of the residents' } \\
\text { baseline characteristics. }\end{array}$ \\
\hline $\begin{array}{l}\text { Knowledge of allocated in- } \\
\text { tervention adequately pre- } \\
\begin{array}{l}\text { vented during the study } \\
\text { (detection Bias) }\end{array}\end{array}$ & High risk & $\begin{array}{l}\text { Outcome assessors were not blinded to randomisation status. It was also not } \\
\text { possible to blind the CNAs. }\end{array}$ \\
\hline $\begin{array}{l}\text { Study adequately protect- } \\
\text { ed against contamination } \\
\text { (performance bias) }\end{array}$ & High risk & $\begin{array}{l}\text { Randomisation was of units was within facilities, thus there is the potential for } \\
\text { contamination. }\end{array}$ \\
\hline \begin{tabular}{l} 
Other bias \\
\hline
\end{tabular} & Unclear risk & Baseline characteristics of the CNAs were not reported. \\
\hline
\end{tabular}

Characteristics of excluded studies [ordered by study ID]

\begin{tabular}{|c|c|}
\hline Study & Reason for exclusion \\
\hline Acierno 2004 & Ineligible study design - pre and post intervention with no control, 2 measurement points \\
\hline ACT government 2006 & No evaluation reported \\
\hline Action on Elder Abuse 1995 & Ineligible study design - survey \\
\hline Action on Elder Abuse 2006 & Ineligible study design - case study \\
\hline Aday 1991 & Intervention not aimed at elder abuse, no abuse-related outcomes \\
\hline Aday 1993 & Intervention not aimed at elder abuse, no abuse-related outcomes \\
\hline $\begin{array}{l}\text { Age Concern New Zealand } \\
2005\end{array}$ & Ineligible study design - descriptive secondary data \\
\hline
\end{tabular}




\begin{tabular}{|c|c|}
\hline Study & Reason for exclusion \\
\hline Anetzberger 1993 & Ineligible study design - key informant interviews \\
\hline Anetzberger 2000b & Ineligible study design - case study \\
\hline Anetzberger 2010 & No abuse-related outcomes \\
\hline Anetzerberger 2000a & Ineligible study design - descriptive/protocol, no quantitative outcomes reported \\
\hline Antonio 2009 & Ineligible study design - survey and qualitative interviews \\
\hline Ash 2013 & Ineligible study design - qualitative interview, focus groups, direct observation \\
\hline Austin 2005 & Ineligible study design - qualitative interview, focus groups \\
\hline Belle 2006 & Intervention not aimed at elder abuse, no abuse-related outcomes \\
\hline Ben Natan 2013 & No evaluation reported \\
\hline Bennett 1993 & Ineligible study design - descriptive \\
\hline Bennett 2000 & Ineligible study design - descriptive \\
\hline Blakely 2001 & Ineligible study design - survey \\
\hline Bomba 2006 & No assessment, no outcome reported \\
\hline Bowland 2012 & $\begin{array}{l}\text { Ineligible population (women aged } 55 \text { years or older who previously suffered from child abuse, sex- } \\
\text { ual assault or domestic violence) }\end{array}$ \\
\hline Braun 1997 & Ineligible study design - pre and post intervention with no control, 2 measurement points \\
\hline Braun 2004 & Ineligible study design - descriptive \\
\hline Breen 2009 & Ineligible study design - qualitative analysis, no outcome reported \\
\hline Brown 1992 & No abuse-related outcomes \\
\hline Brown 1998 & Ineligible study design - descriptive case reviews \\
\hline Brown 2004 & Editorial \\
\hline Brownell 2003 & Ineligible study design- observational analytical study \\
\hline Butler 2008 & $\begin{array}{l}\text { Ineligible study design - pre and post intervention with no control, } 2 \text { measurement points, no } \\
\text { abuse-related outcomes }\end{array}$ \\
\hline Cambridge 2006 & Ineligible study design - qualitative interviews \\
\hline Cambridge 2011 & Ineligible study design - descriptive secondary data \\
\hline Chaffin 2002 & Ineligible study design - survey \\
\hline Chang 2003 & Ineligible study design - qualitative interviews \\
\hline Clancy 2011 & Ineligible study design - cross-sectional with 2 time points (baseline and 6 months) \\
\hline
\end{tabular}




\begin{tabular}{|c|c|}
\hline Study & Reason for exclusion \\
\hline Cook-Daniels 2004 & Ineligible study design - survey \\
\hline Coon 2003 & Intervention not aimed at elder abuse, no abuse-related outcomes \\
\hline Cooper 2012 & $\begin{array}{l}\text { Ineligible design, uncontrolled before-and-after study; not an interrupted time series analysis as } \\
\text { originally thought as insufficient pre-intervention measurement }\end{array}$ \\
\hline Cummings 2002 & Intervention not aimed at elder abuse, no abuse-related outcomes \\
\hline Cupitt 1997 & Ineligible study design - cross-sectional survey, small sample size - 10 \\
\hline Daly 2003 & Ineligible study design - cross-sectional \\
\hline Daly 2011a & Ineligible study design - case studies \\
\hline Daniels 1989 & Ineligible study design - survey \\
\hline Dauenhauer 2007 & Ineligible study design - cross-sectional survey \\
\hline Davies 2011 & Ineligible study design - observational study only \\
\hline Day 2010 & No outcomes reported \\
\hline Dellmann-Jenkins 1991 & Intervention not aimed at elder abuse, no abuse-related outcomes \\
\hline Desy 2008 & Ineligible study design - pre and post intervention with no control, 3 measurement points \\
\hline Dillenburger 2008 & Ineligible population (victims of community violence) \\
\hline Dolon 1989 & Ineligible study design - cross-sectional survey \\
\hline Dorfman 2003 & $\begin{array}{l}\text { Ineligible study design - pre and post intervention with no control, } 2 \text { measurement points, no } \\
\text { abuse-related outcomes }\end{array}$ \\
\hline Drossel 2011 & Intervention not aimed at elder abuse, no abuse-related outcomes \\
\hline Eisdorfer 2003 & No abuse-related outcomes \\
\hline Ejaz 2001 & Ineligible study design, evaluation period short (8 weeks) \\
\hline Elliott 2010 & No abuse-related outcomes \\
\hline Ernst 2012 & Ineligible study design - retrospective secondary data \\
\hline Everson 1996 & Ineligible study design - training program only \\
\hline Feldt 1992 & $\begin{array}{l}\text { Ineligible study design - pre and post intervention with no control, } 2 \\
\text { measurement points, no abuse related outcomes }\end{array}$ \\
\hline Fiegener 1989 & Ineligible study design - survey \\
\hline Filinson 1993 & Ineligible study design - post intervention with matched control, 2 measurement points \\
\hline Filinson 2001 & Ineligible study design - survey and focus groups, no-abuse related outcomes \\
\hline
\end{tabular}




\begin{tabular}{|c|c|}
\hline Study & Reason for exclusion \\
\hline Filinson 2006 & Ineligible study design - review secondary resources \\
\hline Finkel 2007 & No abuse-related outcomes \\
\hline Fisher 2003 & Ineligible study design - survey \\
\hline Fisher 2003a & No outcomes reported \\
\hline Foelker 1990 & Ineligible study design - descriptive \\
\hline Fox 1996 & No abuse-related outcomes \\
\hline Fujiwara 2006 & No abuse-related outcomes \\
\hline Fulmer 1985 & No intervention \\
\hline Gallagher-Thompson 2007 & No abuse-related outcomes \\
\hline Gassoumis 2015 & Ineligible study design - retrospective case control \\
\hline Georgia 1999 & Ineligible study design - case series \\
\hline Georgia 1999a & Ineligible study design - secondary data analysis \\
\hline Gillum 2009 & Not abuse of the elderly \\
\hline Gironda 2010 & Reported aggregate outcomes on programme activity and quality \\
\hline Gittler 2008 & Ineligible study design - narrative \\
\hline Gold 1989 & Ineligible study design - post intervention evaluation, quality of programme evaluation \\
\hline Goldberg 2001 & Ineligible study design - qualitative interviews \\
\hline Goodrich 1997 & Ineligible study design - survey \\
\hline Goodridge 1997 & $\begin{array}{l}\text { Ineligible study design - pre and post study with no control, } 2 \text { measurement with post measure- } \\
\text { ment } 7-8 \text { weeks after program }\end{array}$ \\
\hline Gorgen 2005 & Ineligible study design - descriptive \\
\hline Griffith 2009 & Ineligible study design - descriptive \\
\hline Groh 2011 & No outcomes reported \\
\hline Hagen 1995 & No evaluation \\
\hline Hannon 2008 & Intervention not aimed at elder abuse, no abuse-related outcomes \\
\hline Hanratty 2011 & $\begin{array}{l}\text { Ineligible study design - matched comparison of housing participants and non participants, no } \\
\text { abuse-related outcomes }\end{array}$ \\
\hline Harmer-Beem 2005 & $\begin{array}{l}\text { Ineligible study design - pre post study with no control, } 2 \text { measurements with baseline and immedi- } \\
\text { ate post measurement }\end{array}$ \\
\hline
\end{tabular}




\begin{tabular}{|c|c|}
\hline Study & Reason for exclusion \\
\hline Harries 2014 & Immediate assessment only \\
\hline Harry 2000 & Ineligible study design - qualitative focus groups \\
\hline Hawes 2010 & Ineligible study design - survey and focus groups \\
\hline Heath 2002 & Ineligible study design - retrospective cohort \\
\hline Heath 2005 & Ineligible study design - descriptive/protocol, no abuse-related outcomes reported \\
\hline Hermoso 2006 & Ineligible study design, no abuse-related outcomes reported \\
\hline Hill 1986 & Ineligible study design - case study review \\
\hline Hodge 1998 & Ineligible study design - survey \\
\hline Holkup 2007 & Ineligible study design -community-based participatory research \\
\hline Hudson 1993 & No outcomes reported \\
\hline Hughes 1995 & Ineligible study design - post intervention survey, no abuse-related outcomes \\
\hline Hutchison 1993 & No outcomes reported \\
\hline Huynh-Hohnbaum 2008 & No abuse-related outcomes \\
\hline Hwalek 1988 & Ineligible study design - secondary data, descriptive \\
\hline Iris 1990 & Ineligible study design- descriptive \\
\hline Jackson 2012 & $\begin{array}{l}\text { Ineligible study design - in-depth interviews of adult protective services (APS) workers and substan- } \\
\text { tiated cases and statewide secondary data }\end{array}$ \\
\hline Jackson 2013 & Ineligible study design - qualitative interviews \\
\hline Jackson 2013a & Ineligible study design - qualitative interviews \\
\hline Jamieson 2004 & No outcomes reported \\
\hline Jenkens 2005 & Ineligible study design - case studies \\
\hline Jogerst 1997 & Ineligible study design-descriptive \\
\hline Jogerst 2001 & Ineligible study design - survey \\
\hline Jogerst 2003 & Ineligible study design - cross-sectional \\
\hline Jogerst 2004 & Ineligible study design - survey \\
\hline Jogerst 2008 & Ineligible study design - secondary data \\
\hline Jogerst 2008a & Ineligible study design - secondary data \\
\hline Johnson 1990 & Ineligible study design - survey \\
\hline
\end{tabular}




\begin{tabular}{|c|c|}
\hline Study & Reason for exclusion \\
\hline Jones 1988 & Ineligible study design - retrospective review \\
\hline Joubert 2003 & Ineligible study design - descriptive \\
\hline Joubert 2009 & Descriptive outcomes reported \\
\hline Kalavar 2012 & Ineligible study design - qualitative interviews \\
\hline Karp 2006 & No abuse-related outcomes \\
\hline Kassab 1999 & Intervention not aimed at elder abuse, no abuse-related outcomes \\
\hline Kasunic 2010 & No outcomes reported \\
\hline Keller 1996 & Post data reported \\
\hline Keswani 1997 & Ineligible study design - descriptive research approach \\
\hline Kim 2010 & Ineligible study design - post intervention \\
\hline Kiosses 2010 & No abuse-related outcomes \\
\hline Kono 2009 & No abuse-related outcomes \\
\hline Kue 2009 & Ineligible study design - retrospective case series \\
\hline Kurrle 1993 & Ineligible study design - follow-up for a year and retrospective review of cases after two years \\
\hline Kurrle 1997 & $\begin{array}{l}\text { Ineligible study design - descriptive study, with victims identified retrospectively from medical } \\
\text { records for the first three months, and } \\
\text { prospectively for remaining nine months }\end{array}$ \\
\hline Laditka 2002 & Ineligible study design - qualitative interviews \\
\hline Lantz 1997 & No abuse-related outcomes \\
\hline Lithwick 2000 & Ineligible study design - case studies \\
\hline Lori 2011 & Ineligible study design - case review and informants' interviews \\
\hline López 2008 & No abuse-related outcomes \\
\hline Malks 2002 & Aggregate outcomes \\
\hline Malks 2010 & Ineligible study design - case study \\
\hline Manthorpe 2013 & Ineligible study design - qualitative interviews \\
\hline Mariam 2015 & $\begin{array}{l}\text { Ineligible study design, not a comparison of intervention approaches, but rather a tailoring of inter- } \\
\text { ventions }\end{array}$ \\
\hline Martin-Carrasco 2009 & No abuse-related outcomes \\
\hline Mason 2003 & No outcomes reported \\
\hline
\end{tabular}




\begin{tabular}{|c|c|}
\hline Study & Reason for exclusion \\
\hline Mason 2007 & No abuse-related outcomes \\
\hline McGarry 2007 & No outcomes reported \\
\hline McGarry 2009 & No evaluation reported \\
\hline Mills 2012 & No pre and post outcomes reported \\
\hline Morris 2010 & No evaluation reported \\
\hline Murphy 2015 & Ineligible study design -pre and post intervention only, no comparison \\
\hline NAAPSA 2003 & Ineligible study design - survey \\
\hline Nakanishi 2009 & Ineligible study design - cross-sectional \\
\hline Nakanishi 2010 & Ineligible study design - post intervention evaluation, measured two time points \\
\hline Nakanishi 2013 & Ineligible study design - cross-sectional \\
\hline Nakanishi 2013a & Ineligible study design - cross-sectional \\
\hline $\begin{array}{l}\text { National Center of Elder Abuse } \\
2002\end{array}$ & Multi-component intervention, descriptive outcomes, not clear about follow-up period \\
\hline Navarro 2010 & Ineligible study design - survey \\
\hline Navarro 2013 & Ineligible study design - one-to-one propensity score matching \\
\hline Neale 1996 & Ineligible study design - secondary data, record review \\
\hline Nelson 1992 & Ineligible study design - survey \\
\hline Nerenberg 1986 & No outcomes reported \\
\hline Nusbaum 2007 & $\begin{array}{l}\text { Ineligible design, uncontrolled before-and-after study; not an interrupted time series analysis as } \\
\text { originally thought as insufficient pre-intervention measurement }\end{array}$ \\
\hline Payne 2007 & Ineligible study design \\
\hline Payne 2010 & Health aging only, no mention of abuse \\
\hline Payne 2012 & Ineligible study design \\
\hline Pellfolk 2010 & No abuse-related outcomes \\
\hline Perttu 1996 & Ineligible study design - descriptive study \\
\hline Phillips 2008 & Wrong study group - abusive elderly upon caregivers (out of scope) \\
\hline Phillips 2011 & Ineligible study design -exploratory/descriptive 2-group design \\
\hline Pillemer 1993 & $\begin{array}{l}\text { Ineligible study design - pre and post evaluation with no control, follow-up outcome measurement } \\
\text { conducted } 2 \text { months after intervention }\end{array}$ \\
\hline
\end{tabular}




\begin{tabular}{|c|c|}
\hline Study & Reason for exclusion \\
\hline Pitkala 2011 & No abuse-related outcomes \\
\hline Plotkin 1996 & No outcomes reported \\
\hline Preston-Shoot 2002 & Ineligible study design - interviews, case analysis methods and survey \\
\hline Price 1997 & Ineligible study design - interviews, case study and survey \\
\hline $\begin{array}{l}\text { Queensland Government De- } \\
\text { partment of Families } 2001\end{array}$ & No evaluation reported \\
\hline Rabinowitz 2006 & No abuse-related outcomes \\
\hline Radcliff 2013 & Ineligible study design - cross-sectional \\
\hline Reap 2002 & Ineligible study design \\
\hline Reay 2002 & $\begin{array}{l}\text { This study was originally thought to be eligible as an interrupted time series analysis. There is no } \\
\text { comparison group. The intervention is provided step-wise in } 2 \text { stages like a 'care-bundle', and ac- } \\
\text { cording to EPOC guidelines all these observations are handled as one intervention and one mea- } \\
\text { sure. }\end{array}$ \\
\hline Riessman 1982 & Ineligible study design - survey \\
\hline Roush 2012 & No evaluation or outcomes reported \\
\hline Saltz 2007 & $\begin{array}{l}\text { Multi-themes curriculum on geriatric not specific to elder abuse, no elder abuse-related outcome } \\
\text { reported }\end{array}$ \\
\hline Sanders 2008 & No abuse-related outcome reported \\
\hline Schaffer 1999 & Ineligible study design - face-to-face interviews, focus groups, and phone-in \\
\hline Schonfeld 2006 & Ineligible study design - case control \\
\hline Scogin 1990 & No intervention, wrong study design - survey \\
\hline Scogin 1990a & No outcomes reported \\
\hline
\end{tabular}

Seamon $1996 \quad$ Ineligible study design - pre and post evaluation with no control, 2 measurements with baseline and immediate post intervention measurement

\begin{tabular}{ll}
\hline Seamon 1997 & $\begin{array}{l}\text { Ineligible study design - pre and post evaluation with no control, 2 measurements with baseline } \\
\text { and immediate post intervention } \\
\text { measurement }\end{array}$ \\
\hline Shah 2008 & $\begin{array}{l}\text { Ineligible study design - pre and post evaluation with no control, 2 measurements with baseline } \\
\text { and post } 6 \text { months measurement }\end{array}$ \\
\hline Shefet 2007 & $\begin{array}{l}\text { Multi-themes curriculum including domestic violence, not specific to elder abuse, no elder abuse- } \\
\text { related outcomes reported }\end{array}$ \\
\hline Shugarman 2003 & Ineligible study design - cross-sectional \\
\hline Signe 2008 & No abuse-related outcomes \\
\hline \hline $\begin{array}{l}\text { Interventions for preventing abuse in the elderly (Review) } \\
\text { Copyright } \odot 2016 \text { The Cochrane Collaboration. Published by John Wiley \& Sons, Ltd. }\end{array}$
\end{tabular}




\begin{tabular}{|c|c|}
\hline Study & Reason for exclusion \\
\hline Simon 1992 & Ineligible study design - interviews \\
\hline Smith 2010 & No outcomes reported \\
\hline Spijker 2011 & No abuse-related outcomes \\
\hline Spijker 2013 & No abuse-related outcomes \\
\hline Stark 2012 & Ineligible study design - editorial \\
\hline Strümpel 2011 & Ineligible study design - qualitative interviews \\
\hline Sugita 2011 & $\begin{array}{l}\text { Ineligible study design - pre and post evaluation with no control, } 2 \text { measurements with baseline } \\
\text { and immediate post measurement }\end{array}$ \\
\hline Tapper 2010 & No outcomes reported \\
\hline Teaster 2003 & Ineligible study design - survey \\
\hline Teaster 2003a & Ineligible study design - survey \\
\hline Teaster 2004 & Ineligible study design - aggregated data from adult protective services (APS) case files \\
\hline Teaster 2004a & Ineligible study design - secondary data, cumulative frequency of outcomes reported \\
\hline Teaster 2006 & Ineligible study design - survey \\
\hline Teaster 2010 & Ineligible study design - survey \\
\hline Teitelman 2000 & No outcomes reported \\
\hline Testad 2005 & No abuse-related outcomes \\
\hline Tompkins 2009 & No abuse-related outcomes \\
\hline Uva 1996 & $\begin{array}{l}\text { Ineligible study design - pre and post evaluation with no control, with baseline and post measure- } \\
\text { ment }\end{array}$ \\
\hline Vaccaro 1990 & Ineligible study design pre and post intervention with no control, small sample size = 6 \\
\hline Vaccaro 1992 & Ineligible study design, no outcomes on elder abuse, small size sample \\
\hline $\begin{array}{l}\text { Victoria Department for Victo- } \\
\text { rian Communities } 2006\end{array}$ & No evaluation or outcomes reported \\
\hline Vinton 1993 & Ineligible study design - pre and post evaluation with baseline and immediate measurements \\
\hline Vinton 1997 & Ineligible study design - survey \\
\hline Vladescu 2000 & Ineligible study design - retrospective review \\
\hline Wagenaar 2009 & Ineligible study design \\
\hline Wasylkewycz 1994 & No outcomes reported \\
\hline
\end{tabular}




\begin{tabular}{ll}
\hline Study & Reason for exclusion \\
\hline Watson 1994 & Ineligible study design - descriptive \\
\hline Weiner 1991 & Ineligible study design - post-evaluation feedback \\
\hline Wiglesworth 2006 & Ineligible study design - survey, case studies and structured interviews \\
\hline Williams 2004 & No outcomes reported \\
\hline Williams 2007 & No abuse-related outcomes \\
\hline Wolf 2000 & Ineligible study design - case review with baseline assessment and reassessment (at 6 months) \\
\hline Yan 2014 & Ineligible study design - no control \\
\hline
\end{tabular}

Characteristics of ongoing studies [ordered by study ID]

Loh 2015

\begin{tabular}{ll}
\hline Trial name or title & Improving nurses' detection and management of elder abuse and neglect (I-NEED) \\
\hline Methods & $\begin{array}{l}\text { Multi-site, three-armed, community-based cluster randomised controlled trial with 6-months fol- } \\
\text { low-up }\end{array}$ \\
\hline Participants & Community and registered nurses from government health clinics \\
\hline Interventions & $\begin{array}{l}\text { Three-phased study, premised on the Precede-Proceed Model, comprises baseline focus group dis- } \\
\text { cussion and survey (Phase 1), development of training module (Phase 2) and implementation and } \\
\text { evaluation of the training (Phase 3) }\end{array}$ \\
\hline Outcomes & $\begin{array}{l}\text { Knowledge and awareness on elder abuse and neglect and the number of cases identified and } \\
\text { managed during follow-up }\end{array}$ \\
\hline Starting date & $\begin{array}{l}\text { 2014 } \\
\text { Contact information }\end{array}$ \\
\hline Wotes & $\begin{array}{l}\text { funded by the University of Malaya Research Grant (RP001C-13HTM), (FL002-13SBS), and the Uni- } \\
\text { versity of Malaya Grand Challenge Programme: Pre- venting Elder Abuse and Neglect Initiative } \\
\text { (PEACE) (GC001C-14HTM). }\end{array}$ \\
\hline
\end{tabular}

\section{ADDITIONAL TABLES}

Table 1. Summary of main characteristics of included studies

\begin{tabular}{lllll}
\hline Study & Intervention category & Study design & $\begin{array}{l}\text { Sample } \\
\text { size }\end{array}$ & $\begin{array}{l}\text { Population of } \\
\text { Interest }\end{array}$
\end{tabular}

\section{Educational Interventions targeted at health professionals and/or carers}


Table 1. Summary of main characteristics of included studies (Continued)

\begin{tabular}{|c|c|c|c|c|c|c|}
\hline Hsieh 2009 & Educational Interventions & $\begin{array}{l}\text { Controlled be- } \\
\text { fore-and-after } \\
\text { study }\end{array}$ & $\begin{array}{l}112 \text { (re- } \\
\text { cruited) }\end{array}$ & Caregivers & Taiwan & $6 / 11$ \\
\hline $\begin{array}{l}\text { Richardson } \\
2002\end{array}$ & Educational Interventions & $\begin{array}{l}\text { Randomised con- } \\
\text { trolled trial }\end{array}$ & 86 & $\begin{array}{l}\text { Health per- } \\
\text { sonnel }\end{array}$ & $\begin{array}{l}\text { United } \\
\text { Kingdom }\end{array}$ & $8 / 11$ \\
\hline Teresi 2013 & Educational Interventions & $\begin{array}{l}\text { Cluster-randomised } \\
\text { controlled trial }\end{array}$ & 1405 & $\begin{array}{l}\text { Nursing home } \\
\text { residents }\end{array}$ & $\begin{array}{l}\text { United } \\
\text { States }\end{array}$ & $6 / 11$ \\
\hline \multicolumn{7}{|c|}{ Progammes to reduce factors influencing elder abuse } \\
\hline $\begin{array}{l}\text { Cooper } \\
2015\end{array}$ & $\begin{array}{l}\text { Programmes to reduce factors } \\
\text { influencing elder abuse }\end{array}$ & $\begin{array}{l}\text { Randomised con- } \\
\text { trolled trial }\end{array}$ & 260 & $\begin{array}{l}\text { Carers (family } \\
\text { members) }\end{array}$ & $\begin{array}{l}\text { United } \\
\text { Kingdom }\end{array}$ & $10 / 11$ \\
\hline \multicolumn{7}{|c|}{ Programmes to increase detection } \\
\hline $\begin{array}{l}\text { Bartels } \\
2005\end{array}$ & $\begin{array}{l}\text { Programmes to increase detec- } \\
\text { tion }\end{array}$ & $\begin{array}{l}\text { Controlled be- } \\
\text { fore-and-after } \\
\text { study }\end{array}$ & $\begin{array}{l}44 \text { clini- } \\
\text { cians; } 100 \\
\text { elderly peo- } \\
\text { ple }\end{array}$ & $\begin{array}{l}\text { Clinicians and } \\
\text { elderly con- } \\
\text { sumer }\end{array}$ & $\begin{array}{l}\text { United } \\
\text { States }\end{array}$ & $2 / 11$ \\
\hline
\end{tabular}

\section{Programmes targeted to victims}

\begin{tabular}{llllll}
\hline Davis 2001 & $\begin{array}{l}\text { Multi-component intervention } \\
\text { of community-wide education } \\
\text { and then individual level inter- } \\
\text { vention by police and social } \\
\text { workers }\end{array}$ & $\begin{array}{l}\text { Randomised con- } \\
\text { trolled trial }\end{array}$ & 403 & $\begin{array}{l}\text { Victims of el- } \\
\text { der abuse }\end{array}$ & $\begin{array}{l}\text { United } \\
\text { States }\end{array}$ \\
\hline $\begin{array}{l}\text { Brownell } \\
2006\end{array}$ & $\begin{array}{l}\text { Psycho-educational support } \\
\text { group structured with educa- } \\
\text { tional content }\end{array}$ & $\begin{array}{l}\text { Randomised con- } \\
\text { trolled trial }\end{array}$ & 16 & Abused elder & United \\
women & $2 / 11$ & States \\
\hline
\end{tabular}




\begin{tabular}{|c|c|c|c|c|c|c|c|c|}
\hline Study & $\begin{array}{l}\text { Communi- } \\
\text { ty Partner- } \\
\text { ships }\end{array}$ & $\begin{array}{l}\text { Level of In- } \\
\text { tervention }\end{array}$ & $\begin{array}{l}\text { Reach of } \\
\text { Strategies }\end{array}$ & $\begin{array}{l}\text { Magnitude of } \\
\text { Intervention }\end{array}$ & $\begin{array}{l}\text { Cost Per } \\
\text { Person }\end{array}$ & Intensity & Overall & Assessment \\
\hline Cooper 2015 & 2 & 2 & 0 & 2 & 0 & 0 & 6 & medium \\
\hline Hsieh 2009 & 0 & 1 & 0 & 1 & 0 & 0 & 2 & low \\
\hline Richardson 2002 & 0 & 1 & 0 & 1 & 0 & 0 & 2 & low \\
\hline Teresi 2013 & 2 & 2 & 1 & 2 & 0 & 0 & 7 & high \\
\hline Bartels 2005 & 0 & 3 & 1 & 1 & 0 & 0 & 5 & medium \\
\hline Davis 2001 & 2 & 3 & 1 & 2 & 0 & 0 & 8 & high \\
\hline Brownell 2006 & 0 & 1 & 0 & 1 & 0 & 0 & 2 & low \\
\hline
\end{tabular}

Overall assessment of intensity: high (8-10), medium (5-7), low (1-4).

The overall assessment used a subjective informed determination rather than a pre-defined algorithm. The informed assessment approach was selected as the six categories presented here are not distinct, and the sufficiency of detail varies between the studies.

\section{Table 3. Matrix of outcomes reported in the included studies}

\begin{tabular}{|c|c|c|c|c|c|c|c|}
\hline Study/outcome & Hsieh 2009 & $\begin{array}{l}\text { Richardson } \\
2002\end{array}$ & Teresi 2013 & Davis 2001 & Brownell 2006 & Cooper 2015 & Bartels 2005 \\
\hline Intervention approach & $\begin{array}{l}\text { Educational } \\
\text { intervention } \\
\text { health profes- } \\
\text { sionals }\end{array}$ & $\begin{array}{l}\text { Educational } \\
\text { intervention } \\
\text { for health pro- } \\
\text { fessionals }\end{array}$ & $\begin{array}{l}\text { Educational } \\
\text { intervention } \\
\text { for carers }\end{array}$ & $\begin{array}{l}\text { Programmes } \\
\text { targeted to } \\
\text { victims, sup- } \\
\text { port group } \\
\text { and educa- } \\
\text { tion }\end{array}$ & $\begin{array}{l}\text { Programmes target- } \\
\text { ed to victims, mul- } \\
\text { ti-component, com- } \\
\text { munity education } \\
\text { and individual in- } \\
\text { tervention by police } \\
\text { and social workers }\end{array}$ & $\begin{array}{l}\text { Programs to } \\
\text { reduce factors } \\
\text { influencing el- } \\
\text { derly abuse } \\
\text { (family mem- } \\
\text { bers) }\end{array}$ & $\begin{array}{l}\text { Programs for } \\
\text { increasing de- } \\
\text { tection for } \\
\text { preventing el- } \\
\text { derly abuse }\end{array}$ \\
\hline Intervention level & $\mathrm{S}$ & $S$ & $\mathrm{~S}$ & $\mathrm{~T}$ & $\mathrm{~T}$ & $\mathrm{P}$ & $\mathrm{T}$ \\
\hline \multicolumn{8}{|l|}{ Primary outcomes } \\
\hline Abuse occurrence & & & & - & & - & \\
\hline
\end{tabular}




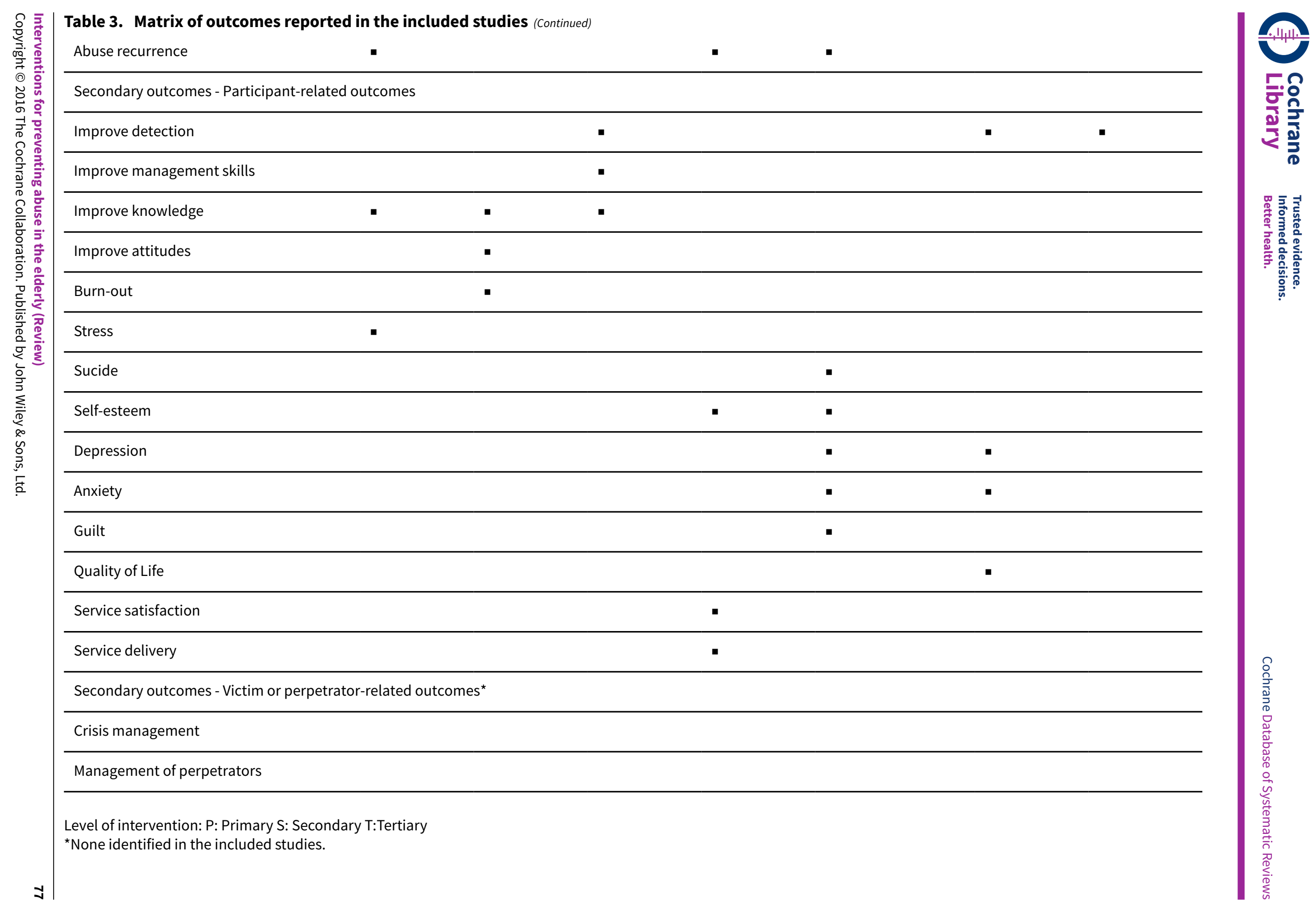


Table 4. Primary outcome - occurrence or recurrence of abuse

\begin{tabular}{|c|c|c|c|c|c|}
\hline Author & $\begin{array}{l}\text { Type of inter- } \\
\text { vention }\end{array}$ & Study design & $\begin{array}{l}\text { Type of abuse } \\
\text { measured }\end{array}$ & $\begin{array}{l}\text { Measurement } \\
\text { for outcome }\end{array}$ & Authors' FIndings \\
\hline Cooper 2015 & $\begin{array}{l}\text { Programme to } \\
\text { reduce factors } \\
\text { influencing el- } \\
\text { der abuse }\end{array}$ & $\begin{array}{l}\text { Pragmatic } \\
\text { randomised } \\
\text { parallel group } \\
\text { superiority tri- } \\
\text { al }\end{array}$ & $\begin{array}{l}\text { Occurrence of } \\
\text { abuse }\end{array}$ & $\begin{array}{l}\text { Modified con- } \\
\text { flict tactics } \\
\text { scale }\end{array}$ & $\begin{array}{l}\text { Family member carers in the intervention } \\
\text { group report lower abusive behaviour (MC- } \\
\text { TS - modified conflict tactics scale with at } \\
\text { least } 1 \text { item scoring } \geq 2 \text { ) towards the recipi- } \\
\text { ent of care compared with those in the treat- } \\
\text { ment as usual group which were not statisti- } \\
\text { cally significant } 24 \text { months. } \\
8 \text { months: } \\
\text { treatment effect: OR } 0.47,95 \% \mathrm{Cl} 0.18 \text { to } \\
1.23 \text {, P > } 0.05 \text { ( } n=214 \text { ) } \\
\text { Adjusted OR } 0.48,95 \% \mathrm{Cl} 0.18 \text { to } 1.27 \text { ( } n= \\
206 \text { ) } \\
24 \text { months: Treament effect: } \\
\text { Adjusted for baseline OR } 0.59,95 \% \mathrm{Cl} 0.27 \text { to } \\
1.28 \text { ( } n=213 \text { ) }\end{array}$ \\
\hline
\end{tabular}

\begin{tabular}{cllll}
\hline Hsieh 2009 & $\begin{array}{l}\text { Educational } \\
\text { interventions } \\
\text { (for health } \\
\text { professionals } \\
\text { and/or carers) }\end{array}$ & $\begin{array}{l}\text { Controlled be- } \\
\text { fore-and-after } \\
\text { trial }\end{array}$ & $\begin{array}{l}\text { Occurrence of } \\
\text { abuse }\end{array}$ & $\begin{array}{l}\text { Caregiver Psy- } \\
\text { chological El- } \\
\text { der Abuse Be- }\end{array}$ \\
& & & $\begin{array}{l}\text { havior Scale } \\
\text { (CPEAB) }\end{array}$
\end{tabular}

Caregivers' abusive behaviours: $(n=50$, each group analysed)

Decreased significantly after the intervention. The intervention group's change from baseline (Mean 31.22, 95\% Cl 29.53 to 32.91, SD 6.10) to post-test (Mean 29.16, $95 \% \mathrm{Cl}$ 27.49 to 30.83 , SD 6.02) $(P=0.01) .(n=100$ analysed)

No significant differences between the pre(Mean 28.98, 95\% Cl 27.36 to 30.6, SD 5.84) and post-tests (Mean $30.38,95 \% \mathrm{Cl} 28.76$ to 32 , SD 5.84) in the control group $(P<0.179)$.

Adjusted mean difference -3.46, Adjusted \% change relative to the control group $11.4 \%$ (confidence intervals can not be calculated)

Statistically significant differences between the post-test scores of the two groups relative to CPEAB ( $F=4.02, P=0.05$ and 0.02 , respectively).

\section{Brownell 2006 Programmes} for victims (Psyco-educational support groups)

$\begin{array}{ll}\text { Randomised } & \text { Recurrence } \\ \text { controlled tri- } & \text { of abuse: } \\ \text { al } & \text { "Non-phys- } \\ & \text { ical abuse"; } \\ & \text { "physical } \\ & \text { abuse" }\end{array}$

\section{Hartford}

Study Physical

Abuse Sub-

scale,

Hartford Study Nonphysical Abuse Subscale
Findings based on Intervention of 9 persons, control 6. Only women only included.

Physical abuse reported:

Pre-test: $43 \%$ of controls and $22 \%$ of intervention participants

Post-test: $0 \%$ of controls and $13 \%$ of intervention participants $(n=15$, post-intervention \& control comparison, $P=0.41$ ). Adjusted RR can not be calculated. Adjusted RD 34, $95 \% \mathrm{Cl}-23.5$ to 91.5 
Pre-test: $83 \%$ of controls and $100 \%$ of intervention participants.

Post-test: $75 \%$ of controls and $83 \%$ of intervention participants reported non-physical abuse at post-test.

Intervention \& control comparison, $\mathrm{P}=0.71$, $\mathrm{n}=15$.

Adjusted RR 0.91, $95 \mathrm{Cl} 0.58$ to 1.45 . Adjusted $\mathrm{RD}-9,95 \% \mathrm{Cl}-56.8$ to 38.8

(Neither OR or RR were provided by the authors, but calculated by review authors)

\begin{tabular}{|c|c|c|c|c|}
\hline Davis 2001 & $\begin{array}{l}\text { Programme } \\
\text { for victims } \\
\text { (including a } \\
\text { whole of com- } \\
\text { munity com- } \\
\text { ponent of } \\
\text { public educa- } \\
\text { tion) }\end{array}$ & $\begin{array}{l}\text { Nested ran- } \\
\text { domised con- } \\
\text { trolled trial }\end{array}$ & $\begin{array}{l}\text { Recurrence of } \\
\text { abuse }\end{array}$ & $\begin{array}{l}\text { Modified ver- } \\
\text { sion of Con- } \\
\text { flict Tactic } \\
\text { Scale }\end{array}$ \\
\hline
\end{tabular}

Elderly who received public education did not differ from those in the control group. However, elderly in the home visit group fared worse than those in the control group, while those who received both treatment reported more repeated victimisation.

All persons reported abuse at baseline.

Total abuse:

At 6 months, total abuse reported repeated victimisation*

No intervention: mean $5.87,95 \% \mathrm{Cl} 3.39$ to 8.35 SD 12.63

Public education: mean $3.18,95 \% \mathrm{Cl} 1.79$ to 4.57, SD 7.11,

Home visit: mean $4.61,95 \% \mathrm{Cl} 2.47$ to 6.75 , SD 10.92

Both (PE+HV): mean $12.63,95 \% \mathrm{Cl} 8.13$ to 17.13, SD 25.96.

At 12 months, total abuse reported victimisation*.

No intervention: mean $5.36,95 \% \mathrm{Cl} 3.66$ to 7.06 ,SD 8.67

Public education: mean $4.07,95 \% \mathrm{Cl} 2.32$ to 5.82, SD 8.94

Home visit: mean $3.66,95 \% \mathrm{Cl} 2.17$ to 5.15 , SD 7.62

Both (PE+HV): mean $8.58,95 \% \mathrm{Cl} 4.01$ to 13.15, SD 23.32

Hazard ratio (HR) from Cox multiple regression: Public education HR 1.26, Home visit $\mathrm{HR}=2.05$ (alpha level 0.05), Both (PE+HV) HR $=1.78$ (alpha level $=0.01), n=403$ 
Table 4. Primary outcome - occurrence or recurrence of abuse (Continued)

*The study does not specify the exact numbers of participants in each group. The review authors have made the $95 \% \mathrm{Cl}$ calculations based upon an estimate of 100 persons in each group. The $95 \% \mathrm{Cl}$ are indicative only.

Study arranged according to type of intervention, followed by study design

OR: odds ratio, RD: risk difference, RR: relative risk, SD: standard deviation

Table 5. Secondary outcomes (mixed outcomes)

$\begin{array}{llll}\text { Author } & \begin{array}{l}\text { Type of inter- Study design } \\ \text { vention }\end{array} & \begin{array}{l}\text { Types of sec- } \\ \text { ondary out- } \\ \text { comes mea- } \\ \text { sured }\end{array} & \begin{array}{l}\text { Measurement Authors' FIndings } \\ \text { for outcome }\end{array}\end{array}$

\begin{tabular}{|c|c|c|c|c|c|}
\hline Cooper 2015 & $\begin{array}{l}\text { Programme to } \\
\text { reduce factors } \\
\text { influencing el- } \\
\text { der abuse }\end{array}$ & $\begin{array}{l}\text { Pragmatic } \\
\text { randomised } \\
\text { parallel group } \\
\text { superiority tri- } \\
\text { al }\end{array}$ & $\begin{array}{l}\text { Carer-related } \\
\text { risk factors }\end{array}$ & $\begin{array}{l}\text { Hospital anx- } \\
\text { iety and de- } \\
\text { pression scale } \\
\text { health status } \\
\text { questionnaire } \\
\text { (family mem- } \\
\text { ber carers), } \\
\text { depression, } \\
\text { quality of life- } \\
\text { Alzheimer's } \\
\text { disease }\end{array}$ & $\begin{array}{l}\text { Anxiety: Mean total scores on the hospital } \\
\text { anxiety and depression scale were statisti- } \\
\text { cally lower in the intervention group than in } \\
\text { the usual care group over the eight-month } \\
\text { evaluation period: adjusted difference in } \\
\text { means }-1.80 \text { points ( } 95 \% \mathrm{Cl}-3.29 \text { to }-0.31, \mathrm{P}= \\
0.02 \text { ) and absolute difference in means }-2.0 \\
\text { points. } \\
\text { Health status (carers): Statistically higher, } \\
\text { adjusted treatment effect } 4.55 \text { ( } 95 \% \mathrm{Cl} 0.92 \\
\text { to } 8.17)(\mathrm{n}=219 \text { ) }\end{array}$ \\
\hline
\end{tabular}

Depression: Carers in the intervention group were less likely to have case-level depression (OR $0.24,95 \% \mathrm{Cl} 0.07$ to 0.76 ) and there was not a statistically significant reduction in case level anxiety $(0.30,95 \% \mathrm{Cl} 0.08$ to 1.05). Treatment effect reported adjusted for baseline score and centre: $-0.88(-1.68$ to -0.09) $(n=229)$

Quality of life: Carers' quality of life was higher in the intervention group (difference in means $4.09,95 \% \mathrm{Cl} 0.34$ to 7.83 ) but not for the recipient of care (difference in means $0.59,95 \% \mathrm{Cl}-0.72$ to 1.89$)$.

Educational interventions (for health professionals and/or carers)

$\begin{array}{ll}\text { Controlled be- } & \text { Caregivier re- } \\ \text { fore-and-af- } & \text { lated } \\ \text { ter, not ran- } & \\ \text { domised } & \end{array}$

Knowledge of Gerontology Nursing Scale (KGNS)

Work Stressors Inventory (WSI),
KGNS: Statistically significant differences were found between the post-test scores of the two groups for KGNS ( $P=0.018)$, improved significantly for the intervention group. Post-test Intervention ( $n=50$, each group)

Intervention group: Mean scores increased, pre-test mean $28.74,26.89$ to 30.59, SD 6.67 and post-intervention mean $32.96,95 \% \mathrm{Cl}$ 31.07 to 34.85 , SD 6.82 .

Control group: No significant differences between the pre- and post-tests on KGNS measures (pre-test $=$ mean $26.06,95 \% \mathrm{Cl} 23.97$ to 
Table 5. Secondary outcomes (mixed outcomes) (Continued)

28.15, SD 7.55; post-test $=$ mean 28.96, 95\%

$\mathrm{Cl} 27.17$ to 30.75 , SD $6.47 ; \mathrm{P}=0.065$ )

Adjusted mean difference 1.32 Adjusted \% change relative to control group 5\%

Stress (WSI): no effect on caregivers' perceived level of stress between intervention and control groups.

Intervention group: pre-test mean 64.14, 95\% Cl 47.53 to 61.63 , SD 20.52; post-test mean $59.42,95 \% \mathrm{Cl} 53.31$ to 65.53 , SD 22.04 .

Control group: pre-test mean $59.50,95 \% \mathrm{Cl}$ 52.37 to 66.63 , SD 25.72; post-test $=$ mean $54.58,95 \% \mathrm{CI}$ SD 25.44; $\mathrm{P}=0.330$ ).

Adjusted mean difference 3.2, adjusted \% change relative to control group $6 \%$

Overall comparison, $\mathrm{P}=0.660$.

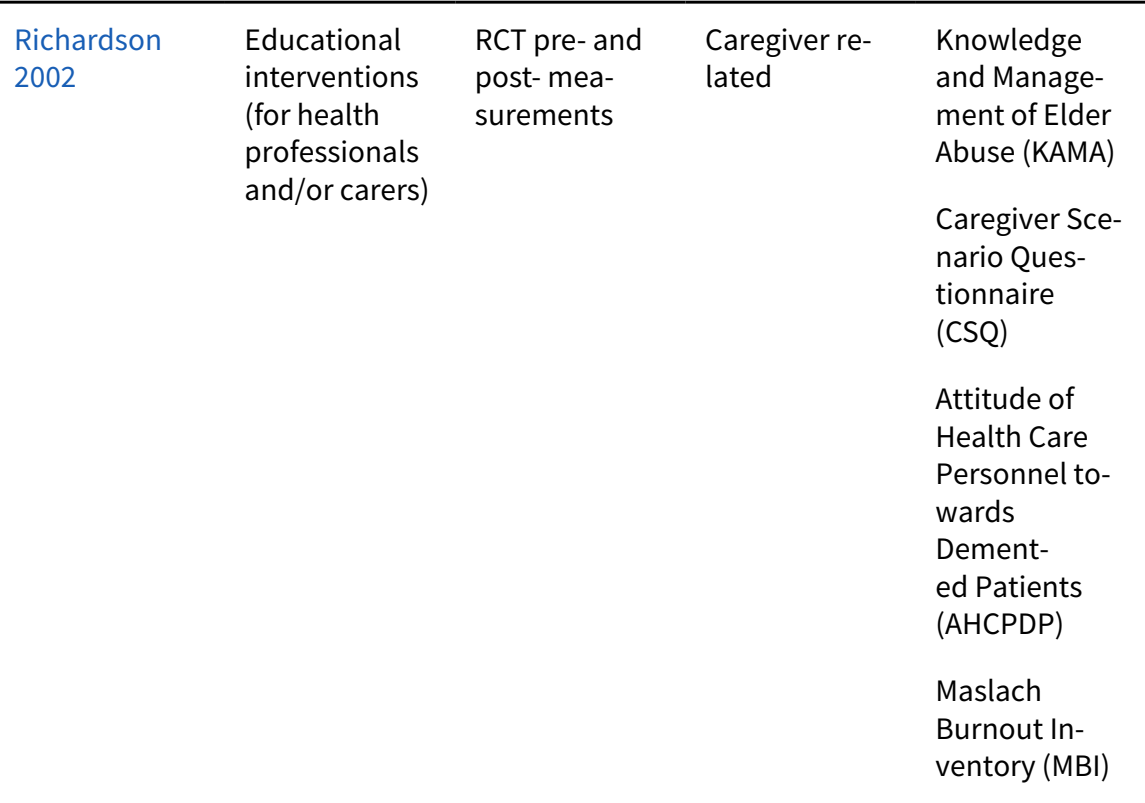

KAMA: There was a significant difference between intervention groups in final KAMA score with those randomised to Intervention ("Group 1" educational course intervention) improving after intervention and Control ("Group 2" educational material) deteriorating

Intervention mean $=3.7 ; 95 \% \mathrm{Cl} 0.85$ to 6.55 , SD 8.1,

Control mean $=-2.9 ; 95 \% \mathrm{Cl}-6.31$ to $0.51, \mathrm{SD}$ 10.0

ANOVA F=23.0; $P<0.0001)$.

Adjusted mean difference 6.6, Adjusted \% change relative to control group $25.8 \%$

Attitude: At baseline Intervention had a significantly more positive attitude than Control 2 (pre-intervention mean attitude score 13.5; SD 5.4 and 5.6; SD 4.2, respectively; $P<$ 0.0001 ; mean difference $7.9 ; 95 \% \mathrm{Cl}=5.1$ to 10.7) and post-intervention score was higher than the control Intervention: mean score $14.3,95 \% \mathrm{Cl} 12.36$ to 16.24 , SD 5.5

Control mean $6.2,95 \% \mathrm{Cl} 4.39$ to 8.01, SD 5.3

$P<0.0001$

Mean difference $8.2,95 \% \mathrm{Cl} 5.0$ to 11.2

Adjusted mean difference 0.2 , adjusted $\%$ change relative to control group $3.2 \%$

Burn-out:There were no significant differences in burn-out scores between Intervention and Control before or after intervention. 
Table 5. Secondary outcomes (mixed outcomes) (Continued)

Intervention:

Baselline $16.0,95 \% \mathrm{Cl} 12.69$ to 19.31 , SD 9.4, Follow-up $15.2 ; 95 \% \mathrm{Cl} 12.45$ to $17.95, \mathrm{SD}$

7.8.

Control Baseline $17.6,95 \% \mathrm{Cl} 13.54$ to 21.66 , SD 11.9 , Follow-up $16.7 ; 95 \% \mathrm{Cl} 12.71$ to 20.69, SD 11.7

Adjusted mean difference 0.1 , adjusted $\%$ change relative to control group $0.6 \%$

\begin{tabular}{llll}
\hline Teresi $2013 \quad \begin{array}{l}\text { Educational } \\
\text { interventions } \\
\text { (for health } \\
\text { professionals) }\end{array}$ & Cluster-RCT & $\begin{array}{l}\text { Resident-to- } \\
\text { resident el- } \\
\text { der mistreat- } \\
\text { ment focus } \\
\text { with caregiver }\end{array}$ & $\begin{array}{l}10 \text { items } \\
\text { knowledge } \\
\text { test. Resi- } \\
\text { dent-to-Res- } \\
\text { ident Elder } \\
\text { Mistreatment } \\
\text { Behavior } \\
\text { Recognition }\end{array}$ \\
& & and Docu- \\
& & mentation \\
& & Sheets (R- \\
& & REM-BRDS)
\end{tabular}

Dectection incidents in the past 2-weeks:

Intervention: $(\mathrm{n}=720)$

Baseline: $(\mathrm{n}=353)$, mean $0.51,95 \% \mathrm{Cl} 0.22$ to 0.8, SD 2.79

At 6 months: $(\mathrm{n}=580$, mean $1.08,95 \% \mathrm{Cl} 0.48$ to 1.68, SD 7.34

12 months: $(\mathrm{n}=239)$, mean $0.51,95 \% \mathrm{Cl}, 0$ to 1.02 , SD 4.01

Control: $(n=784)$

Baseline: $(\mathrm{n}=354)$ mean $0.52,95 \% \mathrm{Cl} 0.17$ to 0.87, SD 3.4

At 6 months: $(\mathrm{n}=79)$ mean $0.27,95 \% \mathrm{Cl} 0.00$ to 0.59 , SD 1.46

12 months: $(n=23)$ mean $0.10,95 \% \mathrm{Cl} 0.00$ to 0.26 , SD 0.39

At 6 months: Adjusted mean difference 0.82 Adjusted \% change relative to control group $304 \%$

At 12 months: Adjusted mean difference 0.42 Adjusted \% change relative to control group $420 \%$

The intervention group reported more incidents at 6 and 12 months than did the control group. The sum of incidents reported during the staff interview at baseline for the previous two week period was 354 for the control group and 353 for the experimental group. After training, the six-month numbers for the control and experimental groups were 79 and 580 and at 12 months 23 and 239, respectively. (Poisson model $\mathrm{P}=$ 0.0058)

Knowledge scores, reported only for intervention group:

Nursing staff's gain in knowledge was significantly higher for both 
Table 5. Secondary outcomes (mixed outcomes) (Continued)

Module 1 (Pre-test mean $7.43,95 \% \mathrm{Cl} 7.3$ to 7.56 SD $1.16 \mathrm{n}=319$; post-test Mean 8.13, $95 \% \mathrm{Cl} 7.99$ to 8.27 , SD 1.29; $\mathrm{P}<0.001$ )

Module 2 (Pre-test mean $7.40,95 \% \mathrm{Cl} 7.22$ to $7.58 \mathrm{SD} 1.54 ; \mathrm{n}=271$; post-test mean 8.38, $95 \% \mathrm{Cl} 8.2$ to $8.56, \mathrm{SD} 1.52 ; \mathrm{P}<0.001$ ) in intervention group without comparison to the control group.

Process evaluation: showed management skills increased for the intervention group: Baseline 7.43 SE 1.54, follow-up 8.38, SE1.52.

\section{Bartels 2005}

Programme to increase detection of elder abuse

Controlled be-
fore-and-after
trial, not ran-
domised

Interview and audit of clinician practices of abuse identification
There were no differences in clinician-reported baseline assessment practices for neglect and abuse between the intervention and comparison group. However, baseline comparisons of medical records found greater documentation for neglect and abuse within intervention agencies

Neglect and abuse

Clinican interview. clinicians reporting assessment of neglect and abuse

Intervention $(\mathrm{n}=26)$

Baseline: 11.5\%

Year 1 follow-up : $65.4 \%$

Control $(n=18)$

Baseline: $22.2 \%$

Year 1 follow-up 38.9\%

Reported OR $=6.50$

Authors state that at 1-year follow-up, there was a significant increase in the proportion of clinicians in the intervention group (baseline $11.5 \%$, follow-up $65.4 \%$ ) who reported routinely conducting clinical assessments in neglect and abuse domain. In contrast, there was little change in reported clinical practices by clinicians in the comparison sites (baseline 22.2\%, follow-up 38.9\%). The analyses compared pre-post change scores between the intervention group and the comparison group and have reported OR of 6.50 , however neither the $P$ value or confidence interval is provided to substantiate the claim that it is 'significant'. $(n=44)$

Re-analysis by review authors: adjusted RD $37.2,95 \% \mathrm{Cl}-3.5$ to 77.9 ; adjusted RR 3.24, $95 \% \mathrm{Cl} 0.75$ to 13.9 (not statistically different) 
Table 5. Secondary outcomes (mixed outcomes) (Continued)

Chart audit: The authors states that there was a significant increase in the proportion of charts which documented neglect and abuse in the intervention group (baseline $19.7 \%$; follow-up $91.8 \%$ ) compared to the comparison group (baseline $0 \%$, follow-up $2.6 \%)$ at follow-up.

Chart audit:

Intervention ( $\mathrm{n}=61$ charts audited)

Baseline $19.7 \%$

1-year follow-up: $91.8 \%$

Control $(n=39)$

Baseline 0.0\%

Follow-up 2.6\%

'Odds ratio could not be calculated'

Re-analysis by review authors: adjusted RD $69.5,95 \% \mathrm{Cl} 62.9$ to 71.1 ; adjusted RR could not be calculated.

\section{Brownell 2006 Programme} for victims Randomised controlled tri(psycho-edual

Victims: sense
of control, so-
cial support,
alcohol abuse,
depression,
drug use, fam-
ily relation-
ship prob-
lems, guilt,
suicide, anxi-
ety and soma-
tisation
port groups)

CESB-D 10
Hartford
Study;
Guilt Sub-
scale;
Rosenberg
Self-Esteem
Scale;
Health Lo-
cus of Control
Scale;

Medical Outcomes

Study Social Support Survey;

BSI-18
Recruitment of 16 women, 15 completers. 9 intervention, 6 control

There were no significant changes in outcome measures for either control or intervention group participants after the intervention ended; depression, guilt, and selfesteem $(n=15)$.

Depression: $14 \%$ of controls and $56 \%$ of intervention participants suffered from depression at pre-test. $33 \%$ of controls and $56 \%$ of intervention participants suffered from depression at post-test. (Post, intervention \& control comparison $\mathrm{P}=0.49$ ).

Guilt: $28 \%$ of the control participants scored above threshold and $33 \%$ of the intervention participants scored above threshold at pre-test. $14 \%$ of the control participants scored above threshold and $22 \%$ of the intervention participants scored above threshold at post-test.(post, intervention \& control comparison $\mathrm{P}=0.75)$. Calculated adjusted RR 1.33 (favouring control) $95 \% \mathrm{Cl} 0.30$ to 5.89. Adjusted RD 3, $95 \mathrm{Cl}-11.5$ to 17.5 .

Self-Esteem (Rosenberg scale): Authors stated "Participants scored an average of 32, which is above the midpoint." Average score for either groups not reported.

Findings on Sense of Control and Social Support, anxiety and somatisation not reported. 
Table 5. Secondary outcomes (mixed outcomes) (Continued)

\begin{tabular}{|c|c|c|c|c|c|}
\hline Davis 2001 & $\begin{array}{l}\text { Programme } \\
\text { for victims } \\
\text { (including a } \\
\text { whole of com- } \\
\text { munity com- } \\
\text { ponent of } \\
\text { public educa- } \\
\text { tion) }\end{array}$ & $\begin{array}{l}\text { Nested ran- } \\
\text { domised con- } \\
\text { trolled trial }\end{array}$ & $\begin{array}{l}\text { Victims: } \\
\text { i) knowledge } \\
\text { of services } \\
\text { ii) satisfaction } \\
\text { with police } \\
\text { iii) assess- } \\
\text { ment of ser- } \\
\text { vice delivery } \\
\text { iv) self-esteem } \\
\text { v) well-being } \\
\text { of victims }\end{array}$ & $\begin{array}{l}\text { i) six-item Use } \\
\text { of Services } \\
\text { Scale } \\
\text { ii) self-devel- } \\
\text { oped ques- } \\
\text { tions } \\
\text { iii) self-devel- } \\
\text { oped ques- } \\
\text { tions } \\
\text { iv) Rosenberg } \\
\text { Self-esteem } \\
\text { v) Bradburn } \\
\text { Affect-Balance } \\
\text { Scale }\end{array}$ & $\begin{array}{l}\text { No baseline data are provided for compari- } \\
\text { son, The study authors stated there was no } \\
\text { significant difference between intervention } \\
\text { and control group in relation to knowledge } \\
\text { about elder abuse or awareness and use of } \\
\text { services at 6th or } 12 \text { th months. There is no } \\
\text { significant difference between experimen- } \\
\text { tal and control group in their psychological } \\
\text { states. } \\
\text { Without baseline comparison, no further re- } \\
\text { porting is warranted.See Davis } 2001 \text { for fur- } \\
\text { ther detail. }\end{array}$ \\
\hline
\end{tabular}

$\mathrm{Cl}$ : confidence interval, OR: odds ratio, RD: risk difference, RR: relative risk, SD: standard deviation

\section{Appendix 1. Bibliographic database searches}

\begin{tabular}{|c|c|c|c|}
\hline Database & Platform & Day Search & Hits \\
\hline AgeLine & EBSCO & 17 September 2015 & 2938 \\
\hline CINAHL & EBSCO & 7 March 2016 & 1005 \\
\hline PsycINFO & EBSCO & 7 March 2016 & 978 \\
\hline MEDLINE & OVIDsp & 30 August 2015 & 11,148 \\
\hline Embase & Embase & 31 August 2015 & 9276 \\
\hline $\begin{array}{l}\text { Proquest Central, Social Services Abstracts', ASSIA, So- } \\
\text { ciological Abstracts, Dissertations and Theses Global } \\
\text { (combined) }\end{array}$ & ProQuest & 16 March 2016 & 919 \\
\hline $\begin{array}{l}\text { Web of Science (Indexes=SCI-EXPANDED, SSCI, A\&HCI, } \\
\text { CPCI-S, CPCI-SSH, BKCl-S, BKCI-SSH, CCR-EXPANDED, } \\
\text { IC) }\end{array}$ & Web of Science & 8 March 2016 & 666 \\
\hline LILACS & LILACS & 18 September 2015 & 133 \\
\hline EPPI & EPPI & 18 September 2015 & 2 \\
\hline InfoBase & InfoBase & 18 September 2015 & 2 \\
\hline $\begin{array}{l}\text { Cochrane Central Register of Controlled Trials (CEN- } \\
\text { TRAL) }\end{array}$ & CENTRAL & 18 September 2015 & 17 \\
\hline
\end{tabular}


(Continued)

Grey Literature

HMIC, Opengrey,

18 September 2015

Zetoc

AgeLine. Searched 17 September 2015

Search Strategy:

\begin{tabular}{lll}
\hline No. & Search & Results \\
\hline & ELDERLY KEYWORDS \\
\hline S1 & "aging" OR "aged" OR "senior" OR "old" OR "geriatric" OR "elder" OR "old & 6886 \\
& age" OR "pensioner" OR "veteran") AND TS=( "nursing home" OR "retirement \\
& home" OR care OR "home nurs" OR "respite care" OR emergency OR shelter \\
& OR crisis OR hospice OR "long term care" \\
& ABUSE KEYWORDS
\end{tabular}

\section{ABUSE KEYWORDS}

((harm ${ }^{\star}$ OR $_{\text {abus }}^{\star}$ ) NEAR (mental ${ }^{\star}$ OR physical ${ }^{\star}$ OR emotional ${ }^{\star}$ OR financial ${ }^{\star}$

OR sexual ${ }^{\star}$ OR psychological ${ }^{\star}$ OR material ${ }^{\star}$ OR elder $\left.{ }^{\star}\right)$ ) OR (neglect ${ }^{\star}$ OR "ill treat*" OR maltreat* OR mistreat ${ }^{\star}$ OR exploit* OR fraud* OR assault* OR crim* OR violen* OR bully* OR intimidat* OR aggress ${ }^{\star}$ OR coerc ${ }^{\star}$ OR extort ${ }^{\star}$ OR stigmati $^{\star}$ OR ostraci* OR fraud OR homicid* OR "sex offenc ${ }^{\star}$ " OR rape OR theft)

\section{APPLICATION OF THE INTERVENTION KEYWORDS}

(safeguard ${ }^{\star}$ or safe guard ${ }^{\star}$ or Prevent ${ }^{\star}$ or promot* ${ }^{\star}$ or reduc ${ }^{\star}$ or protect ${ }^{\star}$ or assist*) OR (Legislat* or Mandatory report* or advoca* or Mass media or campaign ${ }^{\star} 1$ or social marketing or increas ${ }^{\star}$ aid ${ }^{\star}$ or citizens advice) OR ( mandatory reporting/ or legislation/ or mandatory program/ or Social Marketing/ or Consumer Advocacy/ or Patient Advocacy/ or patient rights/ or mass media/ ) OR (Financial management/ or financial support/ or financing, personal/ or pensions/ or Education, Public Health Professional/ or education/ or education, nonprofessional/ or inservice training/ ) OR ( health promotion/ or health education/ or Education, Public Health Professional/) OR (Restraint reduc*) OR (intergenerational relation/ or social support/ or home care services/ or health services for the aged/) OR (Home care service* or home nursing or respite care or domiciliary care or social network ) OR ("Aged friendly cities") OR (Detect* or Screen* or home visit* or house call* or guideline* or protocol* 1 or helpline ${ }^{\star}$ or helpline* or hot-line* or hotline ${ }^{\star}$ ) OR (House call/ or Guideline Adherence/ or guideline/ or geriatric assessment/ ) OR (Crisis/ or social welfare/ or Rehabilitation/ or Transportation/ or Housing for the Elderly/ or health Policy/ or Policy/ or Organizational Policy/ or Environmental Policy/ or Social Control Policies/ or Pensions/ or social control, formal/ or hospice care/ or home care services/) OR (Emergency shelter ${ }^{\star}$ or temporary residential service ${ }^{\star}$ ) OR (emergency shelter/ or Emergencies/ or Early Medical Intervention/ or Crisis management/ ) OR ("Early Intervention (Education)"/) ) OR TS = (((elder* or older or patient ${ }^{\star}$ or continuing or public or Provider ${ }^{\star}$ or staff or service* or car$\mathrm{er}^{\star}$ or caregiver $^{\star}$ or employee $^{\star}$ or worker ${ }^{\star}$ or professional ${ }^{\star}$ or resident ${ }^{\star} 1$ or increas $^{\star}$ or money or monetary or banking or pension or legal or fraud) NEAR/5 (Education or training)) OR ((increas* or money or monetary or banking or pension or legal or fraud or cash) NEAR/3 (inform* or rights or educat* or train* or awareness or assist*)) OR (information NEAR/1 (prov* or intervention)) OR ((continuing or provider ${ }^{\star}$ or staff or service ${ }^{\star}$ or employee ${ }^{\star}$ or worker $^{\star}$ or professional ${ }^{\star}$ or increas* ${ }^{\star}$ or money or monetary or banking or pension) NEAR/5 (development or manage $\left.{ }^{\star}\right)$ OR ((care or service ${ }^{\star}$ or provider ${ }^{\star}$ or residential or 
home or institution*) NEAR/2 (policy or policies))) OR TS = (((elder* or Intergeneration $^{\star}$ or housing or transport or financ ${ }^{\star}$ or bank ${ }^{\star}$ or rehabilitation) NEAR/3 (program $^{\star}$ or project $\left.)^{\star}\right)$ OR ((social or self-help or psychological or welfare or companion*) NEAR/2 (support or system* or group* or program* or project)) OR (support NEAR/2 (system* or group* or program* or project)) OR ((neglect* or ill-treat* or maltreat ${ }^{\star}$ or mistreat ${ }^{\star}$ or exploit ${ }^{\star}$ or fraud ${ }^{\star}$ or assault ${ }^{\star}$ or crime $^{\star}$ or violen ${ }^{\star}$ or bully* or increase ${ }^{\star}$ or aggressi* or coerc $^{\star}$ or extort ${ }^{\star}$ or stigmati ${ }^{\star}$ or ostraci* $^{\star}$ or abus $\left.{ }^{\star}\right)$ NEAR/3 (recogni* or assess ${ }^{\star}$ or report $\left.\left.\left.{ }^{\star}\right)\right)\right)$ OR TS $=((($ abused or victim ${ }^{\star}$ or abuser* or perpertrator ${ }^{\star}$ or crim* or offend") NEAR/5 ("psychological" or "behavio* " or "support" or counsel* or rehabil* or support*)) OR ((Welfare OR housing or transport or banking or pension or employment or education) NEAR/3 (increas* or assist* or promot $^{\star}$ or increase* or aid))

\section{INTERVENTIONS AND ANIMAL FILTER}

"randomized controlled trial" OR "controlled clinical trial" OR "comparative study" OR intervention studies/OR evaluation studies/ OR program evaluation/OR random allocation/ OR clinical trial/ OR single-blind method/OR double-blind method/ OR control groups/OR randomized OR randomised OR placebo OR randomly OR groups OR trial OR quasi-experiment* OR pre test OR pretest OR pre-intervention OR post-intervention OR posttest OR post test OR controlled before OR "before and after stud ${ }^{*}$ OR follow-up-assessment OR (time NEAR series) OR ((evaluat* OR intervention OR interventional OR treatment) AND (control OR controlled OR study OR program* OR comparison OR "before and after" OR comparative)) OR ((intervention OR interventional OR process OR program) NEAR (evaluat* OR effect* OR outcome*)) OR Program OR programme OR secondary analys* NOT ("animal studies")

\section{ELDERLY AND ABUSE AND INTERVENTION}

The reviewers recognise this search is not optimal, but could not be updated due to unavailability of the database.

CINAHL. Searched 7 March 2016

Search Strategy:

\begin{tabular}{|c|c|c|}
\hline No & Search CINAHL with Full Text 7 March 2016 (inception 1985-2016) & \\
\hline & ELDERLY KEYWORDS & \\
\hline \multirow[t]{2}{*}{ S1 } & $\begin{array}{l}\text { (Geriatric* or Elder* or old-age or pensioner }{ }^{\star} \text { ) or ((Aging or aged or senior or } \\
\text { old }) \text { N2 (wom\#n or m\#n or lady or ladies or adult* or citizen* or resident or } \\
\text { residents or population* or people or person)) or ("Retirement home*" or "re- } \\
\text { tirement care home*") or MH "Geriatrics" }\end{array}$ & 164,139 \\
\hline & ABUSE KEYWORDS & \\
\hline S2 & 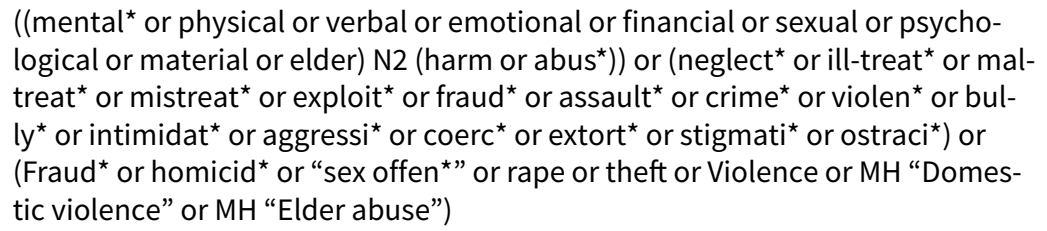 & 84,992 \\
\hline
\end{tabular}



or assist") or (Legislat" or "Mandatory report" " or advoca* or "Mass media campaign" or "social marketing" or "increas" aid" " or "citizens advice") or ("mandatory reporting" or MH "Legislation" or "mandatory program" or $\mathrm{MH}$ "Social Marketing" or MH "Patient Rights" or MH "Mass Media") or ((elder* or older or patient ${ }^{\star}$ or continuing or public or Provider" or staff or service* or car$\mathrm{er}^{\star}$ or caregiver ${ }^{\star}$ or employee ${ }^{\star}$ or worker ${ }^{\star}$ or professional ${ }^{\star}$ or resident ${ }^{\star}$ or increas* or money or monetary or banking or pension or legal or fraud) N5 (Education or training)) or ((increas* or money or monetary or banking or pension or legal or fraud or cash) N3 (inform* or rights or educat* or train* or awareness or assist $\left.{ }^{\star}\right)$ ) or (information N1 (prov ${ }^{\star}$ or intervention)) or ((continuing or provider $^{\star}$ or staff or service ${ }^{\star}$ or employee ${ }^{\star}$ or worker $^{\star}$ or professional $^{\star}$ or increas* or money or monetary or banking or pension) N5 (development or manage )) or ("Financial management" or "financial support" or financing or personal or pensions or Education or "Public Health Professional" or education or nonprofessional or "inservice training" or "health promotion" or "health education" or Education or "Public Health Service Nurses") or ((abused or victim $^{\star}$ or abuser $^{\star}$ or perpertrator ${ }^{\star}$ or crim* or offend ${ }^{\star}$ ) N5 (psychological program $^{\star}$ or behavio* therap* or social support or support group ${ }^{\star}$ or counsel ${ }^{\star}$ or rehabil $^{\star}$ or support $\left.{ }^{\star}\right)$ ) or ((care or service* or provider $^{\star}$ or residential or home or institution*) N2 (policy or policies)) or "Restraint reduc*" or ((elder* or Intergeneration ${ }^{\star}$ or housing or transport or financ ${ }^{\star}$ or bank* or rehabilitation) N3 (program* or project*)) or (MH "Support, Psychosocial" or "health services for the aged") or ("Home care service " or "home nursing" or "respite care" or "domiciliary care" or "social network") or ((social or self-help or psychological or welfare or companion*) N2 (support or system* or group* or program* or project)) or (support N2 (system* or group* or program* or project)) or "Aged friendly cities" or MH "Guideline Adherence" or (Detect* or Screen* or "home visit" " or "house call*" or guideline* or protocol* or help-line* or helpline* or hot-line or hotline $\left.^{\star}\right)$ or ((neglect* or ill-treat $^{\star}$ or maltreat ${ }^{\star}$ or mistreat* or exploit $^{\star}$ or fraud ${ }^{\star}$ or assault* or crime* or violen* or bully* or increase* or aggressi $^{\star}$ or coerc ${ }^{\star}$ or extort ${ }^{\star}$ or stigmati* or ostraci ${ }^{\star}$ or abus $\left.{ }^{\star}\right)$ N3 $\left(\right.$ recogni $^{\star}$ or assess $^{\star}$ or report $\left.{ }^{\star}\right)$ ) or ( "Guideline Adherence" or "geriatric assessment") or ((Welfare or "living standard*" or "social standing" or housing or transport or banking or pension or employment or education or "social cash" or "urban plan") N3 (increas * or assist* or promot* or increase* or aid)) or ("Crisis Intervention Services" or MH "Social Welfare+" or MH "Housing for the Elderly" or MM "Health Policy" or MM "Organizational Policies" or "Environmental Policy" or "Social Control Policies" or "social contro" or "hospice care" or "home care services") or ("Emergency shelter" or "temporary residential service"”) or (MM "Early Intervention" or "Crisis management" or "Early Intervention")

"randomi*ed controlled trial" or "controlled clinical trial" or "comparative study" or "intervention studies" or "evaluation stud"” or MM "Program Evaluation" or MH "Random Assignment" or MH "Clinical Trials" or MH "Randomized Controlled Trials" or"single-blind" or "double-blind" or "Experimental groups" or ((randomized or randomised or placebo or randomly or groups) and trial) or (time N1 series) or quasi-experiment or ("pre test" or pretest or pre-intervention or post-intervention or posttest or post test) or ("controlled before" or "before and after stud*" or "follow-up-assessment") or ((evaluat* or intervention or interventional or treatment) and (control or controlled or study or program* or comparison or "before and after" or comparative)) or ((intervention or interventional or process or program) N5 (evaluat* or effect* or outcome $\left.{ }^{\star}\right)$ ) or (program or programme or "secondary analys") 
(Continued)

PsycINFO. Searched 7 March 2016

Search Strategy:

\begin{tabular}{|c|c|c|}
\hline No & 1975 - present & \\
\hline & ELDERLY KEYWORDS & \\
\hline \multirow[t]{2}{*}{ S1 } & $\begin{array}{l}\text { (Geriatric* or Elder* or old-age or pensioner ) or ((Aging or aged or senior or } \\
\text { old } \text { ld }^{\star} \text { N2 (wom\#n or m\#n or lady or ladies or adult* or citizen* or resident or } \\
\text { residents or population* or people or person)) or ("Retirement home*" or "re- } \\
\text { tirement care home*”) or DE "Geriatrics" }\end{array}$ & 199,856 \\
\hline & ABUSE KEYWORDS & \\
\hline \multirow[t]{2}{*}{ S2 } & 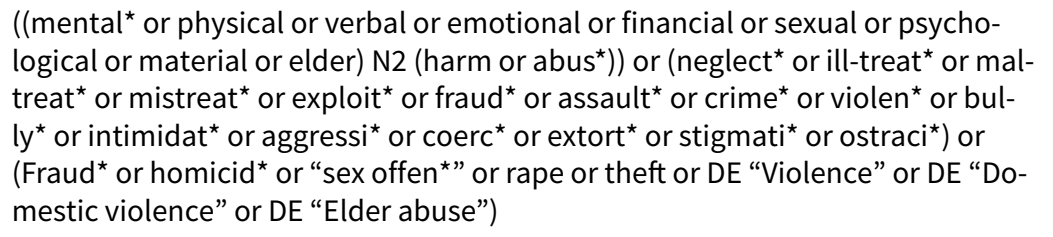 & 254,494 \\
\hline & APPLICATION OF THE INTERVENTION KEYWORDS & \\
\hline S3 & 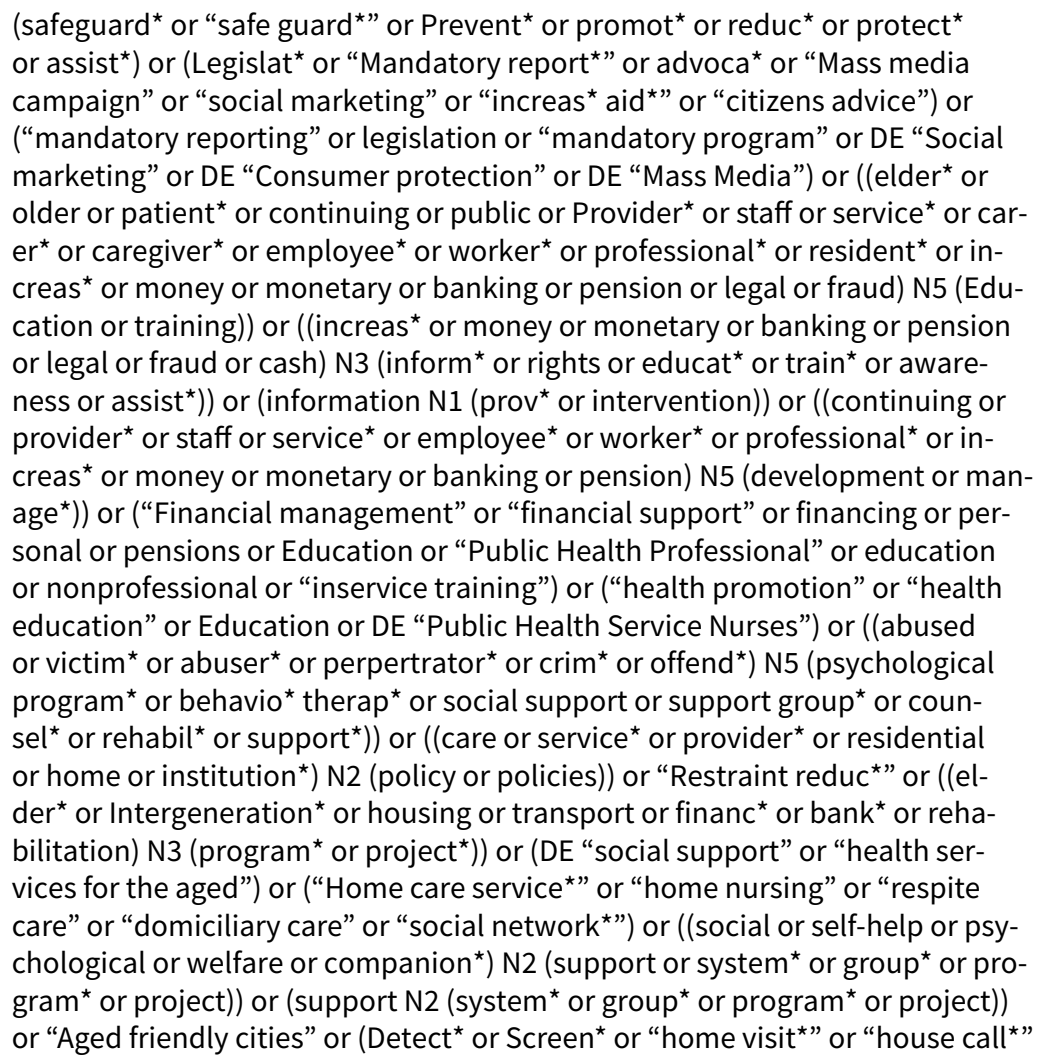 & $1,625,082$ \\
\hline
\end{tabular}


or guideline or protocol $^{\star}$ or help-line ${ }^{\star}$ or helpline* or hot-line* or hotline ${ }^{\star}$ ) or ((neglect ${ }^{\star}$ or ill-treat ${ }^{\star}$ or maltreat ${ }^{\star}$ or mistreat ${ }^{\star}$ or exploit ${ }^{\star}$ or fraud ${ }^{\star}$ or assault $^{\star}$ or crime ${ }^{\star}$ or violen ${ }^{\star}$ or bully ${ }^{\star}$ or increase ${ }^{\star}$ or aggressi ${ }^{\star}$ or coerc ${ }^{\star}$ or extort $^{\star}$ or stigmati* or ostraci* or abus $\left.{ }^{\star}\right)$ N3 $\left(\right.$ recogni ${ }^{\star}$ or assess ${ }^{\star}$ or report $\left.{ }^{\star}\right)$ ) or ( "Guideline Adherence" or DE "geriatric assessment") or ((Welfare or "living standard" or "social standing" or housing or transport or banking or pension or employment or education or "social cash" or "urban plan*") N3 (increas* or assist" or promot ${ }^{\star}$ or increase ${ }^{\star}$ or aid)) or (DE "Crisis Intervention Services" or "social welfare" or DE "Rehabilitation" or DE "Transportation" or "Housing for the Elderly" or "health Policy" or "Organizational Policy" or "Environmental Policy" or "Social Control Policies" or "social contro" or "hospice care" ) or ("Emergency shelter" or "temporary residential service" or (DE "Shelters" or

"Early Medical Intervention" or "Crisis management" or "Early Intervention")

\begin{tabular}{|c|c|c|}
\hline \multirow[t]{2}{*}{ S4 } & \multicolumn{2}{|l|}{ STUDY DESIGN KEYWORDS } \\
\hline & $\begin{array}{l}\text { "randomized controlled trial" or "controlled clinical trial" or "comparative } \\
\text { study" or "intervention studies" or "evaluation stud"” or DE "Program Evalu- } \\
\text { ation" or "random allocation" or DE "clinical trial" or "single-blind" or "dou- } \\
\text { ble-blind" or DE "Experimental groups" or ((randomized or randomised or } \\
\text { placebo or randomly or groups) and trial) or (time N1 series) or quasi-exper- } \\
\text { iment or ("pre test" or pretest or pre-intervention or post-intervention or } \\
\text { posttest or post test) or ("controlled before" or "before and after stud" or "fol- } \\
\text { low-up-assessment") or ((evaluat* or intervention or interventional or treat- } \\
\text { ment) and (control or controlled or study or program* or comparison or "be- } \\
\text { fore and after" or comparative)) or ((intervention or interventional or process } \\
\text { or program) N5 (evaluat" or effect* or outcome*)) or (program or programme } \\
\text { or "secondary analys"”) }\end{array}$ & $1,028,459$ \\
\hline S5 & S1 AND S2 AND S3 AND S4 & 2622 \\
\hline S6 & S6 Limiters - Age Groups: Aged (65 yrs \& older), Very Old ( 85 yrs \& older) & 978 \\
\hline
\end{tabular}

*Limiter: Age Groups: Aged (65 yrs \& older), Very Old ( 85 yrs \& older); Population Group: Human

\section{MEDLINE on OVID, searched 30 August 2015}

1. (Geriatric ${ }^{\star}$ or Elder ${ }^{\star}$ or old-age* or pensioner $\left.{ }^{\star}\right) . t i, a b$.

2. ((Aging or aged or senior or old ${ }^{\star}$ ) adj2 (wom\#n or m\#n or lady or ladies or adult* or citizen* or resident or residents or population*1 or people or person)).ti,ab.

3. (Retirement home* or retirement care home $\left.{ }^{\star}\right)$.ti,ab.

4. exp Aged/ or exp geriatrics/

5.1 or 2 or 3 or 4

6. ((mental ${ }^{\star}$ or physical or verbal or emotional or financial or sexual or psychological or material or elder) adj2 (harm or abus $\left.\left.{ }^{\star}\right)\right) . t i, a b$.

7. (neglect ${ }^{\star}$ or ill-treat ${ }^{\star}$ or maltreat ${ }^{\star}$ or mistreat ${ }^{\star}$ or exploit* or fraud $^{\star}$ or assault ${ }^{\star}$ or crime or violen $^{\star}$ or bully $^{\star}$ or intimidat ${ }^{\star}$ or aggressi $^{\star}$ or coerc ${ }^{\star}$ or extort ${ }^{\star}$ or stigmati* or ostraci*).ti,ab.

8. fraud/ or homicide/ or sex offenses/ or rape/ or theft/ or violence/ or domestic violence/ or elder abuse/

\section{6 or 7 or 8}

10. (safeguard* or safe guard* or Prevent $^{\star}$ or promot* or reduc $^{\star}$ or protect ${ }^{\star}$ or assist*).ti,ab.

11. (Legislat* or Mandatory report* or advoca* or Mass media or campaign*1 or social marketing or increas* aid* or citizens advice).ti,ab. 
12. mandatory reporting/ or legislation/ or mandatory program/ or Social Marketing/ or Consumer Advocacy/ or Patient Advocacy/ or patient rights/ or mass media/

13. ((elder ${ }^{\star}$ or older or patient ${ }^{\star}$ or continuing or public or Provider* or staff or service* or carer ${ }^{\star}$ or caregiver ${ }^{\star}$ or employee ${ }^{\star}$ or worker ${ }^{\star}$ or professional $^{\star}$ or resident ${ }^{\star} 1$ or increas ${ }^{\star}$ or money or monetary or banking or pension or legal or fraud) adj5 (Education or training)).ti,ab.

14. ((increas ${ }^{\star}$ or money or monetary or banking or pension or legal or fraud or cash) adj3 (inform* or rights or educat ${ }^{\star}$ or train* or awareness or assist*)).ti,ab.

15. (information adj1 (prov* or intervention)).ti,ab.

16. ((continuing or provider ${ }^{\star}$ or staff or service* or employee $^{\star}$ or worker $^{\star}$ or professional ${ }^{\star}$ or increas ${ }^{\star}$ or money or monetary or banking or pension) adj5 (development or manage*)).ti,ab.

17. Financial management/ or financial support/ or financing, personal/ or pensions/ or Education, Public Health Professional/ or education/ or education, nonprofessional/ or inservice training/

18. health promotion/ or health education/ or Education, Public Health Professional/

19. ((abused or victim ${ }^{\star}$ or abuser ${ }^{\star}$ or perpertrator ${ }^{\star}$ or crim $^{\star}$ or offend $\left.{ }^{\star}\right)$ adj5 (psychological program ${ }^{\star}$ or behavio* therap ${ }^{\star}$ or social support or support group ${ }^{\star}$ or counsel ${ }^{\star}$ or rehabil ${ }^{\star}$ or support $\left.\left.{ }^{\star}\right)\right)$.ti,ab.

20. ((care or service* or provider* or residential or home or institution*) adj2 (policy or policies)).ti,ab.

21. Restraint reduc*.ti,ab.

22. ((elder ${ }^{\star}$ or Intergeneration* ${ }^{\star}$ or housing or transport or financ ${ }^{\star}$ or bank $^{\star}$ or rehabilitation) adj3 (program or project $\left.^{\star}\right)$ ).ti,ab.

23. intergenerational relation/ or social support/ or home care services/ or health services for the aged/

24. (Home care service* or home nursing or respite care or domiciliary care or social network*).ti,ab.

25. ((social or self-help or psychological or welfare or companion $\left.{ }^{\star}\right)$ adj2 (support or system or group* or program or project)).ti,ab.

26. (support adj2 (system ${ }^{\star}$ group ${ }^{\star}$ or program or project)).ti,ab.

27. Aged friendly cities.ti,ab.

28. (Detect ${ }^{\star}$ or Screen ${ }^{\star}$ or home visit ${ }^{\star}$ or house call* or guideline ${ }^{\star}$ or protocol ${ }^{\star} 1$ or help-line or helpline $^{\star}$ or hot-line or hotline $^{\star}$ ).ti,ab.

29. ((neglect ${ }^{\star}$ or ill-treat ${ }^{\star}$ or maltreat ${ }^{\star}$ or mistreat ${ }^{\star}$ or exploit* or fraud $^{\star}$ or assault ${ }^{\star}$ or crime ${ }^{\star}$ or violen ${ }^{\star}$ or bully $^{\star}$ or increase $^{\star}$ or aggressi $^{\star}$ or coerc ${ }^{\star}$ or extort ${ }^{\star}$ or stigmati* or ostraci* or abus $\left.{ }^{\star}\right)$ adj3 (recogni* or assess ${ }^{\star}$ or report $\left.\left.{ }^{\star}\right)\right) . t i$, ab.

30. House call/ or Guideline Adherence/ or guideline/ or geriatric assessment/

31. ((Welfare or living standard* or social standing or housing or transport or banking or pension or employment or education or social cash or urban plan ${ }^{\star}$ ) adj3 (increas ${ }^{\star}$ or assist* or promot $^{\star}$ or increase ${ }^{\star}$ or aid)).ti,ab.

32. Crisis/ or social welfare/ or Rehabilitation/ or Transportation/ or Housing for the Elderly/ or health Policy/ or Policy/ or Organizational Policy/ or Environmental Policy/ or Social Control Policies/ or Pensions/or social control, formal/ or hospice care/ or home care services/

33. (Emergency shelter ${ }^{\star}$ or temporary residential service*).ti,ab.

34. emergency shelter/ or Emergencies/ or Early Medical Intervention/ or Crisis management/

35. "Early Intervention (Education)"/

36.10 or 11 or 12 or 13 or 14 or 15 or 16 or 17 or 18 or 19 or 20 or 21 or 22 or 23 or 24 or 25 or 26 or 27 or 28 or 29 or 30 or 31 or 32 or 33 or 34 or 35 37.5 and 9 and 36

38. randomized controlled trial.pt.

39. controlled clinical trial.pt.

40. comparative study.pt.

41. intervention studies/ 
42. evaluation studies/

43. program evaluation/

44. random allocation/ or clinical trial/ or single-blind method/ or double-blind method/ or control groups/

45. (randomized or randomised or placebo or randomly or groups).ab.

46. trial.ti,ab.

47. (time adj series).ab,ti.

48. quasi-experiment ${ }^{\star}$.ab,ti.

49. (pre test or pretest or pre-intervention or post-intervention or posttest or post test).ab,ti.

50. (controlled before or "before and after stud*" or follow-up-assessment).ab,ti.

51. ((evaluat* or intervention or interventional or treatment) and (control or controlled or study or program* or comparison or "before and after" or comparative)).ab,ti.

52. ((intervention or interventional or process or program) adj8 (evaluat* or effect $^{\star}$ or outcome $\left.e^{\star}\right)$ ).ab,ti.

53. (program or programme or secondary analys $\left.{ }^{\star}\right) . t i, a b$.

54.38 or 39 or 40 or 41 or 42 or 43 or 44 or 45 or 46 or 47 or 48 or 49 or 50 or 51 or 52 or 53

55. exp animals/ not humans.sh.

56.54 not 55

57.37 and 56

11,148 Results

Embase, searched 31 August 2015

Search strategy

\begin{tabular}{|c|c|c|}
\hline & Search terms & comments \\
\hline \multirow[t]{3}{*}{1.} & $\begin{array}{l}\text { geriatric*:ab,ti OR elder*:ab,ti OR 'old age':ab,ti OR 'old aged':ab,ti OR pension- } \\
\text { er*:ab,ti }\end{array}$ & $\begin{array}{l}\text { Embase applies ab- } \\
\text { stract/title filter to each } \\
\text { keyword }\end{array}$ \\
\hline & & $\begin{array}{l}\text { Embase does not allow } \\
\text { truncation in phrases }\end{array}$ \\
\hline & & $\begin{array}{l}\text { Embase removes } \\
\text { parenthesis when just } \\
\text { searching OR (for exam- } \\
\text { ple) }\end{array}$ \\
\hline 2. & $\begin{array}{l}\left.\text { ((aging OR aged OR senior OR old }{ }^{\star}\right) \text { NEAR/2 (wom?n OR m?n OR lady OR ladies } \\
\text { OR adult* OR citizen^ OR resident OR residents OR population^1 OR people OR } \\
\text { person)):ab,ti }\end{array}$ & $\begin{array}{l}\text { NEAR/n used for adja- } \\
\text { cency }\end{array}$ \\
\hline 3. & $\begin{array}{l}\text { 'retirement home':ab,ti OR 'retirement homes':ab,ti OR 'retirement care } \\
\text { home':ab,ti OR 'retirement care homes':ab,ti }\end{array}$ & \\
\hline 4. & 'aged'/exp OR 'geriatrics'/exp & \\
\hline
\end{tabular}


(Continued)

5.

6.

7.
\#1 OR \#2 OR \#3 OR \#4 ( (mental ${ }^{\star}$ OR physical OR verbal OR emotional OR financial OR sexual OR psychological OR material OR elder) NEAR/2 (harm OR abus*)):ab,ti 'ill treating':ab,ti OR maltreat*:ab,ti OR mistreat*:ab,ti OR exploit*:ab,ti OR fraud $^{\star}:$ ab,ti OR assault*:ab,ti OR crime*:ab,ti OR violen*:ab,ti OR bully*ab,ti OR intimidat*:ab,ti OR aggressi ${ }^{\star}:$ ab,ti OR coerc ${ }^{\star}:$ ab,ti OR extort*:ab,ti OR stigmati* $^{\star}$ ab,ti OR ostraci*:ab,ti

8. 'fraud'/de OR 'homicide'/de OR 'sexual crime'/de OR 'rape'/de OR 'theft'/de OR 'violence'/de OR 'domestic violence'/de OR 'elder abuse'/de

Emtree terms selected to match MeSH as close as possible (not exploded)

\begin{tabular}{|c|c|c|}
\hline 9. & \#6 OR \#7 OR \#8 & \\
\hline 10. & 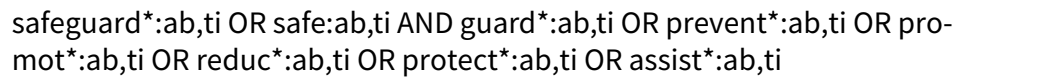 & \\
\hline 11. & $\begin{array}{l}\text { legislat*:ab,ti OR mandatory:ab,ti AND report*:ab,ti OR advoca*:ab,ti OR 'mass } \\
\text { media':ab,ti OR campaign*1:ab,ti OR 'social marketing':ab,ti OR 'increase } \\
\text { aid':ab,ti OR 'increased aid':ab,ti OR 'increasing aid':ab,ti OR 'increasingly aid- } \\
\text { ed':ab,ti OR 'citizens advice':ab,ti }\end{array}$ & \\
\hline 12. & $\begin{array}{l}\text { 'mandatory reporting'/de OR 'law'/de OR 'mandatory program'/de OR 'social } \\
\text { marketing'/de OR 'consumer advocacy'/de OR 'patient advocacy'/de OR 'pa- } \\
\text { tient right'/de OR 'mass medium'/de }\end{array}$ & \\
\hline 13. & $\begin{array}{l}\text { ((elder } \text { OR older OR patient }^{\star} \text { OR continuing OR public OR provider }{ }^{\star} \text { OR staff } \\
\text { OR service* OR carer }{ }^{\star} \text { OR caregiver }{ }^{\star} \text { OR employee* OR worker* OR profession- } \\
\text { al }^{\star} \text { OR resident }{ }^{\star} 1 \text { OR increas }{ }^{\star} \text { OR money OR monetary OR banking OR pension } \\
\text { OR legal OR fraud) NEAR/5 (education OR training)):ab,ti }\end{array}$ & \\
\hline 14. & $\begin{array}{l}\text { ((increas }{ }^{\star} \text { OR money OR monetary OR banking OR pension OR legal OR fraud } \\
\text { OR cash) NEAR/3 (inform* OR rights OR educat* OR train* OR awareness OR as- } \\
\text { sist*)):ab,ti }\end{array}$ & \\
\hline 15. & (information NEAR/1 (prov* OR intervention)):ab,ti & \\
\hline 16. & $\begin{array}{l}\text { ((continuing OR provider }{ }^{\star} \text { OR staff OR service* OR employee }{ }^{\star} \text { OR worker }{ }^{\star} \text { OR } \\
\text { professional }^{\star} \text { OR increas }{ }^{\star} \text { OR money OR monetary OR banking OR pension) } \\
\left.\left.\text { NEAR/5 (development OR manage }{ }^{\star}\right)\right): \text { ab,ti }\end{array}$ & \\
\hline 17. & $\begin{array}{l}\text { 'financial management'/de OR 'pension'/de OR 'medical education'/de OR 'ed- } \\
\text { ucation'/de OR 'in service training'/de }\end{array}$ & $\begin{array}{l}\text { Emtree: } \\
\text { financial support use: fi- } \\
\text { nancial management } \\
\text { financing, personal use: } \\
\text { financial management } \\
\text { education, public } \\
\text { health professional use: } \\
\text { medical education } \\
\text { education, nonprofes- } \\
\text { sional use: education }\end{array}$ \\
\hline
\end{tabular}


19.

((abused OR victim ${ }^{\star}$ OR abuser ${ }^{\star}$ OR perpertrator ${ }^{\star}$ OR crim OR offend ${ }^{\star}$ ) NEAR/5 ('psychological program' OR 'psychological programs' OR 'behavior therapy' OR 'behavioral therapy' OR 'behavioral therapies' OR 'behavior therapies' OR 'behaviour therapy' OR 'behavioural therapy' OR 'behavioural therapies' OR 'behaviour therapies' OR 'social support' OR 'support group' OR 'support groups' OR counsel ${ }^{*}$ OR rehabil* OR support*)):ab,ti

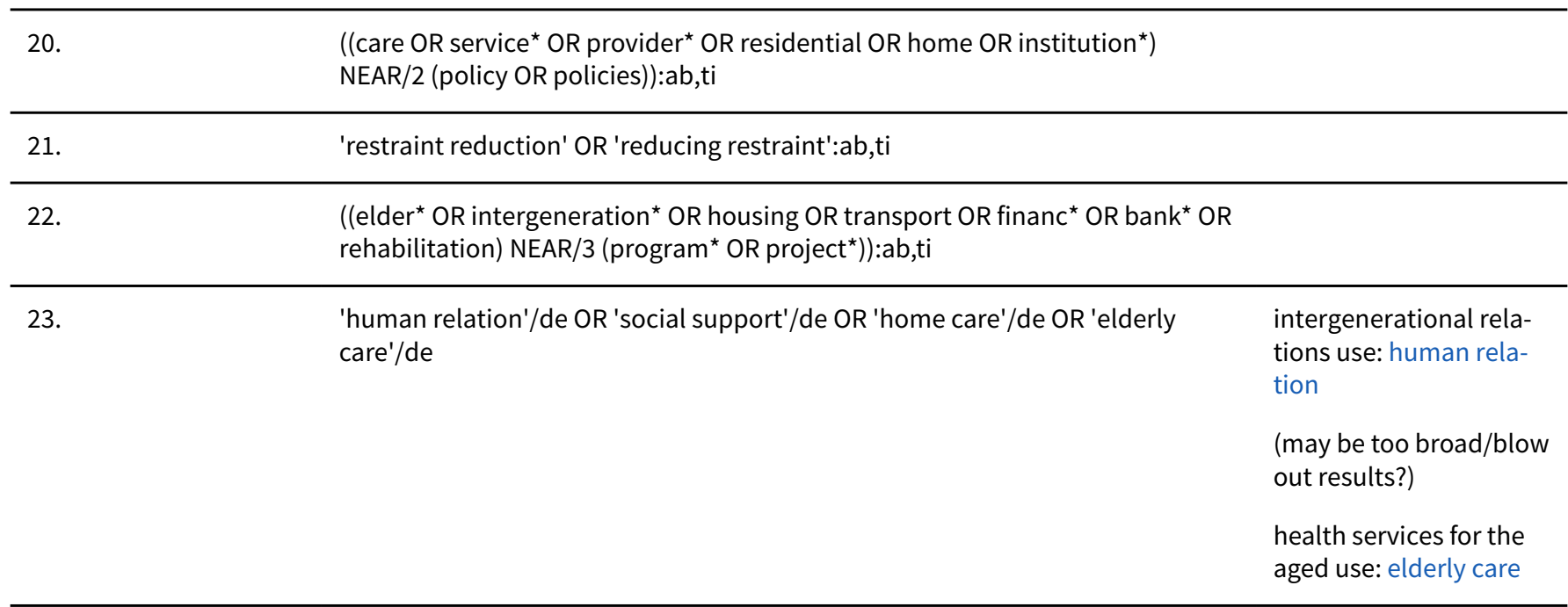

\begin{tabular}{l}
\hline 'home care service':ab,ti OR 'home care services':ab,ti OR 'home nursing':ab,ti \\
OR 'respite care':ab,ti OR 'domiciliary care':ab,ti OR 'social network':ab,ti OR \\
'social networks':ab,ti OR 'social networking':ab,ti
\end{tabular}

\section{5. ((social OR 'self help' OR psychological OR welfare OR companion $\left.{ }^{\star}\right)$ NEAR/2} (support OR system * OR group* OR program* OR project)):ab,ti

\begin{tabular}{|c|c|}
\hline 26. & (support NEAR/2 (system* OR group* OR program* OR project)):ab,ti \\
\hline 27. & 'aged friendly cities' OR 'age friendly cities':ab,ti \\
\hline 28. & $\begin{array}{l}\text { detect*:ab,ti OR screen*:ab,ti OR 'home visit':ab,ti OR 'home visits':ab,ti OR } \\
\text { 'home visiting':ab,ti OR 'house call':ab,ti OR 'house calls':ab,ti OR 'house call- } \\
\text { ing':ab,ti OR guideline*:ab,ti OR protocol*1:ab,ti OR 'help line':ab,ti OR 'help } \\
\text { lines':ab,ti OR helpline*:ab,ti OR 'hot line':ab,ti OR 'hot lines':ab,ti OR hot- } \\
\text { line*:ab,ti }\end{array}$ \\
\hline
\end{tabular}

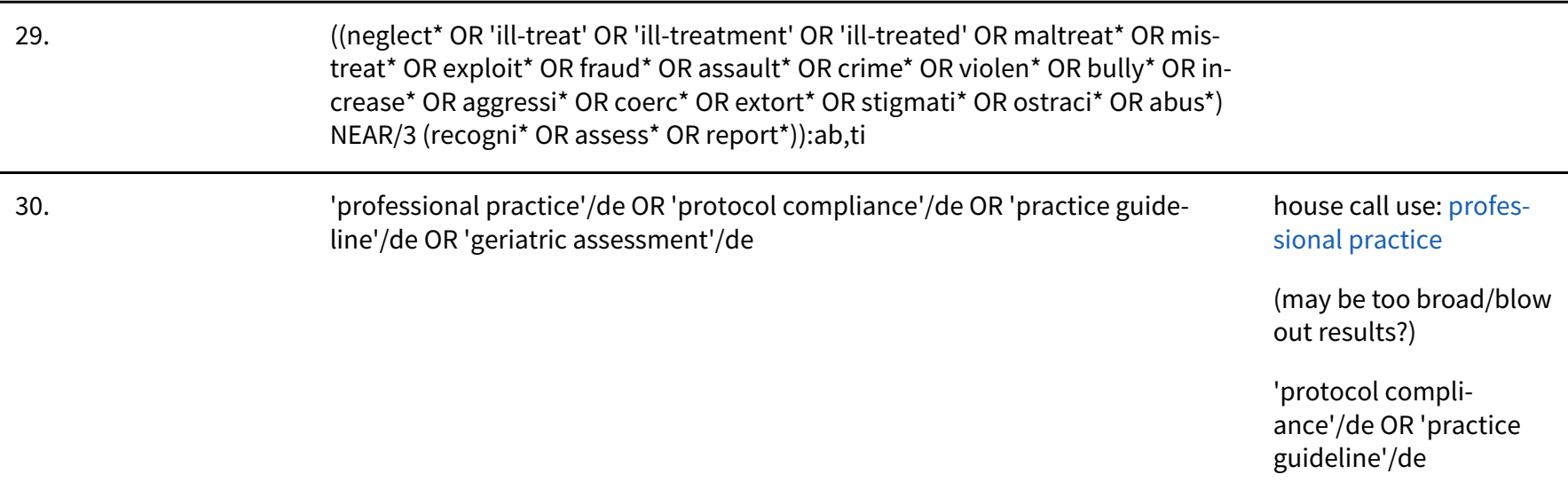


31.

((Welfare or "living standard" or "living standards" or "social standing" or housing or transport or banking or pension or employment or education or "social cash" or "urban plan" or "urban planning" or "urban planner" or "urban planners") NEAR/3 (increas* or assist* or promot* or increase* or aid)):ab,ti

32.

'crisis intervention'/de OR 'social welfare'/de OR 'rehabilitation'/de OR 'traffic and transport'/de OR 'home for the aged'/de OR 'health care policy'/de OR 'policy'/de OR 'environmental planning'/de OR 'social control'/de OR 'pension'/de OR 'hospice care'/de OR 'home care'/de
Could not find Emtree heading for 'Crisis' used 'crisis intervention'/de organizational policy use: policy

social control, formal use: social control

\section{3.} 'emergency shelter':ab,ti OR 'emergency shelters':ab,ti OR 'temporary residential service':ab,ti OR 'temporary residential services':ab,ti OR 'crisis management':ab,ti

\begin{tabular}{lll}
\hline 34. 'emergency shelter'/de OR 'emergency'/de OR 'early intervention'/de & $\begin{array}{l}\text { No Emtree equivalent } \\
\text { for Crisis management } \\
\text { MeSH found. Added as } \\
\text { phrase to previous line }\end{array}$ & $\begin{array}{l}\text { early intervention (edu- } \\
\text { cation) use: early child- } \\
\text { hood intervention }\end{array}$ \\
\hline 'early intervention'/de & & $\begin{array}{l}\text { hood } \\
\end{array}$ \\
\hline
\end{tabular}

\begin{tabular}{|c|c|c|}
\hline 36. & $\begin{array}{l}\# 10 \text { OR \#11 OR \#12 OR \#13 OR \#14 OR \#15 OR \#16 OR \#17 OR \#18 OR \#19 OR \#20 } \\
\text { OR \#21 OR \#22 OR \#23 OR \#24 OR \#25 OR \#26 OR \#27 OR \#28 OR \#29 OR \#30 OR } \\
\text { \#31 OR \#32 OR \#33 OR \#34 OR \#35 }\end{array}$ & \\
\hline 37. & \#5 AND \#9 AND \#36 & \\
\hline 38. & 'randomized controlled trial'/de & $\begin{array}{l}\text { Search by publication } \\
\text { type e.g. } \\
\text { 'randomized controlled } \\
\text { trail':it } \\
=0 \text { results } \\
\text { Emtree heading used. } \\
\text { This aligns with Study } \\
\text { Types filters. }\end{array}$ \\
\hline 39. & 'controlled clinical trial'/de & \\
\hline 40. & 'comparative study'/de & \\
\hline 41. & 'intervention study'/de & \\
\hline 42. & 'evaluation study'/de & \\
\hline 43. & 'program evaluation'/de & \\
\hline
\end{tabular}


(Continued)
44.
'randomization'/de OR 'clinical trial'/de OR 'single blind procedure'/de OR
random allocation use:
'double blind procedure'/de OR 'control group'/de
randomization

45.
s:ab

\begin{tabular}{ll}
\hline 46. & trial:ab,ti \\
\hline 47. & (time NEXT/1 series):ab,ti \\
\hline
\end{tabular}

48. 'quasi-experiment' OR 'quasi-experiments':ab,ti
49. 'pre test':ab,ti OR pretest:ab,ti OR 'pre intervention':ab,ti OR 'post interven- tion':ab,ti OR posttest:ab,ti OR 'post test':ab,ti
50. 'controlled before':ab,ti OR 'before and after study':ab,ti OR 'before and after studies':ab,ti OR 'follow-up-assessment':ab,ti

\begin{tabular}{|c|c|c|}
\hline 51. & $\begin{array}{l}\text { evaluat*:ab,ti OR intervention:ab,ti OR interventional:ab,ti OR treatment:ab,ti } \\
\text { AND (control:ab,ti OR controlled:ab,ti OR study:ab,ti OR program*:ab,ti OR } \\
\text { comparison:ab,ti OR 'before and after':ab,ti OR comparative:ab,ti) }\end{array}$ & \\
\hline 52. & $\begin{array}{l}\text { ((intervention OR interventional OR process OR program) NEAR/8 (evaluat* OR } \\
\left.\text { effect }^{\star} \text { OR outcome }{ }^{\star}\right) \text { ):ab,ti }\end{array}$ & \\
\hline 53. & $\begin{array}{l}\text { program:ab,ti OR programme:ab,ti OR 'secondary analysis':ab,ti OR 'sec- } \\
\text { ondary analyses':ab,ti }\end{array}$ & \\
\hline 54. & $\begin{array}{l}\# 38 \text { OR \#39 OR \#40 OR \#41 OR \#42 OR \#43 OR \#44 OR \#45 OR \#46 OR \#47 OR \#48 } \\
\text { OR \#49 OR \#50 OR \#51 OR \#52 OR \#53 }\end{array}$ & \\
\hline 55. & 'animal'/exp NOT 'human'/de & \\
\hline 56. & \#54 NOT \#55 & \\
\hline \multirow[t]{2}{*}{57.} & \#37 AND \#56 & $\begin{array}{l}\text { Results } 11,688 \\
\text { total }\end{array}$ \\
\hline & Limiting to source & \\
\hline 58. & \#37 AND \#56 AND [embase]/lim & $\begin{array}{l}\text { Results } \\
9276 \\
\text { Embase unique plus } \\
\text { Embase/Medline over- } \\
\text { lap }\end{array}$ \\
\hline 59. & \#58 AND [embase]/lim NOT [medline]/lim & $\begin{array}{l}\text { Results unique to Em- } \\
\text { base: } \\
2787\end{array}$ \\
\hline
\end{tabular}

ProQuest Central, Social Services Abstracts, ASSIA, Sociological Abstracts, Disertations and Thesis Global Search 16 March 2016

\section{Search strategy}

Interventions for preventing abuse in the elderly (Review) 


\begin{tabular}{|c|c|}
\hline Set & Search terms \\
\hline Elderly & Elderly Keywords \\
\hline 1. & $\begin{array}{l}\text { TI(Geriatric }{ }^{\star} \text { OR Elder }{ }^{\star} \text { OR old-age* } \text { OR pensioner }^{\star} \text { ) OR AB (Geriatric }{ }^{\star} \text { OR Elder }^{\star} \\
\left.\text { OR old-age* OR pensioner }{ }^{\star}\right)\end{array}$ \\
\hline 2. & $\begin{array}{l}\text { AB((Aging OR aged OR senior OR old*) NEAR/2 (wom?n OR m?n OR lady OR } \\
\text { ladies OR adult* OR citizen* OR resident OR residents OR population* OR peo- } \\
\text { ple OR person)) OR TI((Aging OR aged OR senior OR old*) NEAR/2 (wom?n OR } \\
\text { m?n OR lady OR ladies OR adult* OR citizen* OR resident OR residents OR pop- } \\
\text { ulation* OR people OR person)) }\end{array}$ \\
\hline 3. & $\begin{array}{l}\text { AB("Retirement home" or "retirement care home") OR TI(“Retirement } \\
\text { home*” or "retirement care home*”) }\end{array}$ \\
\hline 4. & MESH.EXACT("Aged") OR MESH.EXACT("Geriatrics") \\
\hline 5. & 1 OR 2 OR 3 OR 4 \\
\hline
\end{tabular}

\begin{tabular}{ll}
\hline Abuse & Abuse Keywords \\
\hline 6. & $\mathrm{AB}\left(\left(\right.\right.$ mental $^{*}$ or physical or verbal or emotional or financial or sexual or psy- \\
chological or material or elder) NEAR/2 (harm or abus $)) \mathrm{TI}\left(\left(\right.\right.$ mental $^{*}$ or physi- \\
cal or verbal or emotional or financial or sexual or psychological or material or \\
elder) NEAR/2 (harm or abus $))$
\end{tabular}

7. $\quad \mathrm{AB}\left(\right.$ neglect ${ }^{\star}$ or ill-treat ${ }^{\star}$ or maltreat* ${ }^{\star}$ or mistreat $^{\star}$ or exploit* or fraud $^{\star}$ or assault $^{\star}$ or crime ${ }^{\star}$ or violen ${ }^{\star}$ or bully ${ }^{\star}$ or intimidat ${ }^{\star}$ or aggressi ${ }^{\star}$ or coerc ${ }^{\star}$ or ex-

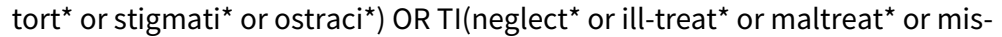
treat ${ }^{\star}$ or exploit* or fraud ${ }^{\star}$ or assault* or crime* or violen* or bully* or intimidat $^{\star}$ or aggressi* or coerc ${ }^{\star}$ or extort* or stigmati* or ostraci*)

8. MESH.EXACT("Fraud") OR MESH.EXACT("theft") or MESH.EXACT("violence") or MESH.EXACT("domestic violence") or MESH.EXACT("elder abuse")

9. 6 OR 7 OR 8

\section{Intervention Intervention Keywords}

10. $\quad$ TI(safeguard ${ }^{\star}$ or safe guard ${ }^{\star}$ or Prevent ${ }^{\star}$ or promot or reduc $^{\star}$ or protect ${ }^{\star}$ or assist $\left.^{\star}\right)$

\begin{tabular}{|c|c|}
\hline 11. & $\begin{array}{l}\text { AB("Mandatory report" or "Mass media" or "social marketing" or "increas* } \\
\text { aid"” or "citizens advice") OR TI(Legislat* or "Mandatory report"” or advoca* } \\
\text { or "Mass media" or campaign" or "social marketing" or "increas" aid" or "citi- } \\
\text { zens advice") }\end{array}$ \\
\hline
\end{tabular}
12. MESH.EXACT("mandatory reporting") or MESH.EXACT("legislation") or MESH.EXACT("mandatory program") or MESH.EXACT("Social Marketing") or MESH.EXACT("Consumer Advocacy") or MESH.EXACT("Patient Advocacy") or MESH.EXACT("patient rights") or MESH.EXACT("mass media")

13. $\mathrm{AB}\left(\left(\right.\right.$ elder* or older or patient ${ }^{\star}$ or continuing or public or Provider ${ }^{\star}$ or staff or service $^{\star}$ or carer $^{\star}$ or caregiver ${ }^{\star}$ or employee or worker $^{\star}$ or professional ${ }^{\star}$ or resident ${ }^{\star}$ or increas* or money or monetary or banking or pension or legal or fraud) NEAR/5 (Education or training)) OR TI((elder ${ }^{\star}$ or older or patient ${ }^{\star}$ or con- 
tinuing or public or Provider* or staff or service* or carer $^{\star}$ or caregiver $^{\star}$ or employee $^{\star}$ or worker ${ }^{\star}$ or professional ${ }^{\star}$ or resident ${ }^{\star}$ or increas ${ }^{*}$ or money or monetary or banking or pension or legal or fraud) NEAR/5 (Education or training))

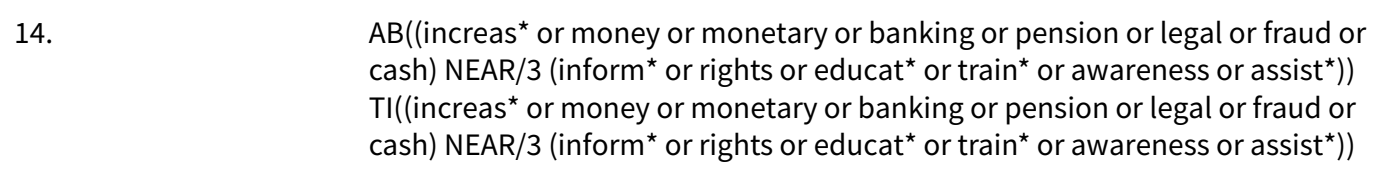

\section{5. $\mathrm{AB}$ (information NEAR/1 (prov* or intervention)) OR TI(information NEAR/1} (prov* or intervention))

\begin{tabular}{|c|c|}
\hline 16. & 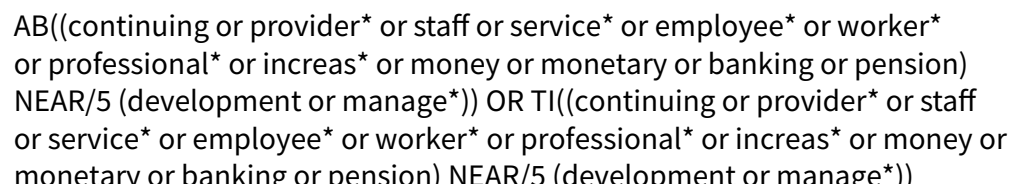 \\
\hline
\end{tabular}

MESH.EXACT("Financial management") or MESH.EXACT("financial support") or
MESH.EXACT("financing, personal") or MESH.EXACT("pensions") or MESH.EX-
ACT("Education, Public Health Professional") or MESH.EXACT("education") or
MESH.EXACT("education, nonprofessional") or MESH.EXACT("inservice train-
ing")

18. MESH.EXACT("health promotion") or MESH.EXACT("health education") or
MESH.EXACT("Education, Public Health Professional")

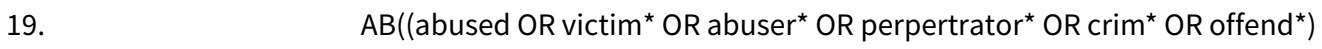 NEAR/5 ("psychological program*" OR "behavio* therap*" OR "social support" OR "support group ${ }^{\star}$ OR counsel* OR rehabil* OR support*)) OR TI((abused OR victim* OR abuser* OR perpertrator* OR crim* OR offend*) NEAR/5 ("psy- chological program*" OR "behavio* therap*" OR "social support" OR "support group ${ }^{\star}$ OR counsel* OR rehabil ${ }^{\star}$ OR support $\left.{ }^{\star}\right)$ )

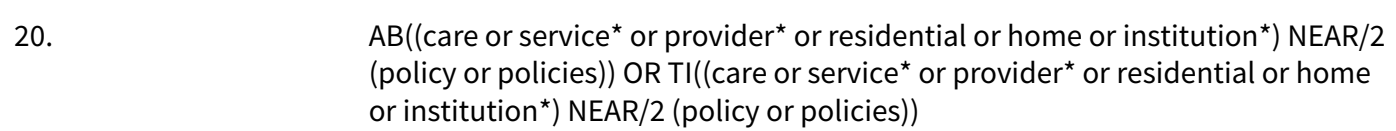

\begin{tabular}{|c|c|}
\hline 21. & $\mathrm{AB}$ (“Restraint reduc"”) OR TI("Restraint reduc*”) \\
\hline 22. & 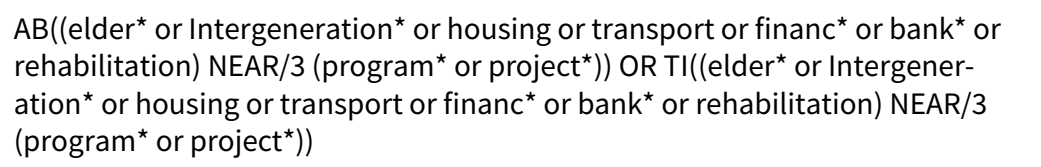 \\
\hline 23. & $\begin{array}{l}\text { MESH.EXACT("intergenerational relation") or MESH.EXACT("social support") } \\
\text { or MESH.EXACT("home care services") or MESH.EXACT("health services for the } \\
\text { aged") }\end{array}$ \\
\hline 24. & $\begin{array}{l}\text { AB("Home care service*" or "home nursing" or "respite care" or "domiciliary } \\
\text { care" or "social network*") OR TI("Home care service*" or "home nursing" or } \\
\text { "respite care" or "domiciliary care" or "social network*") }\end{array}$ \\
\hline 25. & $\begin{array}{l}\text { AB ((social or self-help or psychological or welfare or companion*) NEAR/2 } \\
\text { (support or system }{ }^{\star} \text { or group }{ }^{\star} \text { or program }{ }^{\star} \text { or project)) OR TI((social or self- } \\
\text { help or psychological or welfare or companion*) NEAR/2 (support or system } \\
\text { or group }^{\star} \text { or program }{ }^{\star} \text { or project)) }\end{array}$ \\
\hline
\end{tabular}


(Continued)

26. $A B$ (support NEAR/2 (system* or group* or program* or project)) OR TI(support NEAR/2 (system* or group* or program* or project))

\section{7.}

AB(Aged friendly cities) OR TI(Aged friendly cities)
28.

29.
TI(Detect ${ }^{\star}$ or Screen ${ }^{\star}$ or home visit ${ }^{\star}$ or house call ${ }^{\star}$ or guideline ${ }^{\star}$ or protocol $^{\star}$ or help-line ${ }^{\star}$ or helpline ${ }^{\star}$ or hot-line* or hotline ${ }^{\star}$ )

$A B\left(\left(\right.\right.$ neglect ${ }^{\star}$ or ill-treat ${ }^{\star}$ or maltreat ${ }^{\star}$ or mistreat ${ }^{\star}$ or exploit ${ }^{\star}$ or fraud ${ }^{\star}$ or assault $^{\star}$ or crime ${ }^{\star}$ or violen ${ }^{\star}$ or bully ${ }^{\star}$ or increase ${ }^{\star}$ or aggressi ${ }^{\star}$ or coerc ${ }^{\star}$ or extort $^{\star}$ or stigmati* or ostraci* or abus $\left.{ }^{\star}\right)$ NEAR/3 (recogni ${ }^{\star}$ or assess ${ }^{\star}$ or report*)) OR TI((neglect ${ }^{\star}$ or ill-treat ${ }^{\star}$ or maltreat ${ }^{\star}$ or mistreat ${ }^{\star}$ or exploit ${ }^{\star}$ or fraud ${ }^{\star}$ or assault $^{\star}$ or crime ${ }^{\star}$ or violen ${ }^{\star}$ or bully ${ }^{\star}$ or increase ${ }^{\star}$ or aggressi* or coerc ${ }^{\star}$ or extort $^{\star}$ or stigmati* or ostraci* or abus ${ }^{\star}$ ) NEAR/3 (recogni* or assess ${ }^{\star}$ or report $\left.^{\star}\right)$ )

30. MESH.EXACT("intergenerational relation") OR MESH.EXACT("social support") OR MESH.EXACT("home care services") OR MESH.EXACT("health services for the aged")

31. AB((Welfare OR "living standard*" OR social standing OR housing OR transport OR banking OR pension OR employment OR education OR social cash OR urban plan*) NEAR/3 (increas* OR assist* OR promot* OR increase* OR aid)) OR TI((Welfare OR "living standard*" OR social standing OR housing OR transport OR banking OR pension OR employment OR education OR social cash OR urban plan ${ }^{\star}$ ) NEAR/3 (increas ${ }^{\star}$ OR assist* OR promot ${ }^{\star}$ OR increase* OR aid))

32. MESH.EXACT("Crisis") or MESH.EXACT("social welfare") or MESH.EXACT("Rehabilitation") or MESH.EXACT("Transportation") or MESH.EXACT("Housing for the Elderly") or MESH.EXACT("health Policy") or MESH.EXACT("Policy") or MESH.EXACT("Organizational Policy") or MESH.EXACT("Environmental Policy") or MESH.EXACT("Social Control Policies") or MESH.EXACT("Pensions") or MESH.EXACT("social control, formal") or MESH.EXACT("hospice care") or MESH.EXACT("home care services")

\begin{tabular}{ll}
\hline 33. & $\begin{array}{l}\text { AB(“Emergency shelter" or "temporary residential service*”) OR TI("Emer- } \\
\text { gency shelter*” or "temporary residential service*”) }\end{array}$ \\
\hline 34. & $\begin{array}{l}\text { MESH.EXACT("emergency shelter") or MESH.EXACT("Emergencies") or } \\
\text { MESH.EXACT("Early Medical Intervention") or MESH.EXACT("Crisis manage- } \\
\text { ment") }\end{array}$
\end{tabular}

\begin{tabular}{ll}
\hline 35. & MESH.EXACT("Early Intervention (Education)") \\
\hline 36. & $\begin{array}{l}10 \text { or } 11 \text { or } 12 \text { or } 13 \text { or } 14 \text { or } 15 \text { or } 16 \text { or } 17 \text { or } 18 \text { or } 19 \text { or } 20 \text { or } 21 \text { or } 22 \text { or } 23 \text { or } \\
24 \text { or } 25 \text { or } 26 \text { or } 27 \text { or } 28 \text { or } 29 \text { or } 30 \text { or } 31 \text { or } 32 \text { or } 33 \text { or } 34 \text { or } 35\end{array}$ \\
\hline 37. & 5AND 9 AND 36 \\
\hline Study design & Study Design Keywords \\
\hline 38. & AB("randomized controlled trial" OR "controlled clinical trial" OR "compara- \\
& tive study") OR TI("randomized controlled trial" OR "controlled clinical trial" \\
& OR "comparative study") \\
\hline 39. & $\begin{array}{l}\text { MESH.EXACT("intervention studies") OR MESH.EXACT("intervention studies") } \\
\text { OR MESH.EXACT("program evaluation") OR MESH.EXACT("random alloca- } \\
\text { tion") or MESH.EXACT("clinical trial") or MESH.EXACT("single-blind method") } \\
\text { or MESH.EXACT("double-blind method") or MESH.EXACT("control groups") }\end{array}$ \\
\hline \hline
\end{tabular}


(Continued)

40. TI(randomized or randomised or placebo or randomly or groups)

\begin{tabular}{ll}
\hline 41. & $A B$ (trial) OR TI(trial) \\
\hline 42. & $A B$ (“time series”) OR TI(“time series”) \\
\hline 43. & $A B$ (quasi-experiment") OR TI(quasi-experiment") \\
\hline 44. & $\begin{array}{l}\text { AB("pre test" OR pretest OR pre-intervention OR post-intervention OR posttest } \\
\text { OR "post test") OR TI("pre test" OR pretest OR pre-intervention OR post-inter- } \\
\text { vention OR posttest OR post test) }\end{array}$
\end{tabular}

45.
$\mathrm{AB}\left(\left(\right.\right.$ evaluat ${ }^{\star}$ or intervention or interventional or treatment) NEAR/3 (control
or controlled or study or program ${ }^{\star}$ or comparison or "before and after" or
comparative)) OR TI((evaluat* or intervention or interventional or treatment)
NEAR/3 (control or controlled or study or program* or comparison or "before
and after" or comparative))

\begin{tabular}{|c|c|c|}
\hline 46. & 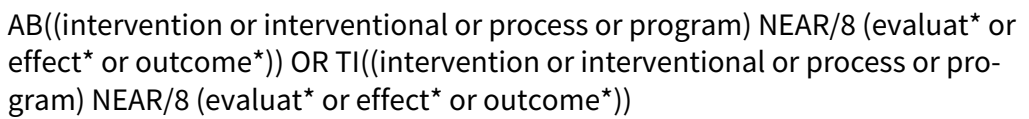 & \\
\hline 47. & $\begin{array}{l}\text { AB(program or programme or "secondary analys") OR TI(program or pro- } \\
\text { gramme or "secondary analys*”) }\end{array}$ & \\
\hline 48. & $\begin{array}{l}\text { AB("controlled before" or "before and after stud*" or "follow-up-assessment") } \\
\text { OR TI("controlled before" or "before and after stud*" or "follow-up-assess- } \\
\text { ment") }\end{array}$ & \\
\hline 49. & 38 OR 39 OR 40 or 41 or 42 or 43 or 44 or 45 or 46 or 47 or 48 & \\
\hline 50. & 37 AND 49 & \\
\hline \multirow[t]{2}{*}{51.} & Additional limits: & Results: \\
\hline & $\begin{array}{l}\text { Publication Date: After } 1975 \\
\text { Source type: Newspapers excluded }\end{array}$ & $\begin{array}{l}919 \text { (after ProQuest } \\
\text { de-duplicated final re- } \\
\text { sults) }\end{array}$ \\
\hline
\end{tabular}

Web of Science. Searched 8 March 2016

Search Strategy:

WEBOFSCCIENCE Timespan=1975-2016 (Indexes=SCI-EXPANDED, SSCI, A\&HCI, CPCI-S, CPCI-SSH, BKCI-S, BKCI-SSH, CCR-EXPANDED, IC)

\begin{tabular}{llc}
\hline Eldery & ELDERLY KEYWORDS & 88,066 \\
\hline 1 & TS= (Geriatric* or old-age* or pensioner $\left.{ }^{\star}\right)$ & 513,741 \\
\hline 2 & $\begin{array}{l}\left.\text { TS= ((Aging OR aged OR senior OR old }{ }^{\star}\right) \text { NEAR/2 (wom?n OR m?n OR lady OR } \\
\text { ladies OR adult* OR citizen* OR resident OR residents OR population* OR peo- } \\
\text { ple OR person)) }\end{array}$ & 234 \\
\hline 3 & TS= ("Retirement home*” OR “retirement care home*) \\
\hline \hline
\end{tabular}




\begin{tabular}{|c|c|}
\hline & ABUSE KEYWORDS \\
\hline 5 & $\begin{array}{l}\mathrm{TS}=\left(\left(\text { mental }{ }^{\star} \text { OR physical OR verbal OR emotional OR financial OR sexual OR }\right.\right. \\
\text { psychological OR material OR elder) NEAR/2 (harm or abus* or violence)) }\end{array}$ \\
\hline 6 & 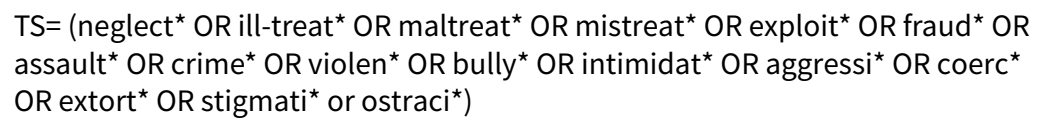 \\
\hline \multirow[t]{2}{*}{7} & \#5 OR \#6 \\
\hline & APPLICATION OF THE INTERVENTION KEYWORDS \\
\hline 8 & $\begin{array}{l}\mathrm{Tl}=\text { (safeguard* OR "safe guard"” OR Prevent* OR promot* OR reduc* OR pro- } \\
\text { tect* OR assist* OR Legislat* OR "Mandatory report*” OR advoca* OR "Mass } \\
\text { media" OR campaign OR "social marketing" OR "increas* aid"” OR "citizens } \\
\text { advice" OR "mandatory reporting" OR legislation OR "mandatory program" OR } \\
\text { "Social Marketing" OR "Consumer Advocacy" OR “Patient Advocacy" OR "Pa- } \\
\text { tient rights") }\end{array}$ \\
\hline
\end{tabular}

9 TS=((elder ${ }^{\star}$ OR older OR patient ${ }^{\star}$ OR continuing OR public OR Provider ${ }^{\star}$ OR staff OR service* OR carer ${ }^{\star}$ OR caregiver ${ }^{\star}$ OR employee ${ }^{\star}$ OR worker ${ }^{\star}$ OR professional* OR resident ${ }^{\star}$ OR increas* OR money OR monetary OR banking OR pension OR legal OR fraud) NEAR/5 (Education OR training))

\begin{tabular}{|c|c|c|}
\hline 10 & $\begin{array}{l}\text { TS }=\left(\left(\text { increas }{ }^{\star} \text { OR money OR monetary OR banking OR pension OR legal OR }\right.\right. \\
\text { fraud OR cash) NEAR/3 (inform* OR rights OR educat* OR train* OR awareness } \\
\left.\left.\text { OR assist }{ }^{\star}\right)\right)\end{array}$ & 86,411 \\
\hline 11 & $\mathrm{TS}=\left(\right.$ information NEAR/1 (prov ${ }^{\star}$ or intervention)) & 200,310 \\
\hline 12 & $\begin{array}{l}\text { TS }=\left(\left(\text { continuing OR provider }{ }^{\star} \text { OR staff or service }{ }^{\star} \text { or employee }{ }^{\star} \text { OR }_{\text {worker }}^{\star}\right.\right. \\
\text { OR professional }{ }^{\star} \text { OR increas }{ }^{\star} \text { OR money or monetary OR banking or pension) } \\
\left.\text { NEAR/5 (development OR manage })^{\star}\right)\end{array}$ & 205,155 \\
\hline 13 & $\begin{array}{l}\text { TS=("Financial management" OR "financial support" OR "financing person- } \\
\text { al" OR pensions OR "Public Health Professional" OR "inservice training" OR } \\
\text { "health promotion" OR "health education" OR "Public Health Professional Ed- } \\
\text { ucation") }\end{array}$ & 56,537 \\
\hline 14 & 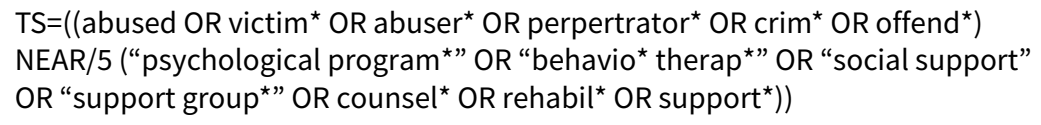 & 7,413 \\
\hline 15 & $\begin{array}{l}\text { TS }=\left(\left(\text { care OR service }{ }^{\star} \text { OR provider }{ }^{\star} \text { OR residential OR home OR institution*) }\right.\right. \\
\text { NEAR/2 (policy or policies }))\end{array}$ & 21,138 \\
\hline 16 & $\begin{array}{l}\text { TS }=\left(\left(\text { elder }{ }^{\star} \text { OR Intergeneration* OR housing or transport OR financ }{ }^{\star} \text { OR bank* }\right.\right. \\
\text { OR rehabilitation) NEAR/3 (program* OR project* }))\end{array}$ & 32,707 \\
\hline 17 & $\begin{array}{l}\text { TS=("intergenerational relation" OR "social support" OR "home care services" } \\
\text { OR "health services for the aged") }\end{array}$ & 46,306 \\
\hline 18 & $\begin{array}{l}\text { TS=("Home care service*” OR "home nursing" OR "respite care" OR "domicil- } \\
\text { iary care" OR "social network") }\end{array}$ & 22,542 \\
\hline
\end{tabular}


(Continued)
19
TS=((social OR self-help OR psychological OR welfare or companion $\left.{ }^{\star}\right)$ NEAR/2
111,293
(support OR system* OR group* OR program* OR project))

\begin{tabular}{llr}
\hline 20 & TS=(support NEAR/2 (system* OR group* OR program* OR project)) & 114,604 \\
\hline 21 & $\begin{array}{l}\text { TI=(Detect* OR Screen* OR home visit* OR “house call”* OR guideline* OR pro- } \\
\text { tocol* OR help-line* OR helpline* OR hot-line* OR hotline*) }\end{array}$ & 938,477
\end{tabular}

\begin{tabular}{|c|c|}
\hline 22 & 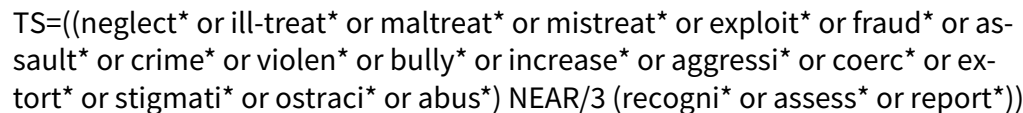 \\
\hline
\end{tabular}

\begin{tabular}{|c|c|c|}
\hline 23 & TS=(“House call” OR "Guideline Adherence" OR "geriatric assessment”) & 4765 \\
\hline 24 & $\begin{array}{l}\text { TS=((Welfare OR “living standard*” OR "social standing" OR housing OR trans- } \\
\text { port OR banking OR pension OR employment OR education OR "social cash" or } \\
\text { "urban plan"”) NEAR/3 (increas* or assist* or promot* or increase* or aid)) }\end{array}$ & 51,390 \\
\hline 25 & $\begin{array}{l}\text { TS=("social welfare" OR "Housing for the Elderly" OR "health Policy" OR "Orga- } \\
\text { nizational Policy" OR "Environmental Policy" OR "Social Control Policies" OR } \\
\text { "social control" OR "hospice care" OR "home care services") }\end{array}$ & 41,877 \\
\hline 26 & $\begin{array}{l}\text { TS=("Emergency shelter" OR "temporary residential service*” OR "Early Med- } \\
\text { ical Intervention" OR "Crisis management" OR "Early Intervention") }\end{array}$ & 18,337 \\
\hline 27 & $\begin{array}{l}\# 26 \text { OR \#25 OR \#24 OR \#23 OR \#22 OR \#21 OR \#20 OR \#19 OR \#18 OR \#17 OR \#16 } \\
\text { OR \#15 OR \#14 OR \#13 OR \#12 OR \#11 OR \#10 OR \#9 OR \#8 }\end{array}$ & $3,263,132$ \\
\hline
\end{tabular}

\section{STUDY DESIGN}

\begin{tabular}{|c|c|c|}
\hline 28 & TS=("randomized controlled trial" OR "controlled clinical trial") & 158,758 \\
\hline 29 & $\begin{array}{l}\text { TS }=((\text { comparative OR intervention or evaluation or control) NEAR/3 (studies } \\
\text { OR study OR analysis) })\end{array}$ & 665,421 \\
\hline 30 & $\begin{array}{l}\text { TS=("random allocation" OR "random assignment" OR "clinical trial" OR "sin- } \\
\text { gle-blind method" OR "double-blind method" OR "control groups") }\end{array}$ & 181,293 \\
\hline 31 & $\mathrm{TI}=($ randomized OR randomised OR placebo OR randomly) & 192,932 \\
\hline 32 & $\mathrm{TS}=$ (time NEAR/1 series) & 128,781 \\
\hline 33 & $\begin{array}{l}\text { TS=("quasi-experiment*" OR "pre test" OR pretest or pre-intervention OR post- } \\
\text { intervention OR posttest OR post test OR "controlled before" OR "before and } \\
\text { after stud" OR "follow-up-assessment") }\end{array}$ & 152,577 \\
\hline 34 & $\begin{array}{l}\mathrm{TS}=\left(\left(\text { evaluat }{ }^{\star} \text { OR intervention OR interventional OR treatment) NEAR/2 (con- }\right.\right. \\
\text { trol OR controlled OR study OR program* OR comparison OR "before and af- } \\
\text { ter" OR comparative)) }\end{array}$ & 648,431 \\
\hline 35 & $\begin{array}{l}\text { TS }=((\text { intervention OR interventional OR process OR program) NEAR/4 (evaluat* } \\
\left.\left.\text { OR effect* OR outcome }{ }^{\star}\right)\right)\end{array}$ & 378,642 \\
\hline 36 & \#35 OR \#34 OR \#33 OR \#32 OR \#31 OR \#30 OR \#29 OR \#28 & $2,092,020$ \\
\hline 37 & \#36 AND \#27 AND \#7 AND \#4 & 666 \\
\hline
\end{tabular}


LILACS. Searched 18 September 2015

Search Strategy: "Elder Abuse or Harm"[Mesh]

\section{EPPI. Searched 18 September 2015}

Search Strategy: "Elder Abuse or Harm"[Mesh]

Cochrane. Searched 18 September 2015

Search Strategy: "Elder Abuse or Harm"[Mesh]

Grey Literature. Searched 18 September 2015

Search Strategy: "Elder Abuse or Harm"[Mesh]

Grey Literature. Searched 5 July 2016

Search Strategy" "Elder abuse" "abuse of Elderly"

Appendix 2. Key organisation searches

\begin{tabular}{|c|c|}
\hline Key organisation & Hits \\
\hline Action on Elder Abuse (AEA) & 32 \\
\hline AgeConcern & 110 \\
\hline American Bars Association & 6 \\
\hline Australian Domestic \& Family Violence Clearinghouse & 101 \\
\hline Centre of Excellence on Elder Abuse \& Neglect & 31 \\
\hline Clearinghouse on Abuse and Neglect of the Elderly (CANE) & 892 \\
\hline European Network For Prevention Elder Abuse & 16 \\
\hline HelpAge International & 34 \\
\hline International Network of Agencies for Health Technology Assessment (INAHTA) & 0 \\
\hline International Labor Organization & 25 \\
\hline International Network for the Prevention of Elder abuse & 16 \\
\hline Joanna Briggs Institute EBP Database & 1 \\
\hline Joseph Rowntree Foundation & 43 \\
\hline National Adult Protective Services Association & 32 \\
\hline National Centre for the Protection of Older people & 285 \\
\hline National Centre of Caregiving & 30 \\
\hline National Clearinghouse on Abuse in Later Life & 7 \\
\hline National Committee for the Prevention of Elder Abuse & 31 \\
\hline National Guideline Clearinghouse & 2 \\
\hline
\end{tabular}


(Continued)

National Sexual Violence Resource Centre

\section{CONTRIBUTIONS OF AUTHORS}

The review was initially drafted by WYC and DF with assistance from PB, NH and SO. PB performed major revision, verification and the final editing, and is the review's contact person and lead author. Search strategies were developed by WYC, PB and DF with assistance from Ruth Turley on behalf of Cochrane Public Health. Study selection, data extraction and 'Risk of bias' assessment were conducted by WYC, $\mathrm{NH}$ and SO. WYC, NH and PB developed the GRADE and 'Summary of findings' tables; whilst DF and PB developed the grading system for assessment of intensity (Baker 2015). DF \& PB provided statistical advice and assistance with writing of the data analysis section.

\section{DECLARATIONSOF INTEREST}

Wan Yuen Choo, Noran N Hairi, Sajaratulnisah Othman, Daniel P Francis and Philip RA Baker: None to declare.

\section{SOURCES OF SUPPORT}

\section{Internal sources}

- University of Malaya Research Grant (UMRG397/12HTM), Malaysia.

- University of Malaya/Ministry of Higher Education (UM/MOHE) High Impact Research Grant E000010-20001, Malaysia.

- University of Queensland, Institute of Health and Biomedical Innovation, small project grant, Australia.

\section{External sources}

- National Health and Medical Research Council (NHMRC), Australia.

NHMRC provided funding to support the editorial process of this Cochrane Public Health review

\section{DIFFERENCES BETWEEN PROTOCOL AND REVIEW}

An earlier search in 2014 of CKNI using Chinese characters yielded no studies that were within the scope of the review. In August 2015 access to the database and the Chinese researcher was no longer available to the review authors and it was determined that obtaining access to and searching CKNI again could not be justified. Similarily, we searched AgeLine earlier, but access was unavailable in March 2016.

The information on the Ovid information guide $h t t p: / / o s p g u i d e s . o v i d . c o m / O S P g u i d e s / p r e m d b . h t m$ indicates that a search of PubMed is unnecessary as the search employed does not rely only on MESH, and the in-process articles will be identified using the strategy OVID MEDLINE downloads records from HLM daily and thus the most recent studies can be identified and in-process and alternative approaches were deemed unnecessary. MEDLINE was searched using the OVID SP MEDLINE platform and thus minimised the need for handsearching of the 10 highest yielding journals as expert information specialists consulted deemed this redundant. Bibliographic data searches originally planned, but subsequently identified as redundant because of overlap in the information sources were not run (e.g. National Health Services Database of Abstracts of Reviews of Effectiveness (DARE)).InfoBase was added. Websearches were not repeated. In an earlier approach we searched Ageline with a less optimal search, which was not repeated.

Analyses which were planned in the protocol but were not feasible or required are described in the Methods section.

The odds ratio when provided by the authors was used when the relative risk could not be calculated. For comparability across studies, we have provided calculations to adjust for baseline differences between the groups similar to Baker 2015.

We chose not to contact authors via the post as electronic means (i.e. via email) were more efficient.

As the outcomes, interventions and risk of bias varied considerably, a modified, simpler 'Summary of findings' table based on Baker 2015 was constructed. 
The original protocol stated an intent to include specific policies and legislation on elderly, however the author group subsequently felt that it would be more useful for policy makers to focus on the evaluation of evidence on policies and legislation focused on elder abuse per se. It was purposely intended to narrow the selection of studies within the large body of literature on elderly.

\section{INDEX TERMS}

\section{Medical Subject Headings (MeSH)}

Caregivers; Elder Abuse [classification] [diagnosis] [ ${ }^{*}$ prevention \& control]; Interrupted Time Series Analysis; Nursing Homes; Nursing Staff; Primary Prevention; Randomized Controlled Trials as Topic; Secondary Prevention; Tertiary Prevention

\section{MeSH check words}

Aged; Humans 